\title{
Macroscopic Effect of Quantum Gravity: Graviton, Ghost and Instanton Condensation on Horizon Scale of Universe
}

\author{
Leonid Marochnik ${ }^{1}$, Daniel Usikov ${ }^{1}$, Grigory Vereshkov ${ }^{2,3}$ \\ ${ }^{1}$ Physics Department, University of Maryland, College Park, USA \\ ${ }^{2}$ Research Institute of Physics, Southern Federal University, Rostov-on-Don, Russia, \\ ${ }^{3}$ Institute for Nuclear Research of the Russian Academy of Sciences, Moscow, Russia \\ Email: lmarochnik@gmail.com,dusikov@gmail.com,gveresh@gmail.com
}

Received June 11, 2013; revised July 12, 2013; accepted August 3, 2013

Copyright ( 2013 Leonid Marochnik et al. This is an open access article distributed under the Creative Commons Attribution License, which permits unrestricted use, distribution, and reproduction in any medium, provided the original work is properly cited.

\begin{abstract}
We discuss a special class of quantum gravity phenomena that occur on the scale of the Universe as a whole at any stage of its evolution, including the contemporary Universe. These phenomena are a direct consequence of the zero rest mass of gravitons, conformal non-invariance of the graviton field, and one-loop finiteness of quantum gravity, i.e. it is a direct consequence of first principles only. The effects are due to graviton-ghost condensates arising from the interfereence of quantum coherent states. Each of coherent states is a state of gravitons and ghosts of a wavelength of the order of the horizon scale and of different occupation numbers. The state vector of the Universe is a coherent superposition of vectors of different occupation numbers. One-loop approximation of quantum gravity is believed to be applicable to the contemporary Universe because of its remoteness from the Planck epoch. To substantiate the reliability of macroscopic quantum effects, the formalism of one-loop quantum gravity is discussed in detail. The theory is constructed as follows: Faddeev-Popov path integral in Hamilton gauge $\rightarrow$ factorization of classical and quantum variables, allowing the existence of a self-consistent system of equations for gravitons, ghosts and macroscopic geometry $\rightarrow$ transition to the one-loop approximation, taking into account that contributions of ghost fields to observables cannot be eliminated in any way. The ghost sector corresponding to the Hamilton gauge automatically ensures of one-loop finiteness of the theory off the mass shell. The Bogolyubov-Born-Green-Kirckwood-Yvon (BBGKY) chain for the spectral function of gravitons renormalized by ghosts is used to build a self-consistent theory of gravitons in the isotropic Universe. It is the first use of this technique in quantum gravity calculations. We found three exact solutions of the equations, consisting of BBGKY chain and macroscopic Einstein's equations. It was found that these solutions describe virtual graviton and ghost condensates as well as condensates of instanton fluctuations. All exact solutions, originally found by the BBGKY formalism, are reproduced at the level of exact solutions for field operators and state vectors. It was found that exact solutions correspond to various condensates with different graviton-ghost compositions. Each exact solution corresponds to a certain phase state of graviton-ghost substratum. We establish conditions under which a continuous quantum-gravity phase transitions occur between different phases of the graviton-ghost condensate.
\end{abstract}

Keywords: Quantum Gravity

\section{Introduction}

Macroscopic quantum effects are quantum phenomena that occur on a macroscopic scale. To date, there are two known macroscopic quantum effects: superfluidity at the scale of liquid helium vessel and superconductivity at the scale of superconducting circuits of electrical current. These effects have been thoroughly studied experimentally and theoretically understood. A key role in these effects is played by coherent quantum condensates of micro-objects with the De Broglie wavelength of the order of macroscopic size of the system. The third mac- roscopic quantum effect under discussion in this paper is condensation of gravitons and ghosts in the self-consistent field of the expanding Universe. A description of this effect by an adequate mathematical formalism is the problem at the present time.

We show that condensation of gravitons and ghosts is a consequence of quantum interference of states forming the coherent superposition. In this superposition, quantum fields have a certain wavelength, and with different amplitudes of probability they are in states corresponding to different occupation numbers of gravitons and ghosts. 
Intrinsic properties of the theory automatically lead to a characteristic wavelength of gravitons and ghosts in the condensate. This wavelength is always of the order of a distance to the horizon of events ${ }^{1}$.

In this fact, a common feature of macroscopic quantum effects is manifested: such effects are always formed by quantum micro-objects, whose wavelengths are of the order of macroscopic values. With this in mind, we can say that macroscopic quantum gravity effects exist across the Universe as a whole. The existence of the effects of this type was first discussed in [1].

Quantum theory of gravity is a non-renormalized theory [2] and for this reason it is impossible to calculate effects with an arbitrary accuracy in any order of the theory of perturbations. The program combining gravity with other physical interactions within the framework of supergravity or superstrings theory assumes the ultimate formulation of the theory containing no divergences. Today we do not have such a theory; nevertheless, we can hope to obtain physically meaningful results. Here are the reasons for this assumption.

First, in all discussed options for the future theory, Einstein's theory of gravity is contained as a low energy limit. Second, from all physical fields, which will appear in a future theory (according to present understanding), only the quantum component of gravitational field (graviton field) has a unique combination of zero rest mass and conformal non-invariance properties. Third, physically meaningful effects of quantum gravity can be

\footnotetext{
${ }^{1}$ Everywhere in this paper we discuss quantum states of gravitons and ghosts that are self-consistent with the evolution of macroscopic geometry of the Universe. In the mathematical formalism of the theory, the ghosts play a role of a second physical subsystem, the average contributions of which to the macroscopic Einstein equations appear on an equal basis with the average contribution of gravitons. At first glance, it may seem that the status of the ghosts as the second subsystem is in a contradiction with the well-known fact that the Faddeev-Popov ghosts are not physical particles. However the paradox, is in the fact that we have no contradiction with the standard concepts of quantum theory of gauge fields but rather full agreement with these. The Faddeev-Popov ghosts are indeed not physical particles in a quantum-field sense, that is they are not particles that are in the asymptotic states whose energy and momentum are connected by a definite relation. Such ghosts are nowhere to be found on the pages of our work. We discuss only virtual gravitons and virtual ghosts that exist in the area of interaction. As to virtual ghosts, they cannot be eliminated in principle due to lack of ghost-free gauges in quantum gravity. In the strict mathematical sense, the non-stationary Universe as a whole is a region of interaction, and, formally speaking, there are no real gravitons and ghosts in it. Approximate representations of real particles, of course, can be introduced for shortwave quantum modes. In our work, quantum states of shortwave ghosts are not introduced and consequently are not discussed. Furthermore, macroscopic quantum effects, which are discussed in our work, are formed by the most virtual modes of all virtual modes. These modes are selected by the equality $\lambda H=1$, where $\lambda$ is the wavelength, $H$ is the Hubble function. The same equality also characterizes the intensity of interaction of the virtual modes with the classical gravitational field, i.e. it reflects the essentially non-perturbative nature of the effects. An approximate transition to real, weakly interacting particles, situated on the mass shell is impossible for these modes, in principle (see also the footnote 2 on p. 4).
}

identified and quantified in one-loop approximation. Fourth, as was been shown by t'Hooft and Veltman [3], the oneloop quantum gravity with ghost sector and without fields of matter is finite. For the property of one-loop finiteness, proven in [3] on the graviton mass shell, we add the following key assertion. All one-loop calculations in quantum gravity must be done in such a way that the feature of one-loop finiteness (lack of divergences in terms of observables) must automatically be implemented not only on the graviton mass shell but also outside it.

Let us emphasize the following important fact. Because of conformal non-invariance and zero rest mass of gravitons, no conditions exist in the Universe to place gravitons on the mass shell precisely. Therefore, in the absence of one-loop finiteness, divergences arise in observables. To eliminate them, the Lagrangian of Einstein's theory must be modified, by amending the definition of gravitons. In other words, in the absence of oneloop finiteness, gravitons generate divergences, contrary to their own definition. Such a situation does not make any sense, so the one-loop finiteness off the mass shell is a prerequisite for internal consistency of the theory.

These four conditions provide for the reliability of theory predictions. Indeed, the existence of quantum component of the gravitational field leaves no doubt. Zero rest mass of this component means no threshold for quantum processes of graviton vacuum polarization and graviton creation by external or self-consistent macroscopic gravitational field. The combination of zero rest mass and conformal non-invariance of graviton field leads to the fact that these processes are occurring even in the isotropic Universe at any stage of its evolution, including the contemporary Universe. Vacuum polarization and particle creation belong to effects predicted by the theory already in one-loop approximation. In this approximation, calculations of quantum gravitational processes involving gravitons are not accompanied by the emergence of divergences. Thus, the one-loop finiteness of quantum gravity allows uniquely describe mathematically graviton contributions to the macroscopic observables. Other one-loop effects in the isotropic Universe are suppressed either because of conformal invariance of non-gravitational quantum fields, or (in the modern Universe) by non-zero rest mass particles, forming effective thresholds for quantum gravitational processes in the macroscopic self-consistent field.

Effects of vacuum polarization and particle creation in the sector of matter fields of $J=0,1 / 2,1$ spin were well studied in the 1970's by many authors (see [4] and references therein). The theory of classic gravitational waves in the isotropic Universe was formulated by Lifshitz in 1946 [5]. Grishchuk [6] considered a number of cosmological applications of this theory that are result 
of conformal non-invariance of gravitational waves. Isaacson $[7,8]$ has formulated the task of self-consistent description of gravitational waves and background geometry. The model of Universe consisting of short gravitational waves was described for the first time in $[9,10]$. The energy-momentum tensor of classic gravitational waves of super long wavelengths was constructed in [11, 12]. The canonic quantization of gravitational field was done in [13-15]. The local speed of creation of shortwave gravitons was calculated in [16]. In all papers listed above, the ghost sector of graviton theory was not taken into account. One-loop quantum gravity in the form of the theory of gravitons defined on the background spacetime was described by De Witt [17]. Calculating methods of this theory were discussed by Hawking [18].

The exact equations of self-consistent theory of gravitons in the Heisenberg representation with the ghost sector automatically providing a one-loop finiteness off the mass shell are obtained in our work [19]. In [19], it is shown that the Heisenberg representation of quantum gravity (as well as the Heisenberg representation of quantum Yang-Mills theory [20]) exists only in the Hamilton gauge. The ghost sector corresponding to this gauge represented by the complex scalar field with minimal coupling to gravity.

One-loop finiteness provides the simplicity and elegance of a mathematical theory that allows, in turn, discovering a number of new approximate and exact solutions of its equations. This paper is focused on three exact solutions corresponding to three different quantum states of graviton-ghost subsystem in the space of the non-stationary isotropic Universe with self-consistent geometry. The first of these solutions describes a coherent condensate of virtual gravitons and ghosts; the second solution describes a coherent condensate of instanton fluctuations. The third solution describes the self-polarized condensate in the De Sitter space. This solution allows interpretation in terms of virtual particles as well as in terms of instanton fluctuations.

The principal nature of macroscopic quantum gravity effects, the need for strict proof of their inevitability and reliability impose stringent requirements for constructing a mathematical algorithm of the theory. Sections 2 and 3 are devoted to the derivation of the equations of the theory with a discussion of all the mathematical details. In Section 2, we start with exact quantum theory of gravity, presented in terms of path integral of Faddeev-Popov [21] and De Witt [22,23]. Key ideas of this Section are the following. 1) The necessity to gauge the full metric (before its separation into the background and fluctuations) and the inevitability of appearance of a ghost sector in the exact path integral and operator Einstein's equations (Sections 2.1 and 2.2); 2) The principal necessity to use normal coordinates (exponential parameterization) in a mathematically rigorous procedure for the separation of classical and quantum variables is discussed in Sections 2.3 and 2.4 ; 3) The derivation of differential identities, providing the consistency of classical and quantum equations performed jointly in any order of the theory of perturbations is given Section 2.5. Rigorously derived equations of gauged one-loop quantum gravity are presented in Section 2.6.

The status of properties of ghost sector generated by gauge is crucial to properly assess the structure of the theory and its physical content. Let us immediately emphasize that the standard presentation on the ghost status in the theory of S-matrix can not be exported to the theory of gravitons in the macroscopic spacetime with selfconsistent geometry. Two internal mathematical properties of the quantum theory of gravity make such export fundamentally impossible. First, there are no gauges that completely eliminate the diffeomorphism group degeneracy in the theory of gravity. This means that among the objects of the quantum theory of fields inevitably arise ghosts interacting with macroscopic gravity. Secondly, gravitons and ghosts cannot be in principle situated precisely on the mass shell because of their conformal non-invariance and zero rest mass. This is because there are no asymptotic states, in which interaction of quantum fields with macroscopic gravity could be neglected. Restructuring of vacuum graviton and ghost modes with a wavelength of the order of the distance to the horizon of events takes place at all stages of cosmological evolution, including the contemporary Universe. Ghost trivial vacuum, understood as the quantum state with zero occupation numbers for all modes, simply is absent from physically realizable states. Therefore, direct participation of ghosts in the formation of macroscopic observables is inevitable ${ }^{2}$.

Section 3 is devoted to general discussion of equations of the theory of gravitons in the isotropic Universe. It focuses on three issues: 1) Canonical quantization of gravitons and ghosts (Sections 3.1 and 3.2); 2) Construction of the state vector of a general form as a product of normalized superpositions (Section 3.3); 3) The proof of the one-loop finiteness of macroscopic observables (Section 3.4). The main conclusion is that the quantum ghost fields are inevitable and unavoidable components of the quantum gravitational field. As noted above, one-loop finiteness is seen by us as a universal property of quantum gravity, which extends off the mass shell. The requirement of compensation of divergences in terms of macroscopic observables, resulting from oneloop finiteness, uniquely captures the dynamic properties of quantum ghost fields in the isotropic Universe. The existence of Quantum Gravity in the Heisenberg representation in the Hamilton gauge is a nontrivial property of the theory. Exactly this property automatically 
provides a one-loop finiteness of the theory off mass shell.

Sections 4.1 and 4.2 contain approximate solutions to obtain quantum ensembles of short and long gravitational waves. In Section 4.3 it is shown that approximate solutions obtained can be used to construct scenarios for the evolution of the early Universe. In one such scenario, the Universe is filled with ultra-relativistic gas of short-wave gravitons and with a condensate of super-long wavelengths, which is dominated by ghosts. The evolution of this Universe is oscillating in nature.

At the heart of cosmological applications of one-loop quantum gravity is the Bogolyubov-Born-Green-Kirckwood-Yvon (BBGKY) chain (or hierarchy) for the spectral function of gravitons, renormalized by ghosts. We present the first use of this technique in quantum gravity calculations. Each equation of the BBGKY chain connects the expressions for neighboring moments of the spectral function. In Section 5.1, the BBGKY chain is derived by identical mathematical procedures from graviton and ghost operator equations. Among these procedures is averaging of bilinear forms of field operators over the state vector of the general form, whose mathematical structure is given in Section 3.3. The need to work with state vectors of the general form is dictated by the instability of the trivial graviton-ghost vacuum (see [24], Section 3.6). Evaluation of mathematical correct-

${ }^{2}$ Once again, we emphasize that the equal participation of virtual gravitons and ghosts in the formation of macroscopic observables in the non-stationary Universe does not contradict the generally accepted concepts of the quantum theory of gauge fields. On the contrary it follows directly from the mathematical structure of this theory. In order to clear up this issue once and for all, recall some details of the theory of $S$-matrix. In constructing this theory, all space-time is divided into regions of asymptotic states and the region of effective interaction. Note that this decomposition is carried out by means of, generally speaking, an artificial procedure of turning on and off the interaction adiabatically. (For obvious reasons, the problem of self-consistent description of gravitons and ghosts in the non-stationary Universe with $\lambda H=1$ by means of an analogue of such procedure cannot be considered a priori.) Then, after splitting the space-time into two regions, it is assumed that the asymptotic states are ghost-free. In the most elegant way, this selection rule is implemented in the BRST formalism, which shows that the BRST invariant states turn out to be gauge-invariant automatically. The virtual ghosts, however, remain in the area of interaction, and this points to the fact that virtual gravitons and ghosts are parts of the Feynman diagrams on an equal footing. In the self-consistent theory of gravitons in the non-stationary Universe, virtual ghosts of equal weight as the gravitons, appear at the same place where they appear in the theory of $S$-matrix, i.e. at the same place as they were introduced by Feynman, i.e. in the region of interaction. Of course, the fact that in the real non-stationary Universe, both the observer and virtual particles with $\lambda H=1$ are in the area of interaction, is highly nontrivial. It is quite possible that this property of the real world is manifested in the effect of dark energy. An active and irremovable participation of virtual ghosts in the formation of macroscopic properties of the real Universe poses the question of their physical nature. Today, we can only say with certainty that the mathematical inevitability of ghosts provides the one-loop finiteness off the mass shell, i.e. the mathematical consistency of one-loop quantum gravity without fields of matter. Some hypothetical ideas about the nature of the ghosts are briefly discussed in the final Section 8 . ness of procedures for BBGKY structure is entirely a question of the existence of moments of the spectral function as mathematical objects. A positive answer to this question is guaranteed by one-loop finiteness (Section 3.4). The set of moments of the spectral function contains information on the dynamics of operators as well as on the properties of the quantum state over which the averaging is done. The set of solutions of BBGKY chain contains all possible self-consistent solutions of operator equation, averaged over all possible quantum ensembles.

A nontrivial fact is that in the one-loop quantum gravity $B B G K Y$ chain can formally be introduced at an axiomatic level. Theory of gravitons provided by BBGKY chain, conceptually and mathematically corresponds to the axiomatic quantum field theory in the Wightman formulation (see Chapter 8 in the monograph [25]). Here, as in Wightman, the full information on the quantum field is contained in an infinite sequence of averaged correlation functions. Definitions of these functions clearly relate to the symmetry properties of manifold on one this field is defined. Once the BBGKY chain is set up, the existence of finite solutions for the observables is provided by inherent mathematical properties of equations of the chain. This means that the phenomenology of BBGKY chain is more general than field operators, state vectors and graviton-ghost compensation of divergences that were used in its derivation.

Exact solutions of the equations, consisting of BBGKY chain and macroscopic Einstein's equations are obtained in Sections 5.2 and 5.3. Two solutions given in 5.2, describe heterogeneous graviton-ghost condensates, consisting of three subsystems. Two of these are condensates of spatially homogeneous modes with the equations of state $p=-\varepsilon / 3$ and $p=\varepsilon$. The third subsystem is a condensate of quasi-resonant modes with a constant conformal wavelength corresponding to the variable physical wavelength of the order of the distance to the horizon of events. The equations of state of condensates of quasiresonant modes differ from $p \sim-\varepsilon / 3$ by logarithmic terms, through which the first solution is $p \gtrsim-\varepsilon / 3$, while the second is $p \lesssim-\varepsilon / 3$. Furthermore, the solutions differ by the sign of the energy density of condensates of spatially homogenous modes. The third solution describes a homogeneous condensate of quasi-resonant modes with a constant physical wavelength. The equation of state of this condensate is $p=-\varepsilon$ and its selfconsistent geometry is the De Sitter space. The three solutions are interpreted as three different phase states of graviton-ghost system. The problem of quantum-gravity phase transitions is discussed in Section 5.4.

Solutions obtained in Section 5 in terms of moments of the spectral function, are reproduced in Sections 6 and 7 at the level of dynamics of operators and state vectors. A 
microscopic theory provides details to clarify the structure of graviton-ghost condensates and clearly demonstrates the effects of quantum interference of coherent states. In Section 6.1, it is shown that the condensate of quasi-resonant modes with the equation of state

$p \gtrsim-\varepsilon / 3$ consists of virtual gravitons and ghosts. In Section 6.2 a similar interpretation is proposed for the condensate in the De Sitter space, but it became necessary to extend the mathematical definition of the moments of the spectral function.

New properties of the theory, whose existence was not anticipated in advance, are studied in Section 7. In Section 7.1 we find that the self-consistent theory of gravitons and ghosts is invariant with respect to the Wick turn. In this section, we also construct the formalism of quantum theory in the imaginary time and discuss the physical interpretation of this theory. The subjects of the study are correlated fluctuations arising in the process of tunnelling between degenerate states of graviton-ghost systems, divided by classically impenetrable barriers. The level of these fluctuations is evaluated by instanton solutions (as in Quantum Chromodynamics). In Section 7.2, it is shown that the condensate of quasi-resonant modes with the equation of state $p \lesssim-\varepsilon / 3$ is of purely instanton nature. In Section 7.3, the instanton condensate theory is formulated for the De Sitter space.

Potential use of the results obtained to construct scenarios of cosmological evolution was briefly discussed in Sections 4-7 to obtain approximate and exact solutions. Future issues of the theory of the theory are briefly discussed in the Conclusion (Section 8).

A system of units is used, in which the speed of light is $c=1$, Planck constant is $\hbar=197.327 \mathrm{MeV} \cdot \mathrm{fm}$; Einstein's gravity constant is $\varkappa \equiv 8 \pi G=8 \pi \cdot 1.324 \times 10^{-42} \mathrm{MeV}^{-1} \cdot \mathrm{fm}$.

\section{Basic Equations}

According to De Witt [17], one of formulations of oneloop quantum gravity (with no fields of matter) is reduced to the zero rest mass quantum field theory with spin $J=2$, defined for the background spacetime with classic metric. The graviton dynamics is defined by the interaction between quantum field and classic gravity, and the background space geometry, in turn, is formed by the energy-momentum tensor (EMT) of gravitons.

In the current Section we describe how to get the selfconsistent system of equations, consisting of quantum operator equations for gravitons and ghosts and classic $C$-number Einstein equations for macroscopic metrics with averaged EMT of gravitons and ghosts on the right hand side. The theory is formulated without any constrains on the graviton wavelength that allows the use of the theory for the description of quantum gravity effects at the long wavelength region of the specter. The equ- ations of the theory (except the gauge condition) are represented in $4 \mathrm{D}$ form which is general covariant with respect to the transformation of the macroscopic metric.

The mathematically consistent system of 4D quantum and classic equations with no restrictions with respect to graviton wavelengths is obtained by a regular method for the first time. The case of a gauged path integral with ghost sector is seen as a source object of the theory. Important elements of the method are exponential parameterization of the operator of the density of the contravariant metric; factorization of path integral measure; consequent integration over quantum and classic components of the gravitational field. Mutual compliance of quantum and classic equations, expressed in terms of fulfilling of the conservation of averaged EMT at the operator equations of motion is provided by the virtue of the theory construction method.

\subsection{Path Integral and Faddeev-Popov Ghosts}

Formally, the exact scheme of quantum gravity is based on the amplitude of transition, represented by path integral $[21,22]$ :

$$
\begin{aligned}
\langle\text { out }| \text { in }\rangle= & \int \exp \left(\frac{i}{\hbar} \int\left(L_{\text {grav }}+L_{\Lambda}\right) \mathrm{d}^{4} x\right)\left(\operatorname{det} \hat{M}_{k}^{i}\right) \\
& \times \prod_{x}\left(\prod_{i} \delta\left(\hat{A}_{k} \sqrt{-\hat{g}} \hat{g}^{i k}-B^{i}\right)\right) \mathrm{d} \hat{\mu} \\
= & \int \exp \left(\frac{i}{\hbar} \int\left(L_{\text {grav }}+L_{\Lambda}+L_{\text {ghost }}\right) \mathrm{d}^{4} x\right) \\
& \times \prod_{x}\left(\prod_{i} \delta\left(\hat{A}_{k} \sqrt{-\hat{g}} \hat{g}^{i k}-B^{i}\right)\right) \mathrm{d} \hat{\mu} \mathrm{d} \mu_{\theta},
\end{aligned}
$$

where

$$
L_{\text {grav }}+L_{\Lambda}=-\frac{1}{2 \varkappa} \sqrt{-\hat{g}} \hat{g}^{i k} \hat{R}_{i k}-\sqrt{-\hat{g}} \Lambda
$$

is the density of gravitational Lagrangian, with cosmological constant included; $L_{\text {ghost }}$ is the density of ghost Lagrangian, explicit form of which is defined by localization of $\operatorname{det} \hat{M}_{k}^{i} ; \hat{A}_{k}$ is gauge operator, $B^{i}(x)$ is the given field; $\hat{M}_{k}^{i}$ is an operator of equation for infinitesimal parameters of transformations for the residual degeneracy $\eta^{i}=\delta x^{i}$;

$$
\mathrm{d} \hat{\mu}=\prod_{x}\left\{(-\hat{g})^{5 / 2} \prod_{i \leq k} \mathrm{~d} \hat{g}^{i k}\right\}
$$

is the gauge invariant measure of path integration over gravitational variables; $d \mu_{\theta}$ is the measure of integration over ghost variables. Operator $\hat{M}_{k}^{i}$ is of standard definition:

$$
\hat{M}_{k}^{i} \eta^{k} \equiv \hat{A}_{k}\left(\delta \sqrt{-\hat{g}} \hat{g}^{i k}\right)=0,
$$

where 


$$
\begin{aligned}
\delta \sqrt{-\hat{g}} \hat{g}^{i k} & =-\partial_{l}\left(\sqrt{-\hat{g}} \hat{g}^{i k} \eta^{l}\right) \\
& +\sqrt{-\hat{g}} \hat{g}^{i l} \partial_{l} \eta^{k}+\sqrt{-\hat{g}} \hat{g}^{k l} \partial_{l} \eta^{i}
\end{aligned}
$$

is variation of metrics under the action of infinitesimal transformations of the group of diffeomorphisms. According to (1), the allowed gauges are constrained by the condition of existence of the inverse operator

$$
\left(\hat{M}_{k}^{i}\right)^{-1} \text {. }
$$

The Equation (1) explicitly manifests the fact that the source path integral is defined as a mathematical object only after the gauge has been imposed. In the theory of gravity, there are no gauges completely eliminating the degeneracy with respect to the transformations (4). Therefore, the sector of nontrivial ghost fields, interacting with gravity, is necessarily present in the path integral. This aspect of the quantum gravity is important for understanding of its mathematical structure, which is fixed before any approximations are introduced. By that reason, in this Section we discuss the equations of the theory, by explicitly defining the concrete gauge.

The mathematical procedure of transition from path integral (1) to the equations of Quantum Gravity in the Heisenberg representation (with the canonical quantization of gravitons and ghosts) is described by us in detail [19]. The first step of this procedure is to represent the integral (1) as a path integral over the canonical variables. Such an integral was proposed by Faddeev [26] on the basis of the general theory of Hamilton systems with explicitly unsolvable constraints [27]. The second step is to introduce the normal coordinates of the gravitational field using the exponential parameterization of metric. The Hamilton gauge of the normal coordinates specifies the Faddeev path integral in such a way that the ghost sector (corresponding to it) allows to introduce canonical variables of ghost fields and to represent the ghost Lagrangian in the Hamilton form. In the third step of this procedure, the transition from the gauged path integral to the canonical Hamilton formalism in the Heisenberg representation is made (using the standard definition of the operator of evolution). The results of the [19] are rigorous basis of the simplified procedure for obtaining gauged equations of quantum gravity with ghosts, which is described below.

Hamilton gauge is that of synchronous type:

$$
\sqrt{-\hat{g}} \hat{g}^{00}=\sqrt{\bar{\gamma}}, \quad \sqrt{-\hat{g}} \hat{g}^{0 \alpha}=0
$$

For that gauge

$$
\hat{A}_{k}=(1,0,0,0), \quad B^{i}=(\sqrt{\bar{\gamma}}, 0,0,0),
$$

where $\bar{\gamma}=\bar{\gamma}(\boldsymbol{x})$ is the metric determinant of the basic 3D space of constant curvature (for the plane cosmological model $\bar{\gamma}=1$ ).
The construction of the ghost sector, i.e. finding of the Lagrangian density $L_{\text {ghost }}$, is reduced to two operations. First, det $\hat{M}_{k}^{i}$ is represented in the form, factorized over independent degrees of freedom for ghosts, and then the localization of the obtained expression is conducted. Substitution of (6) and (4) to (3) gives the following system of equations

$$
\begin{aligned}
& -\partial_{\alpha} \sqrt{\bar{\gamma}} \eta^{\alpha}+\sqrt{\bar{\gamma}} \frac{\partial \eta^{0}}{\partial t}=0, \\
& \sqrt{-\hat{g}} \hat{g}^{\alpha \beta} \partial_{\beta} \eta^{0}+\frac{\partial \sqrt{\bar{\gamma}} \eta^{\alpha}}{\partial t}=0 .
\end{aligned}
$$

According to (7), with respect to variables $\eta^{0}, \sqrt{\bar{\gamma}} \eta^{\alpha}$ the operator-matrix $\hat{M}_{k}^{i}$ reads

$$
\hat{M}_{k}^{i}=\left(\begin{array}{cc}
\sqrt{\bar{\gamma}} \frac{\partial}{\partial t} & -\partial_{\alpha} \\
\sqrt{-\hat{g}} \hat{g}^{\alpha \beta} \partial_{\beta} & \delta_{\beta}^{\alpha} \frac{\partial}{\partial t}
\end{array}\right)
$$

(Note matrix-operator is obtained in the form (8) without the substitution of transformation parameters if Leutwiller measure $\mathrm{d} \hat{\mu}_{L}=\hat{g} \hat{g}^{00} \mathrm{~d} \hat{\mu}$ is used. The measure discussion see, e.g. [28].) Functional determinant of matrixoperator det $\hat{M}_{k}^{i}$ is represented in the form of the determinant of matrix $\hat{M}_{k}^{i}$, every element of which is a functional determinant of differential operator. As it is follows from (8),

$$
\operatorname{det} \hat{M}_{k}^{i}=\left(\operatorname{det} \partial_{i} \sqrt{\hat{g}} \hat{g}^{i k} \partial_{k}\right) \times\left(\operatorname{det} \frac{\partial}{\partial t}\right) \times\left(\operatorname{det} \frac{\partial}{\partial t}\right) \text {. }
$$

One can see that the first multiplier in (9) is 4-invariant determinant of the operator of the zero rest mass Klein-Gordon-Fock equation, and two other multipliers do not depend on gravitational variables.

Localization of determinant (9) by representing it in a form of path integral over the ghost fields is a trivial operation. As it follows from (9), the class of synchronous gauges contains three dynamically independent ghost fields $\theta, \varphi, \chi$, two of each $\varphi, \chi$ do not interact with gravity. For the obvious reason, the trivial ghosts $\varphi, \chi$ are excluded from the theory. The Lagrangian density of nontrivial ghosts coincides exactly with Lagrangian density of complex Klein-Gordon-Fock fields (taking into account the Grassman character of fields $\bar{\theta}, \theta)$ :

$$
L_{\text {ghost }}=-\frac{1}{4 \varkappa} \sqrt{-\hat{g}} \hat{g}^{i k} \partial_{i} \bar{\theta} \cdot \partial_{k} \theta
$$

The normalization multiplier $-1 / 4 \varkappa$ in (10) is chosen for the convenience. The integral measure over ghost fields has a simple form:

$$
\mathrm{d} \mu_{\theta}=\prod_{x} \mathrm{~d} \bar{\theta} \mathrm{d} \theta
$$


The calculations above comply with both general requirements to the construction of ghost sector. First, path integration should be carried out only over the dynamically independent ghost fields. Second, in the ghost sector, it is necessary to extract and then to take into account only the nontrivial ghost fields, i.e. those interacting with gravity.

\subsection{Einstein Operator Equations}

Let us take into account the fact that the calculation of gauged path integral should be mathematically equivalent to the solution of dynamical operator equations in the Heisenberg representation. It is also clear that operator equations of quantum theory should have a definite relationship with Einstein equations. In the classic theory, it is possible to use any form of representation of Einstein equations, e.g.

$$
\begin{aligned}
& (-\hat{g})^{n}\left(\hat{g}^{i l} \hat{g}^{k m} \hat{R}_{l m}-\frac{1}{2} \hat{g}^{i k} \hat{g}^{l m} \hat{R}_{l m}-\hat{g}^{i k} \varkappa \Lambda\right)=0,(a) \\
& (-\hat{g})^{n}\left(\hat{g}^{k m} \hat{R}_{i m}-\frac{1}{2} \delta_{i}^{k} \hat{g}^{l m} \hat{R}_{l m}-\delta_{i}^{k} \varkappa \Lambda\right)=0, \quad(b) \\
& (-\hat{g})^{n}\left(\hat{R}_{i k}-\frac{1}{2} \hat{g}_{i k} \hat{g}^{l m} \hat{R}_{l m}-\hat{g}^{i k} \varkappa \Lambda\right)=0, \quad(c)
\end{aligned}
$$

where, for example, $n=0,1 / 2,1$. Transition from one to another is reduced to the multiplication by metric tensor and its determinant, which are trivial operations in case when the metric is a $C$-number function. If the metric is an operator, then the analogous operations will, at least, change renormalization procedures of quantum non-polynomial theory. Thus, the question about the form of notation for Einstein's operator equations has first-hand relation to the calculation procedure. Now we show that in the quantum theory one should use operator equations (11b) with $n=1 / 2$, supplemented by the energy-momentum pseudo-tensor of ghosts.

In the path integral formalism, the renormalization procedures are defined by the dependence of Lagrangian of interactions and the measure of integration of the field operator in terms of which the polynomial expansion of non-polynomial theory is defined [29]. The introduction of such an operator, i.e. the parameterization of the metric, is, generally speaking, not simple. Nevertheless, it is possible to find a special parameterization for which the algorithms of renormalization procedures are defined only by Lagrangian of interactions. Obviously, in such a parameterization the measure of integration should be trivial. It reads:

$$
\mathrm{d} \hat{\mu}=\prod_{x} \prod_{i \leq k} \mathrm{~d} \hat{\Psi}_{i}^{k},
$$

where $\hat{\Psi}_{i}^{k}$ is a dynamic variable. The metric is expressed via this variable. It is shown in [29] that the trivialization of measure (12) takes place for the exponential parameterization that reads

$$
\begin{aligned}
\sqrt{-\hat{g}} \hat{g}^{i k} & =\sqrt{-\bar{g}} \bar{g}^{i l}(\exp \hat{\Psi})_{l}^{k} \\
& =\sqrt{-\bar{g}} \bar{g}^{i l}\left(\delta_{l}^{k}+\hat{\Psi}_{l}^{k}+\frac{1}{2} \hat{\Psi}_{l}^{m} \hat{\Psi}_{m}^{k}+\cdots\right),
\end{aligned}
$$

where $\bar{g}^{i k}$ is the defined metric of an auxiliary basic space. In that class of our interest, the metric is defined by the interval

$$
d \bar{s}^{2}=d t^{2}-\bar{\gamma}_{\alpha \beta} d x^{\alpha} d x^{\beta}
$$

where $\bar{\gamma}_{\alpha \beta}$ is the metric of 3D space with a constant curvature. (For the flat Universe $\bar{\gamma}_{\alpha \beta}$ is the Euclid metric.)

The exponential parameterization is singled out among all other parameterizations by the property that $\hat{\Psi}_{i}^{k}$ are the normal coordinates of gravitational fields [30]. In that respect, the gauge conditions (5) are identical to $\hat{\Psi}_{0}^{i}=0$. The fact that the "gauged" coordinates are the normal coordinates, leads to a simple and elegant ghost sector (10). The status of $\hat{\Psi}_{i}^{k}$, as normal coordinates, is of principal value for the mathematical correctness while separating the classic and quantum variables (see Section 2.4). Besides, in the framework of perturbation theory the normal coordinates allow to organize a calculation procedure, which is based on a simple classification of nonlinearity of quantum gravity field. It is important that this procedure is mathematically non-contradictive at every order of perturbation theory over amplitude of quantum fields (see Section 2.5 and 2.6).

Operator Einstein equations that are mathematically equivalent to the path integral of a trivial measure are derived by the variation of gauged action by variables $\hat{\Psi}_{i}^{k}$. The principal point is that the gauged action necessarily includes the ghost sector because there are no gauges that are able to completely eliminate the degeneracy. According to (10), in the Hamilton gauge we get

$S$

$$
=-\int \mathrm{d}^{4} x\left\{\frac{1}{2 \varkappa} \sqrt{-\hat{g}} \hat{g}^{i k}\left(\hat{R}_{i k}+\frac{1}{2} \partial_{i} \bar{\theta} \cdot \partial_{k} \theta\right)+\sqrt{-\hat{g}} \Lambda\right\} .
$$

In accordance with definition (13), the variation is done by the rule

$$
\delta \sqrt{-\hat{g}} \hat{g}^{i k}=\sqrt{-\hat{g}} \hat{g}^{i l} \delta \hat{\Psi}_{l}^{k} .
$$

Thus, from (14) it follows

$$
\begin{aligned}
\hat{\mathcal{G}}_{i}^{k} \equiv & \sqrt{-\hat{g}} \hat{g}^{k l} \hat{R}_{i l}+\varkappa \sqrt{-\hat{g}} \delta_{i}^{k} \Lambda \\
& -\varkappa\left(\sqrt{-\hat{g}} \hat{g}^{k l} \hat{T}_{i l}^{\text {(ghost })}-\frac{1}{2} \delta_{i}^{k} \sqrt{-\hat{g}} \hat{g}^{m l} \hat{T}_{m l}^{(\text {ghost })}\right)=0 .
\end{aligned}
$$

After subtraction of semi-contraction from (15) we obtain a mathematically equivalent equation 


$$
\begin{aligned}
\hat{\mathcal{E}}_{i}^{k}= & \hat{\mathcal{G}}_{i}^{k}-\frac{1}{2} \delta_{i}^{k} \hat{\mathcal{G}}_{l}^{l} \\
\equiv & \sqrt{-\hat{g}} \hat{g}^{k l} \hat{R}_{i l}-\frac{1}{2} \delta_{i}^{k} \sqrt{-\hat{g}} \hat{g}^{m l} \hat{R}_{m l} \\
& -\varkappa\left(\sqrt{-\hat{g}} \hat{g}^{k l} \hat{T}_{i l}^{(\text {ghost })}+\sqrt{-\hat{g}} \delta_{i}^{k} \Lambda\right)=0 .
\end{aligned}
$$

In (15), (16) there is an object

$$
\begin{aligned}
& \hat{T}_{i k}^{(\text {ghost })} \\
& =-\frac{1}{4 \varkappa}\left(\partial_{i} \bar{\theta} \cdot \partial_{k} \theta+\partial_{k} \bar{\theta} \cdot \partial_{i} \theta-\hat{g}_{i k} \hat{g}^{l m} \partial_{l} \bar{\theta} \cdot \partial_{m} \theta\right),
\end{aligned}
$$

which has the status of the energy-momentum pseudotensor of ghosts.

In accordance with the general properties of Einstein's theory, six spatial components of Equations (15) are considered as quantum equations of motion:

$$
\begin{aligned}
& \sqrt{-\hat{g}} \hat{g}^{\beta l} \hat{R}_{\alpha l}+\varkappa \sqrt{-\hat{g}} \delta_{\alpha}^{\beta} \Lambda \\
& =\varkappa\left(\sqrt{-\hat{g}} \hat{g}^{\beta l} \hat{T}_{\alpha l}^{(\text {ghost })}-\frac{1}{2} \delta_{\alpha}^{\beta} \sqrt{-\hat{g}} \hat{g}^{m l} \hat{T}_{m l}^{(\text {ghost })}\right) .
\end{aligned}
$$

(Everywhere in this work the Greek metric indexes stand for $\alpha, \beta=1,2,3$.) In the classic theory, equations of constraints $\hat{\mathcal{E}}_{0}^{0}=0$ and $\hat{\mathcal{E}}_{0}^{\alpha}=0$ are the first integrals of equations of motion (18). Therefore, in the quantum theory formulated in the Heisenberg representation four primary constraints from (16), have the status of the initial conditions for the Heisenberg state vector. They read:

$$
\begin{aligned}
& \left\{\sqrt{-\hat{g}} \hat{g}^{0 l} \hat{R}_{0 l}-\frac{1}{2} \sqrt{-\hat{g}} \hat{g}^{m l} \hat{R}_{m l}\right. \\
& \left.-\varkappa\left(\sqrt{-\hat{g}} \hat{g}^{0 l} \hat{T}_{0 l}^{(\text {ghost })}+\sqrt{-\hat{g}} \Lambda\right)\right\}|\Psi\rangle=0, \\
& \left\{\sqrt{-\hat{g}} \hat{g}^{\alpha l} \hat{R}_{0 l}-\varkappa \sqrt{-\hat{g}} \hat{g}^{\alpha l} \hat{T}_{0 l}^{(\text {ghost })}\right\}|\Psi\rangle=0 .
\end{aligned}
$$

If conditions (19) are valid from the start, then the internal properties of the theory must provide their validity at any subsequent moment of time. Four secondary relations, defined by the gauge non containing the higher order derivatives, also have the same status:

$$
\left\{\hat{A}_{k}\left(\sqrt{-\hat{g}} \hat{g}^{i k}\right)-B^{i}\right\}|\Psi\rangle=0 \text {. }
$$

The system of equations of quantum gravity is closed by the ghosts' equations of motion, obtained by the variation of action (14) over ghost variables:

$$
\begin{aligned}
& \partial_{i} \sqrt{-\hat{g}} \hat{g}^{i k} \partial_{k} \theta=0, \\
& \partial_{i} \sqrt{-\hat{g}} \hat{g}^{i k} \partial_{k} \bar{\theta}=0 .
\end{aligned}
$$

Ghost fields $\bar{\theta}$ and $\theta$ are not defined by Grassman scalars, therefore $T_{i k}^{\text {(ghost) }}$ is not a tensor. Nevertheless, all mathematical properties of Equation (21) and ex- pressions (17) coincide with the respected properties of equations and EMT of complex scalar fields. This fact is of great importance when concrete calculations are done (see Section 3).

\subsection{Factorization of the Path Integral}

Transition from the formally exact scheme (18)-(21)) to the semi-quantum theory of gravity can be done after some additional hypotheses are included in the theory. The physical content of these hypotheses consists of the assertion of existence of classical spacetime with metric $g_{i k}$, connectivity $\Gamma_{k l}^{i}$ and curvature $R_{i k}$. The first hypothesis is formulated at the level of operators. Assume that operator of metric $\hat{g}^{i k}$ is a functional of $C$-number function $g^{i k}$ and the quantum operator $\hat{\psi}_{i}^{k}$. The second hypothesis is related to the state vector. Each state vector that is involved in the scalar product $\langle$ out $|$ in $\rangle$, is represented in a factorized form $|\Psi\rangle=|\Phi\rangle|\psi\rangle$, where $|\Psi\rangle$ are the vectors of quantum states of gravitons; $|\Phi\rangle$ are the vectors of quasi-classic states of macroscopic metric. In the framework of these hypotheses the transitional amplitude is reduced to the product of amplitudes:

$$
\langle\text { out }| \text { in }\rangle=\left\langle\Phi_{\text {out }} \mid \Phi_{\text {in }}\right\rangle\left\langle\psi_{\text {out }} \mid \psi_{\text {in }}\right\rangle .
$$

Thus, the physical assumption about existence of classic spacetime formally (mathematically) means that the path integral must be calculated first by exact integration over quantum variables, and then by approximate integration over the classic metric.

Mathematical definition of classic and quantum variables with subsequent integrations are possible only after the trivialization and factorization of integral measure are done. As already noted, trivial measure (12) takes place in exponential parameterization (13). The existence of $\mid$ in $\rangle=|\Psi\rangle$ vector allows the introduction of classic $C$ number variables as follows

$$
\Phi_{i}^{k}=\left\langle\Psi\left|\hat{\Psi}_{i}^{k}\right| \Psi\right\rangle, \quad \sqrt{-g} g^{i k}=\sqrt{-\bar{g}} \bar{g}^{i l}(\exp \Phi)_{l}^{k} .
$$

Quantum graviton operators are defined as the difference $\hat{\psi}_{i}^{k}=\hat{\Psi}_{i}^{k}-\Phi_{i}^{k}$. Factorized amplitude (22) is calculated via the factorized measure

$$
\begin{aligned}
& d \hat{\mu}=d \mu_{g} \times d \mu_{\psi}, \\
& d \mu_{g}=\prod_{x}\left\{(-g)^{5 / 2} \prod_{i \leq k} d g^{i k}\right\}, \quad d \mu_{\psi}=\prod_{x} \prod_{i \leq k} d \hat{\psi}_{i}^{k} .
\end{aligned}
$$

Factorization of the measure allows the subsequent integration, first by $d \mu_{\psi}, d \mu_{\theta}$, then by approximate integration over $d \mu_{g}$. In the operator formalism, such consecutive integrations correspond to the solution of self-consistent system of classic and quantum equations. Classical equations are obtained by averaging of operator Equation (16). They read: 


$$
\left\langle\Psi\left|\hat{\mathcal{E}}_{i}^{k}\right| \Psi\right\rangle=0 .
$$

Subtraction of (24) from (16) gives the quantum dynamic equations

$$
\hat{\mathcal{E}}_{i}^{k}-\left\langle\Psi\left|\hat{\mathcal{E}}_{i}^{k}\right| \Psi\right\rangle=0 .
$$

Synchronous gauge (20) is converted to the gauge of classical metric and to conditions imposed on the state vector:

$$
\sqrt{-g} g^{00}=\sqrt{\bar{\gamma}}, \sqrt{-g} g^{0 \alpha}=0, \hat{\psi}_{0}^{i}|\Psi\rangle=0 .
$$

Quantum Equation (21) of ghosts' dynamics are added to Equations (24)-(26).

Theory of gravitons in the macroscopic spacetime with self-consistent geometry is without doubt an approximate theory. Formally, the approximation is in the fact that the single mathematical object $\sqrt{-\hat{g}} \hat{g}^{i k}$ is replaced by two objects - classical metric and quantum field, having essentially different physical interpretations. That "coercion" of the theory can lead to a controversy, i.e. to the system of equations having no solutions, if an inaccurate mathematics of the adopted hypotheses is used. The scheme described above does not have such a controversy. The most important element of the scheme is the exponential parameterization (13), which separates the classical and quantum variables, as can be seen from (23). After the background and quantum fluctuations are introduced, this parameterization looks as follows:

$$
\begin{aligned}
\sqrt{-\hat{g}} \hat{g}^{i k} & =\sqrt{-\bar{g}} \bar{g}^{i l}(\exp (\Phi+\hat{\psi}))_{l}^{k} \\
& =\sqrt{-g} g^{i l}(\exp \hat{\psi})_{l}^{k},
\end{aligned}
$$

Note that the auxiliary basic space vanishes from the theory, and instead the macroscopic (physical) spacetime with self-consistent geometry takes its place.

If the geometry of macroscopic spacetime satisfies symmetry constrains, the factorization of the measure (23) becomes not a formal procedure but strictly mathematical in its nature. These restrictions must ensure the existence of an algorithm solving the equations of constraints in the framework of the perturbation theory (over the amplitude of quantum fields). The theory of gravity is non-polynomial, so after the separation of single field into classical and quantum components, the use of the perturbation theory in the quantum sector becomes unavoidable. The classical sector remains non-perturbative. In the general case, when quantum field is defined in an arbitrary Riemann space, the equations of constraints is not explicitly solvable. The problem can be solved in the framework of perturbation theory if background $g_{i k}$ and the free (linear) tensor field $\hat{\psi}_{i}^{k}$ belong to different irreducible representations of the symmetry group of the background spacetime. In that case at the level of linear field we obtain (23), because the full measure is represented as a product of measure of integration over independent irreducible representations. At the next order, factorization is done over coordinates, because the classical background and the induced quantum fluctuations have essentially different spacetime dynamics. Note, to factorize the measure by symmetry criterion we do not need to go to the short-wave approximation.

Background metric of isotropic cosmological models and classical spherically symmetric non-stationary gravitational field meet the constrains described above. These two cases are covering all important applications of semi-quantum theory of gravity which are quantum effects of vacuum polarization and creation of gravitons in the non-stationary Universe and in the neighborhood of black holes.

\subsection{Variational Principle for Classic and Quantum Equations}

Geometrical variables can be identically transformed to the form of functionals of classical and quantum variables. At the first step of transformation there is no need to fix the parameterization. Let us introduce the notations:

$$
\begin{aligned}
& \sqrt{-\hat{g}} \hat{g}^{i k}=\sqrt{-g} \hat{X}^{i k}, \frac{1}{\sqrt{-\hat{g}}} \hat{g}_{i k}=\frac{1}{\sqrt{-g}} \hat{Y}_{i k}, \\
& \hat{Y}_{i l} \hat{X}^{l k}=\delta_{i}^{k} .
\end{aligned}
$$

According to (27), formalism of the theory allows definition of quantum field $\hat{\psi}_{i}^{k}$ as symmetric tensor in physical space, $g_{k l} \hat{\psi}_{k}^{l}=\hat{\psi}_{i k}=\hat{\psi}_{k i}$. Objects, introduced in (28), have the same status. With any parameterization the following relationships take place:

$$
\lim _{\hat{\psi}_{l}^{m} \rightarrow 0} \hat{X}^{i k}=g^{i k}, \lim _{\hat{\psi}_{l}^{m} \rightarrow 0} \hat{Y}_{i k}=g_{i k} .
$$

We should also remember that the mixed components of tensors $\hat{X}_{i}^{k}, \hat{Y}_{i}^{k}$ do not contain the background metric as functional parameters. For any parameterization, these tensors are only functionals of quantum fields $\hat{\psi}_{i}^{k}$ which are also defined in mixed indexes. For the exponential parameterization:

$$
\begin{aligned}
& \hat{X}_{i}^{k}=\delta_{i}^{k}+\hat{\psi}_{i}^{k}+\frac{1}{2} \hat{\psi}_{i}^{l} \hat{\psi}_{l}^{k}+\cdots, \\
& \hat{Y}_{i}^{k}=\delta_{i}^{k}-\hat{\psi}_{i}^{k}+\frac{1}{2} \hat{\psi}_{i}^{l} \hat{\psi}_{l}^{k}+\cdots, \\
& \hat{g}=g \cdot \hat{d}=g \mathrm{e}^{\hat{\psi}},
\end{aligned}
$$

where $d=\operatorname{det}\left\|\hat{X}_{k}^{i}\right\|$. One can seen from (29), that the determinant of the full metric contains only the trace of the quantum field.

Regardless of parameterization, the connectivity and curvature of the macroscopic space $\Gamma_{i k}^{l}, R_{k l m}^{i}$ are extracted from full connectivity and curvature as additive 
terms:

$$
\hat{\Gamma}_{i k}^{l}=\Gamma_{i k}^{l}+\hat{\mathcal{T}}_{i k}^{l}, \quad \hat{R}_{k l m}^{i}=R_{k l m}^{i}+\hat{\mathcal{R}}_{k l m}^{i} .
$$

Quantum contribution to the curvature tensor,

$$
\hat{\mathcal{R}}_{k l m}^{i}=\hat{\mathcal{T}}_{k m ; l}^{i}-\hat{\mathcal{T}}_{k l ; m}^{i}+\hat{\mathcal{T}}_{n l}^{i} \hat{\mathcal{T}}_{k m}^{n}-\hat{\mathcal{T}}_{n m}^{i} \hat{\mathcal{T}}_{k l}^{n},
$$

is expressed via the quantum contribution to the full connectivity:

$$
\begin{aligned}
\hat{\mathcal{T}}_{i k}^{l}= & \frac{1}{2}\left(-\hat{Y}_{i m} \hat{X}_{; k}^{m l}-\hat{Y}_{k m} \hat{X}_{; i}^{m l}+\hat{Y}_{i j} \hat{Y}_{k n} \hat{X}^{m l} \hat{X}_{; m}^{j n}\right) \\
& +\frac{1}{4} Y_{j n}\left(\delta_{i}^{l} X_{; k}^{j n}+\delta_{k}^{l} X_{; i}^{j n}-Y_{i k} X^{m l} X_{; m}^{j n}\right) .
\end{aligned}
$$

The density of Ricci tensor in mixed indexes reads

$$
\begin{aligned}
& \sqrt{-\hat{g}} \hat{g}^{k l} \hat{R}_{i l} \\
& =\sqrt{-g}\left\{\hat{X}^{k l} R_{i l}\right. \\
& +\frac{1}{2}\left[\hat{Y}_{i n}\left(\hat{X}^{m l} \hat{X}_{; m}^{n k}-\hat{X}^{m k} \hat{X}_{; m}^{n l}\right)-\hat{X}_{; i}^{l k}-\frac{1}{2} \delta_{i}^{k} \hat{Y}_{n j} \hat{X}_{; m}^{m l} \hat{X}_{; j}^{j n}\right]_{; l} \\
& \left.-\frac{1}{4}\left(\hat{Y}_{j n} \hat{Y}_{s m}-\frac{1}{2} \hat{Y}_{j m} \hat{Y}_{s n}\right) \hat{X}^{k l} \hat{X}_{; i}^{j m} \hat{X}_{; l}^{n s}+\frac{1}{2} \hat{Y}_{m l} \hat{X}_{; n}^{k m} \hat{X}_{; i}^{n l}\right\} .
\end{aligned}
$$

Symbol “;” in (30), (31) and in what follows stands for the covariant derivatives in background space. The density of gauged gravitational Lagrangian is represented in a form which is characteristic for the theory of quantum fields in the classical background spacetime:

$$
\begin{aligned}
& S=\int \mathrm{d}^{4} x \sqrt{-g}\left(\mathcal{L}_{\text {grav }}-\sqrt{\hat{d}} \Lambda-\frac{1}{4 \varkappa} \hat{X}^{i k} \bar{\theta}_{, i} \theta_{, k}\right), \\
& \mathcal{L}_{\text {grav }}=-\frac{1}{2 \varkappa} \hat{X}^{i k} R_{i k} \\
& +\frac{1}{8 \varkappa}\left[\hat{X}^{k l}\left(\hat{Y}_{j n} \hat{Y}_{s m}-\frac{1}{2} \hat{Y}_{j m} \hat{Y}_{s n}\right) \hat{X}_{; k}^{j m} \hat{X}_{; l}^{s n}-2 \hat{Y}_{i k} \hat{X}_{; m}^{i l} \hat{X}_{; l}^{k m}\right]
\end{aligned}
$$

When the expression for $\mathcal{L}_{\text {grav }}$ was obtained from contraction of tensor (31), the full covariant divergence in the background space have been excluded. Formulas (31), (32) apply for at any parameterization.

Let us discuss the variation method. In the exact quantum theory of gravity with the trivial measure (12), the variation of the action over variables $\hat{\Psi}_{i}^{k}$ leads to the Einstein equations in mixed indexes (15) and (16). In the exact theory, the exponential parameterization is convenient, but, generally speaking, is not necessary. A principally different situation takes place in the approximate self-consistent theory of gravitons in the macroscopic spacetime. In that theory the number of variables doubles, and with this, the classical and quantum components of gravitational fields have to have the status of the dy- namically independent variables due to the doubling of the number of equations. The variation should be done separately over each type of variables. The formalism of the path integration suggests a rigid criterion of dynamic independence: the full measure of integration, by definition, must be factorized with respect to the dynamically independent variables. Obviously, only the exponential parameterization (27), leading to the factorized measure (23), meets the criterion.

The variation of the action over the classic variables is done together with the operation of averaging over the quantum ensemble. In the result, equations for metric of the macroscopic spacetime are obtained:

$$
\begin{aligned}
& \left\langle\Psi\left|\frac{\delta S}{\delta g^{i n}}\right| \Psi\right\rangle \\
& =-2 \varkappa \sqrt{-g} g_{n k}\left\langle\Psi\left|\hat{G}_{i}^{k}-\frac{1}{2} \delta_{i}^{k} \hat{G}_{l}^{l}\right| \Psi\right\rangle=0,
\end{aligned}
$$

where $\hat{G}_{i}^{k}=\hat{\mathcal{G}}_{i}^{k} / \sqrt{-g}$. Variation of the action over background variables, defined as $\Phi_{i}^{k}=\left\langle\Psi\left|\hat{\Psi}_{i}^{k}\right| \Psi\right\rangle$, yields the equations:

$$
\left\langle\Psi\left|\frac{\delta S}{\delta \Phi_{k}^{i}}\right| \Psi\right\rangle=-2 \varkappa \sqrt{-g}\left\langle\Psi\left|\hat{G}_{i}^{k}\right| \Psi\right\rangle=0 .
$$

Equations (33) and (34) are mathematically identical. We should also mention that if the variations over the background metric are done with the fixed mixed components of the quantum field, these equations are valid for any parameterization.

Exponential parameterization (27) has a unique property: the variations over classic $\Phi_{i}^{k}$ (before averaging) and quantum $\hat{\psi}_{i}^{k}$ (without averaging) variables lead to the same equations. That fact is a direct consequence of the relations, showing that variations $\delta \Phi_{l}^{k}$ and $\delta \hat{\psi}_{l}^{k}$ are multiplied by the same operator multiplier:

$$
\begin{array}{lll}
\delta \sqrt{-\hat{g}} \hat{g}^{i k}=\sqrt{-\hat{g}} \hat{g}^{i l} \delta \Phi_{l}^{k}, & & \hat{\psi}_{i}^{k}=\text { const, } \\
\delta \sqrt{-\hat{g}} \hat{g}^{i k}=\sqrt{-\hat{g}} \hat{g}^{i l} \delta \hat{\psi}_{l}^{k}, & \Phi_{i}^{k}=\text { const. }
\end{array}
$$

By a simple operation of subtraction, the identity allows the extraction of pure background terms from the equation of quantum field. The equations of graviton theory in the macroscopic space with self-consistent geometry are written as follows:

$$
\begin{aligned}
& \left\langle\Psi\left|\hat{E}_{i}^{k}\right| \Psi\right\rangle \equiv\left\langle\Psi\left|\hat{G}_{i}^{k}-\frac{1}{2} \delta_{i}^{k} \hat{G}_{l}^{l}\right| \Psi\right\rangle=0, \\
& \hat{L}_{i}^{k} \equiv \hat{G}_{i}^{k}-\frac{1}{2} \delta_{i}^{k} \hat{G}_{l}^{l}-\left\langle\Psi\left|\hat{G}_{i}^{k}-\frac{1}{2} \delta_{i}^{k} \hat{G}_{l}^{l}\right| \Psi\right\rangle=0 .
\end{aligned}
$$

With the exponential parameterization, the formalism of the theory can be expressed in an elegant form. Let us go to the rules of differentiation of exponential matrix functions

$$
\hat{Y}_{i m} \hat{X}_{; l}^{m k}=\hat{\psi}_{i ; l}^{k}, \quad \hat{X}_{; l}^{i k}=\hat{X}^{i m} \hat{\psi}_{m ; l}^{k} .
$$

Taking into account (37), we get the quantum con- 
tribution to the full connectivity (30) as follows

$$
\begin{aligned}
\hat{\mathcal{T}}_{i k}^{l} & =\frac{1}{2}\left(-\hat{\psi}_{i ; k}^{l}-\hat{\psi}_{k ; i}^{l}+\hat{Y}_{k n} \hat{X}^{l m} \hat{\psi}_{i ; m}^{n}\right) \\
& +\frac{1}{4}\left(\delta_{i}^{l} \hat{\psi}_{; k}+\delta_{k}^{l} \hat{\psi}_{; i}-\hat{Y}_{i k} \hat{X}^{l m} \hat{\psi}_{; m}\right) .
\end{aligned}
$$

Formulas (32) could be rewritten as follows:

$$
\begin{aligned}
& \hat{X}_{k}^{l}=(\exp \hat{\psi})_{k}^{l}, \quad \sqrt{\hat{d}}=\mathrm{e}^{\hat{\psi} / 2}, \\
& S=\int \mathrm{d}^{4} x \sqrt{-g}\left(\mathcal{L}_{\text {grav }}-\Lambda \mathrm{e}^{\hat{\psi} / 2}-\frac{1}{4 \varkappa} \hat{X}_{k}^{l} \bar{\theta}^{; k} \theta_{; l}\right), \\
& \mathcal{L}_{\text {grav }}=-\frac{1}{2 \varkappa} \hat{X}_{k}^{l} R_{l}^{k} \\
& +\frac{1}{8 \varkappa} \hat{X}_{k}^{l}\left(\hat{\psi}_{n}^{m ; k} \hat{\psi}_{m ; l}^{n}-\frac{1}{2} \hat{\psi}^{; k} \hat{\psi}_{; l}-2 \hat{\psi}_{n}^{k ; m} \hat{\psi}_{m ; l}^{n}\right) .
\end{aligned}
$$

As is seen from (39), for the exponential parameterization, the non-polynomial structures of quantum theory of gravity have been completely reduced to the factorized exponents ${ }^{3}$.

The explicit form of the tensor, in the terms of which the self-consistent system of equations could be written is as follows

$$
\begin{aligned}
\hat{E}_{i}^{k} \equiv \hat{G}_{i}^{k}-\frac{1}{2} \delta_{i}^{k} \hat{G}_{l}^{l}=\hat{X}^{k l} R_{l i}-\frac{1}{2} \delta_{i}^{k} \hat{X}^{l m} R_{m l}-\delta_{i}^{k} \varkappa \Lambda \mathrm{e}^{\hat{\psi} / 2} \\
+\frac{1}{2}\left[\hat{X}^{l m}\left(\hat{\psi}_{i ; m}^{k}-\hat{\psi}_{m ; i}^{k}\right)-\hat{X}^{k m} \hat{\psi}_{i ; m}^{l}\right. \\
\left.\quad+\frac{1}{2} \delta_{i}^{k}\left(\hat{X}^{m n} \hat{\psi}_{n ; m}^{l}+\hat{X}^{l m} \hat{\psi}_{m ; n}^{n}\right)\right]_{; l} \\
-\frac{1}{4} \hat{X}^{k l}\left(\hat{\psi}_{m ; i}^{n} \hat{\psi}_{n ; l}^{m}-\frac{1}{2} \hat{\psi}_{; i} \hat{\psi}_{; l}-2 \hat{\psi}_{l ; m}^{n} \hat{\psi}_{n ; i}^{m}\right) \\
+\frac{1}{8} \delta_{i}^{k} \hat{X}^{r l}\left(\hat{\psi}_{m ; r}^{n} \hat{\psi}_{n ; l}^{m}-\frac{1}{2} \hat{\psi}_{; r} \hat{\psi}_{; l}-2 \hat{\psi}_{l ; m}^{n} \hat{\psi}_{n ; r}^{m}\right) \\
-\frac{1}{4}\left[\hat{X}^{k l}\left(\bar{\theta}_{; l} \theta_{; i}+\bar{\theta}_{; i} \theta_{; l}\right)-\delta_{i}^{k} \hat{X}^{m l} \bar{\theta}_{; m} \theta_{; l}\right] .
\end{aligned}
$$

${ }^{3}$ We are using the standard definitions. Matrix functions are defined by their expansion into power series as any operator functions:

$$
\hat{U}(\hat{V})=\sum_{n} c_{n} \hat{V}^{n}
$$

The derivative of $n$-th degrees of matrix by the same matrix is defined as

$$
\frac{\partial \hat{V}^{n}}{\partial \hat{V}}=n \hat{V}^{n-1} .
$$

The derivative by numerical (non matrix) parameter $z$ is

$$
\frac{\partial \hat{V}^{n}}{\partial z}=n \hat{V}^{n-1} \cdot \frac{\partial \hat{V}}{\partial z} .
$$

If matrix function $\hat{U}(\hat{V})$ and its derivative $\hat{W}=\partial \hat{U}^{n} / \partial \hat{V}$ are elementary functions, then

$$
\frac{\partial \hat{U}}{\partial z}=\hat{W} \cdot \frac{\partial \hat{V}}{\partial z}
$$

Formulas (37)-(39) are the consequence of these definitions. It worth to mention, that in matrix analysis in all intermediate formulas one should be careful with the index ordering.
Let us introduce the following notations:

$$
\begin{aligned}
& \hat{X}_{(1)}^{i k}=\hat{X}^{i k}-g^{i k}=\hat{\psi}^{i k}+\frac{1}{2} \hat{\psi}^{i l} \hat{\psi}_{l}^{k}+\cdots, \\
& \hat{X}_{(2)}^{i k}=\hat{X}^{i k}-g^{i k}-\hat{\psi}^{i k}=\frac{1}{2} \hat{\psi}^{i l} \hat{\psi}_{l}^{k}+\cdots .
\end{aligned}
$$

With use of (41), let us extract from (40) the terms not containing the quantum field, and the terms linear over the quantum field:

$$
\begin{aligned}
& \hat{E}_{i}^{k}=R_{i}^{k}-\frac{1}{2} \delta_{i}^{k} R-\delta_{i}^{k} \varkappa \Lambda \\
& +\frac{1}{2}\left(\hat{\psi}_{i ; l}^{k ; l}-\hat{\psi}_{l ; i}^{k ; l}-\hat{\psi}_{i ; l}^{l ; k}+\delta_{i}^{k} \hat{\psi}_{m ; l}^{l ; m}\right) \\
& +\hat{\psi}_{l}^{k} R_{i}^{l}-\frac{1}{2} \delta_{i}^{k} \hat{\psi}_{l}^{m} R_{m}^{l}-\frac{1}{2} \delta_{i}^{k} \varkappa \Lambda \hat{\psi}-\varkappa \hat{T}_{i}^{k}, \\
& \hat{T}_{i}^{k}=\hat{T}_{i(\text { grav })}^{k}+\hat{T}_{i(\text { ghost })}^{k},
\end{aligned}
$$

where

$$
\begin{aligned}
& \varkappa \hat{T}_{i(\text { grav }}^{k} \\
= & \frac{1}{4} \hat{X}^{k l}\left(\hat{\psi}_{m ; i}^{n} \hat{\psi}_{n ; l}^{m}-\frac{1}{2} \hat{\psi}_{; i} \hat{\psi}_{; l}-2 \hat{\psi}_{l ; m}^{n} \hat{\psi}_{n ; i}^{m}\right) \\
& -\frac{1}{8} \delta_{i}^{k} \hat{X}^{r l}\left(\hat{\psi}_{m ; r}^{n} \hat{\psi}_{n ; l}^{m}-\frac{1}{2} \hat{\psi}_{; r} \hat{\psi}_{; l}-2 \hat{\psi}_{l ; m}^{n} \hat{\psi}_{n ; r}^{m}\right) \\
& -\frac{1}{2}\left[\hat{X}_{(1)}^{l m}\left(\hat{\psi}_{i ; m}^{k}-\hat{\psi}_{m ; i}^{k}\right)-\hat{X}_{(1)}^{k m} \hat{\psi}_{i ; m}^{l}\right. \\
& \left.+\frac{1}{2} \delta_{i}^{k}\left(\hat{X}_{(1)}^{m n} \hat{\psi}_{n ; m}^{l}+\hat{X}_{(1)}^{l m} \hat{\psi}_{m ; n}^{n}\right)\right] \\
- & \hat{X}_{(2)}^{k l} R_{l i}+\frac{1}{2} \delta_{i}^{k} \hat{X}_{(2)}^{l m} R_{m l}+\delta_{i}^{k} \varkappa \Lambda\left(\mathrm{e}^{\hat{\psi} / 2}-1-\frac{1}{2} \hat{\psi}\right)
\end{aligned}
$$

is the EMT of gravitons;

$$
\varkappa \hat{T}_{i(\text { ghost })}^{k}=-\frac{1}{4}\left[\hat{X}^{k l}\left(\bar{\theta}_{; l} \theta_{; i}+\bar{\theta}_{; i} \theta_{; l}\right)-\delta_{i}^{k} \hat{X}^{m l} \bar{\theta}_{; m} \theta_{; l}\right]
$$

is the EMT of ghosts. In the averaging of (42), it was taken into account that $\left\langle\Psi\left|\hat{\psi}_{i}^{k}\right| \Psi\right\rangle \equiv 0$ by definition of the quantum field. Averaged equations for the classic fields (35) take form of the standard Einstein equations containing averaged EMT of gravitons, renormalized by ghosts:

$$
\begin{aligned}
& \left\langle\Psi\left|\hat{E}_{i}^{k}\right| \Psi\right\rangle \\
& \equiv R_{i}^{k}-\frac{1}{2} \delta_{i}^{k} R-\delta_{i}^{k} \varkappa \Lambda-\varkappa\left\langle\Psi\left|\hat{T}_{i}^{k}\right| \Psi\right\rangle=0 .
\end{aligned}
$$

Quantum dynamic equations for gravitons (36) could be rewritten as follows:

$$
\begin{aligned}
\hat{L}_{i}^{k} & \equiv \frac{1}{2}\left(\hat{\psi}_{i ; l}^{k ; l}-\hat{\psi}_{l ; i}^{k ; l}-\hat{\psi}_{i ; l}^{l ; k}+\delta_{i}^{k} \hat{\psi}_{m ; l}^{l ; m}\right) \\
& -\frac{1}{2} \delta_{i}^{k} \varkappa \Lambda \hat{\psi}+\hat{\psi}_{l}^{k} R_{i}^{l}-\frac{1}{2} \delta_{i}^{k} \hat{\psi}_{l}^{m} R_{m}^{l} \\
& -\varkappa\left(\hat{T}_{i}^{k}-\left\langle\Psi\left|\hat{T}_{i}^{k}\right| \Psi\right\rangle\right)=0 .
\end{aligned}
$$


As is seen in the Equations (46), in the theory of gravitons all nonlinear effects are in the difference between the EMT operator and its average value. System of Equations (45), (46) is closed by the quantum dynamic equations for ghosts, which could be also written in $4 \mathrm{D}$ covariant form:

$$
\left(\hat{X}^{i k} \theta_{; k}\right)_{; i}=0, \quad\left(\hat{X}^{i k} \bar{\theta}_{; k}\right)_{; i}=0
$$

Equations (47) provide the realization of the conservative nature of the ghosts' EMT:

$$
\left\langle\Psi\left|\hat{T}_{i(\text { ghost })}^{k}\right| \Psi\right\rangle_{; k}=0 .
$$

\subsection{Differential Identities}

In the exact theory, which is dealing with the full metric, there is an identity:

$$
\begin{aligned}
\hat{D}_{k}\left\{\hat{g}^{k l} \hat{R}_{l i}-\frac{1}{2} \delta_{i}^{k} \hat{g}^{m l} \hat{R}_{l m}-\delta_{i}^{k} \varkappa \Lambda\right. \\
\left.\quad+\frac{1}{4}\left[\hat{g}^{k l}\left(\bar{\theta}_{; l} \theta_{; i}+\bar{\theta}_{; i} \theta_{; l}\right)-\delta_{i}^{k} \hat{g}^{m l} \bar{\theta}_{; m} \theta_{; l}\right]\right\}=0
\end{aligned}
$$

where $\hat{D}_{k}$ is the covariant derivative in the space with metric $\hat{g}_{i k}$. This identity is satisfied by Bianchi identity and by the ghost equations of motion. In terms of covariant derivative in the background space, identity (49) could be rewritten as follows:

$$
\begin{aligned}
& \hat{E}_{i ; k}^{k}-\frac{1}{2}(\ln \hat{d})_{; k} \hat{E}_{i}^{k}+\hat{\mathcal{T}}_{k l}^{k} \hat{E}_{i}^{l}-\hat{\mathcal{T}}_{i k}^{l} \hat{E}_{l}^{k} \\
& \equiv \hat{E}_{i ; k}^{k}-\hat{\mathcal{T}}_{i k}^{l} \hat{E}_{l}^{k}=0 .
\end{aligned}
$$

For the exponential parameterization, taking into account (38), the expression (50) can be transformed to the following form

$$
\hat{E}_{i ; k}^{k}+\frac{1}{2} \hat{\psi}_{k ; i}^{l}\left(\hat{E}_{l}^{k}-\frac{1}{2} \delta_{l}^{k} \hat{E}_{l}^{l}\right)=0 .
$$

Identity transformation $\hat{E}_{i}^{k} \equiv\left\langle\Psi\left|\hat{E}_{i}^{k}\right| \Psi\right\rangle+\hat{L}_{i}^{k}$ and the subsequent averaging of (51) yields:

$$
\left\langle\Psi\left|\hat{E}_{i}^{k}\right| \Psi\right\rangle_{; k}+\frac{1}{2}\left\langle\Psi\left|\hat{\psi}_{k ; i}^{l}\left(\hat{L}_{l}^{k}-\frac{1}{2} \delta_{l}^{k} \hat{L}_{m}^{m}\right)\right| \Psi\right\rangle=0 .
$$

Here we have used explicitly the fact that $\left\langle\Psi\left|\hat{\psi}_{i}^{k}\right| \Psi\right\rangle \equiv 0,\left\langle\Psi\left|\hat{L}_{i}^{k}\right| \Psi\right\rangle \equiv 0$, by definition. Next, Expression (45) is substituted into (52). Taking into account the Bianchi identity and the conservation of the ghost EMT, we obtain:

$$
\left\langle\Psi\left|\hat{T}_{i(\text { grav })}^{k}\right| \Psi\right\rangle_{; k}=\frac{1}{2}\left\langle\Psi\left|\hat{\psi}_{k ; i}^{l}\left(\hat{L}_{l}^{k}-\frac{1}{2} \delta_{l}^{k} \hat{L}_{m}^{m}\right)\right| \Psi\right\rangle .
$$

As is seen from (53), quantum equations of motion (46) provide the conservation of the averaged EMT of gravitons:

$$
\left\langle\Psi\left|\hat{T}_{i(\text { grav })}^{k}\right| \Psi\right\rangle_{; k}=0 .
$$

Take notice, that tensors $\hat{E}_{i}^{k}$ and $\hat{L}_{i}^{k}$ in (51), (53) are multiplied by the linear forms of graviton field operators only. Such a structure of identities is only valid for the exponential parameterization. This fact is of key value for the computations in the framework of perturbation theory. The order $n$ of the perturbation theory is defined by the highest degree of the field operator in the quantum dynamic equations for gravitons (46). The EMT of gravitons which is consistent with the quantum equation of order $n$ contains averaged products of field operators of the order $n+1$ (e.g., the quadratic EMT is consistent with the linear operator equation). We see that by defining the order of the perturbation theory, we have identity (53), in which all terms are of the same maximal order of the quantum field amplitude:

$$
\begin{aligned}
& \left\langle\Psi\left|\hat{T}_{i(\text { grav })}^{k(n+1)}\right| \Psi\right\rangle_{; k} \\
& =\frac{1}{2}\left\langle\Psi\left|\hat{\psi}_{k ; i}^{l}\left(\hat{L}_{l}^{k(n)}-\frac{1}{2} \delta_{l}^{k} \hat{L}_{m}^{m(n)}\right)\right| \Psi\right\rangle .
\end{aligned}
$$

Such a structure of the identity automatically provides the conservation condition (54) at any order of perturbation theory ${ }^{4}$.

\subsection{One-Loop Approximation}

In the framework of one-loop approximation, quantum fields interact only with the classic gravitational field. Accordingly, Equation (46) are being converted into linear operator equations:

$$
\begin{aligned}
\hat{L}_{i}^{k} & =\frac{1}{2}\left(\hat{\psi}_{i ; l}^{k ; l}-\hat{\psi}_{i ; l}^{l ; k}-\hat{\psi}_{l ; i}^{k ; l}+\delta_{i}^{k} \hat{\psi}_{l ; m}^{m ; l}\right)+\hat{\psi}_{l}^{k} R_{i}^{l} \\
& -\frac{1}{2} \delta_{i}^{k} \hat{\psi}_{m}^{l} R_{l}^{m}-\frac{1}{2} \delta_{i}^{k} \varkappa \Lambda \hat{\psi}=0 .
\end{aligned}
$$

Of course, these equations are separated into the equations of constraints (initial conditions):

$$
\hat{L}_{0}^{0}|\Psi\rangle=0, \quad \hat{L}_{0}^{\alpha}|\Psi\rangle=0, \quad \hat{L}_{\alpha}^{0}|\Psi\rangle=0,
$$

and the equations of motion:

$$
\hat{L}_{\alpha}^{\beta}-\frac{1}{2} \delta_{\alpha}^{\beta} \hat{L}_{l}^{l}=0 .
$$

The equations for ghosts (47) are also transformed into

${ }^{4}$ In the framework of the perturbation theory, any parameterization, except the exponential one, creates mathematically contradictory models, in which the perturbative EMT of gravitons $\left\langle\Psi\left|\hat{T}_{i(\text { grav })}^{k(n+1)}\right| \Psi\right\rangle$ is not conserved. In our opinion, a discussion of artificial methods of solutions of this problem, appeared, for example, if linear parameterization $g_{i k}=g_{i k}+\hat{\psi}_{i k}$ is used, makes no sense. The algorithm we have suggested here is well defined because it is based on the exact procedure of separation between the classical and quantum variables in terms of normal coordinates. We believe there is no other mathematically noncontradictive scheme. 
the linear operator equations:

$$
\theta_{; i}^{; i}=0, \quad \bar{\theta}_{; i}^{; i}=0 .
$$

In the one-loop approximation, the state vector is represented as a product of normalized state vectors of gravitons and ghosts:

$$
|\Psi\rangle=\left|\Psi_{g}\right\rangle\left|\Psi_{g h}\right\rangle .
$$

Equations for macroscopic metric (45) take the form:

$$
\begin{aligned}
& R_{i}^{k}-\frac{1}{2} \delta_{i}^{k} R-\varkappa \delta_{i}^{k} \Lambda \\
& =\varkappa\left(\left\langle\Psi_{g}\left|\hat{T}_{i(\text { grav })}^{k}\right| \Psi_{g}\right\rangle+\left\langle\Psi_{g h}\left|\hat{T}_{i(\text { ghost })}^{k}\right| \Psi_{g h}\right\rangle\right) .
\end{aligned}
$$

The averaged EMTs of gravitons and ghosts in Equation (61) are the quadratic forms of the quantum fields. Assuming that $\hat{X}^{i k}=g^{i k}, \hat{X}_{(1)}^{i k}=\hat{\psi}^{i k}, \hat{X}_{(2)}^{i k}=\hat{\psi}^{i l} \hat{\psi}_{l}^{k} / 2$ in (43), (44), we obtain:

$$
\begin{aligned}
& \hat{T}_{i(\text { grav })}^{k} \\
= & \frac{1}{4 \varkappa}\left\{\hat{\psi}_{m ; i}^{l} \hat{\psi}_{l}^{m ; k}-\frac{1}{2} \hat{\psi}_{; i} \hat{\psi}^{; k}-\hat{\psi}_{i ; m}^{l} \hat{\psi}_{l}^{m ; k}-\hat{\psi}_{l}^{k ; m} \hat{\psi}_{m ; i}^{l}\right. \\
& -\frac{1}{2} \delta_{i}^{k}\left(\hat{\psi}_{m ; n}^{l} \hat{\psi}_{l}^{m ; n}-\frac{1}{2} \hat{\psi}_{; n} \hat{\psi}^{; n}-2 \hat{\psi}_{n ; m}^{l} \hat{\psi}_{l}^{m ; n}\right) \\
& -2\left[\hat{\psi}_{m}^{l} \hat{\psi}_{i}^{k ; m}-\hat{\psi}_{m}^{k} \hat{\psi}_{i}^{l ; m}-\hat{\psi}^{l m} \hat{\psi}_{m ; i}^{k}+\frac{1}{2} \delta_{i}^{k}\left(\hat{\psi}_{m}^{n} \hat{\psi}_{n}^{l}\right)^{; m}\right] ; \\
& \left.-2 \hat{\psi}_{m}^{k} \hat{\psi}_{l}^{m} R_{i}^{l}+\delta_{i}^{k} \hat{\psi}_{l}^{n} \hat{\psi}_{n}^{m} R_{m}^{l}+\frac{1}{2} \delta_{i}^{k} \varkappa \Lambda \hat{\psi}^{2}\right\}, \\
& \hat{T}_{i(\text { ghost }}^{k}=-\frac{1}{4 \varkappa}\left(\bar{\theta}_{; i} \theta^{; k}+\bar{\theta}^{; k} \theta_{; i}-\delta_{i}^{k} \bar{\theta}^{; l} \theta_{; l}\right) .
\end{aligned}
$$

Quantum Equations (56), (59) provide the conservation of tensors (62), (63) in the background space:

$$
\begin{aligned}
& \left\langle\Psi_{g}\left|\hat{T}_{i(\text { grav })}^{k}\right| \Psi_{g}\right\rangle_{; k}=0, \\
& \left\langle\Psi_{g h}\left|\hat{T}_{i(\text { ghost })}^{k}\right| \Psi_{g h}\right\rangle_{; k}=0 .
\end{aligned}
$$

The ghost sector of the theory (56)-(64) corresponds to the gauge (26). Note, however, that all equations of the theory, except gauges, are formally general covariant in the background space. That provides a way of expanding the class of gauges for classic fields. Obviously, we can move from the initial 4-coordinates, corresponding to the classic sector of gauges (26), to any other coordinates, conserving quantum gauge condition

$$
\hat{\psi}_{0}^{i}|\Psi\rangle=0 \text {. }
$$

It is not difficult to see, that in the classic sector any gauges of synchronous type are allowed:

$$
g_{00}=N^{2}(t), \quad g_{0 \alpha}=0 .
$$

where $N(t)$ is an arbitrary function of time.
An important technical detail is that in the perturbation theory the graviton field should be consistent with an additional identity. In one-loop approximation that identity is obtained from the covariant differentiation of Equation (56):

$$
\hat{Q}_{i} \equiv\left(R_{l}^{k}+\varkappa \Lambda \delta_{l}^{k}\right) \hat{\psi}_{k ; i}^{l}=0 .
$$

The appearance of conditions (67) reflects the fact that we are dealing with an approximate theory. As it was already mentioned in Section 2.3, the partition of the metric into classic and quantum components, and, respectively, the factorization of the path integral, can be only done under the condition that additional constrains are applied to the geometry of background space. These constrains are manifested through the structure of the Ricci tensor of the background space which should provide the identity (67) for the solutions of dynamic equations for gravitons. In the Heisenberg form of quantum theory the additional identity can be written as conditions on the state vector:

$$
\hat{Q}_{i}|\Psi\rangle \equiv\left(R_{l}^{k}+\varkappa \Lambda \delta_{l}^{k}\right) \hat{\psi}_{k ; i}^{l}|\Psi\rangle=0
$$

Status of all constrains for the state vectors are the same and are as follows. If (57), (65), (68) exist at the initial moment of time, the internal properties of the theory should provide their existence at any following instance of time.

While one is conducting a concrete one-loop calculation, there is a problem of gauge invariance of the total EMT of gravitons and ghosts. As was mentioned by De Witt [17], after the separation of the metric into background and graviton components, the transformations of the diffeomorphism group (4) can be represented as transformations of the internal gauge symmetry of graviton field. In the framework of one-loop approximation, these transformations are as follows:

$$
\delta \hat{\psi}_{i}^{k}=-\delta_{i}^{k} \eta_{; l}^{l}+\eta_{; i}^{k}+\eta_{i}^{; k} .
$$

The problem of gauge non-invariance is twofold. First, the EMT of gravitons (62) is not invariant with respect to transformations in (69). Second, the ghost sector (the ghost EMT), inevitably presented in the theory, depends on the gauge. Concerning the first problem, it is known that the operation removing gauge non-invariant terms from the EMT of gravitons belongs to the operation of averaging over a quantum ensemble. In the general case of arbitrary background geometry and arbitrary graviton wavelengths we encounter a number of problems (when conducting this operation), which should be discussed separately.

In the particular case of the theory of gravitons in a homogeneous and isotropic Universe, the averaging problem has a consistent mathematical solution. It was shown in Section 3.1 that removing the gauge non- 
invariant contributions from the EMT of gravitons from the quantum ensemble has been set gauge-invariantly. To address the second aspect of the problem, we should take into account that the theory of gravitons in the macroscopic space with the self-consistent geometry operates with macroscopic observables. Therefore, in this theory one-loop finiteness, as the general property of one-loop quantum gravity, should have a specific embodiment: by their mathematical definition, macroscopic observables must be the finite values. This requirement on the theory in the Heisenberg representation is realized in Hamilton gauge (26) only.

\section{Self-Consistent Theory of Gravitons in the Isotropic Universe}

\subsection{Elimination of 3-Vector and 3-Scalar Modes by Conditions Imposed on the State Vector}

We consider the quantum theory of gravitons in the spacetime with the following background metric

$$
\begin{aligned}
d s^{2} & =g_{i k} d x^{i} d x^{k} \\
& =N^{2}(t) d t^{2}-a^{2}(t)\left(d x^{2}+d y^{2}+d z^{2}\right) .
\end{aligned}
$$

In this space the graviton field is expanded over the irreducible representations of the group of three-dimensional rotations, i.e. over 3-tensor $\hat{\psi}_{\alpha(t)}^{\beta}$, 3-vector

$\hat{\psi}_{i(v)}^{k}=\left(\hat{\psi}_{0(v)}^{\alpha}, \hat{\psi}_{\alpha(v)}^{\beta}\right)$ and 3-scalar

$\hat{\psi}_{i(s)}^{k}=\left(\hat{\psi}_{0(s)}^{0}, \hat{\psi}_{0(s)}^{\alpha}, \hat{\psi}_{\alpha(s)}^{\beta}\right)$ modes. Equations (56) are split

into three independent systems of equations, so that each of such systems represents each mode separately. The state vector of gravitons is of multiplicative form that reads

$$
\left|\Psi_{g}\right\rangle=\left|\Psi_{t}\right\rangle\left|\Psi_{v}\right\rangle\left|\Psi_{s}\right\rangle .
$$

The averaged EMT (60) is presented by an additive form that reads:

$$
\begin{aligned}
& \left\langle\Psi_{g}\left|\hat{T}_{i(\text { grav })}^{k}\right| \Psi_{g}\right\rangle \\
& =\left\langle\Psi_{t}\left|\hat{T}_{i(t)}^{k}\right| \Psi_{t}\right\rangle+\left\langle\Psi_{v}\left|\hat{T}_{i(v)}^{k}\right| \Psi_{v}\right\rangle+\left\langle\Psi_{s}\left|\hat{T}_{i(s)}^{k}\right| \Psi_{s}\right\rangle .
\end{aligned}
$$

The averaged EMT contains no products of modes that belong to different irreducible representations. This is because the equality $\left\langle\Psi_{g}\left|\hat{\psi}_{i}^{k}\right| \Psi_{g}\right\rangle=0$ is divided into three following three independent equalities

$$
\begin{aligned}
& \left\langle\Psi_{s}\left|\hat{\psi}_{i(s)}^{k}\right| \Psi_{s}\right\rangle=0, \quad\left\langle\Psi_{v}\left|\hat{\psi}_{i(v)}^{k}\right| \Psi_{v}\right\rangle=0, \\
& \left\langle\Psi_{t}\left|\hat{\psi}_{i(t)}^{k}\right| \Psi_{t}\right\rangle=0 .
\end{aligned}
$$

Equalities (72) are conditions that provide the consistency of properties of quantum ensemble of gravitons with the properties of homogeneity and isotropy of the background. In the homogeneous and isotropic space, the same equalities hold for Fourier images of the graviton field. Therefore, the satisfaction of these equalities is provided by the isotropy of graviton spectrum in the $\boldsymbol{k}$ space and by the equivalence of different polarizations.

3-tensor modes $\hat{\psi}_{\alpha(t)}^{\beta}$ and their EMT $\left\langle\Psi_{t}\left|\hat{T}_{i(t)}^{k}\right| \Psi_{t}\right\rangle$, respectively, are gauge invariant objects. Gauge non-invariant modes $\hat{\psi}_{i(v)}^{k}, \hat{\psi}_{i(s)}^{k}$ are eliminated by conditions that, imposed on the state vector, read

$$
\hat{\psi}_{i(v)}^{k}\left|\Psi_{v}\right\rangle=\hat{\psi}_{i(s)}^{k}\left|\Psi_{s}\right\rangle=0 .
$$

Note that the conditions (73) automatically follow from Equation (56) and conditions (63). As a result of this, a gauge non-invariant EMT of 3-scalar and 3-vector modes is eliminated from the macroscopic Einstein equations, and we get

$$
\left\langle\Psi_{v}\left|\hat{T}_{i(v)}^{k}\right| \Psi_{v}\right\rangle=0, \quad\left\langle\Psi_{s}\left|\hat{T}_{i(s)}^{k}\right| \Psi_{s}\right\rangle=0 .
$$

The important fact is that in the isotropic Universe, the separation of gauge invariant EMT of 3-tensor gravitons is accomplished without the use of short-wave approximation. In connection with this, note the following fact. In the theory, which formally operates with waves of arbitrary lengths, the problem of existence of a quantum ensemble of waves with wavelengths greater than the distance from horizon is open [13]. In cosmology, the existence of such an ensemble is provided by the following experimental fact. In the real Universe (whose properties are controlled by observational data beginning from the instant of recombination), the characteristic scale of casually-connected regions is much greater (many orders of magnitude) than the formal horizon of events. The standard explanation of this fact is based on the hypothesis of early inflation. Taking into account these circumstances, we do not impose any additional restrictions on the quantum ensemble.

The procedure described above is based on the existence of independent irreducible representations of graviton modes only. But in this procedure, gauge-noninvariant modes are eliminated by using of a gauge, i.e. they are eliminated by using of gauge-non-invariant procedures. The gauge-invariant procedure of getting the same results is presented in [24], Section 3.1.

\subsection{Canonical Quantization of 3-Tensor Gravitons and Ghosts}

The parameters of gauge transformations do not contain terms of expansion over transverse 3-tensor plane waves. Therefore, Fourier images of tensor fluctuations are gaugeinvariant by definition. We have

$$
\begin{aligned}
& \hat{\psi}_{0}^{0}(\boldsymbol{k})=0, \quad \hat{\psi}_{\alpha}^{0}(\boldsymbol{k})=-\hat{\psi}_{0}^{\alpha}(\boldsymbol{k})=0, \\
& \hat{\psi}_{\alpha}^{\beta}(\boldsymbol{k} \sigma)=Q_{\alpha}^{\beta}(\boldsymbol{k} \sigma) \hat{\psi}_{\boldsymbol{k} \sigma}, \\
& k_{\alpha} Q_{\beta}^{\alpha}(\boldsymbol{k} \sigma) \equiv 0, \quad Q_{\alpha}^{\alpha}(\boldsymbol{k} \sigma) \equiv 0
\end{aligned}
$$


where $\sigma$ is the index of transverse polarizations. The operator equation for 3-tensor gravitons is

$$
\begin{aligned}
& \psi_{\alpha(t)}^{\beta}(t, \boldsymbol{x})=\sum_{k \sigma} Q_{\alpha}^{\beta}(\boldsymbol{k} \sigma) \psi_{k \sigma}(t) \mathrm{e}^{i \boldsymbol{k} x}, \\
& \ddot{\psi}_{k \sigma}+3 H \dot{\psi}_{\boldsymbol{k} \sigma}+\frac{k^{2}}{a^{2}} \psi_{\boldsymbol{k} \sigma}=0,
\end{aligned}
$$

where $H=\dot{a} / a$ is Hubble function and dots mean derivatives with respect to the physical time $t$.

The special property of the gauge used is the following. The differential equation for ghosts is obtained from the equation for gravitons by exchange of graviton operator with the ghost operator. It reads

$$
\theta(t, \boldsymbol{x})=\sum_{\boldsymbol{k}} \theta_{\boldsymbol{k}}(t) \mathrm{e}^{i \boldsymbol{k} x}, \quad \ddot{\theta}_{\boldsymbol{k}}+3 H \dot{\theta}_{\boldsymbol{k}}+\frac{k^{2}}{a^{2}} \theta_{\boldsymbol{k}}=0 .
$$

Macroscopic Einstein Equation (61) read

$$
\begin{gathered}
3 H^{2}=\varkappa\left(\varepsilon_{g}+\Lambda\right), \\
2 \dot{H}+3 H^{2}=\varkappa\left(\Lambda-p_{g}\right),
\end{gathered}
$$

where

$$
\begin{aligned}
\varepsilon_{g}= & \frac{1}{8 \varkappa} \sum_{\boldsymbol{k} \sigma}\left\langle\Psi_{g}\left|\dot{\hat{\psi}}_{\boldsymbol{k} \sigma}^{+} \dot{\hat{\psi}}_{\boldsymbol{k} \sigma}+\frac{k^{2}}{a^{2}} \hat{\psi}_{\boldsymbol{k} \sigma}^{+} \hat{\psi}_{\boldsymbol{k} \sigma}\right| \Psi_{g}\right\rangle \\
& -\frac{1}{4 \varkappa} \sum_{\boldsymbol{k}}\left\langle\Psi_{g h}\left|\dot{\bar{\theta}}_{\boldsymbol{k}} \dot{\theta}_{\boldsymbol{k}}+\frac{k^{2}}{a^{2}} \bar{\theta}_{\boldsymbol{k}} \theta_{\boldsymbol{k}}\right| \Psi_{g h}\right\rangle, \\
p_{g}= & \frac{1}{8 \varkappa} \sum_{\boldsymbol{k} \sigma}\left\langle\Psi_{g}\left|\dot{\hat{\psi}}_{\boldsymbol{k} \sigma}^{+} \dot{\hat{\psi}}_{\boldsymbol{k} \sigma}-\frac{k^{2}}{3 a^{2}} \hat{\psi}_{\boldsymbol{k} \sigma}^{+} \hat{\psi}_{\boldsymbol{k} \sigma}\right| \Psi_{g}\right\rangle \\
& -\frac{1}{4 \varkappa} \sum_{\boldsymbol{k}}\left\langle\Psi_{g h}\left|\dot{\bar{\theta}}_{\boldsymbol{k}} \dot{\theta}_{\boldsymbol{k}}-\frac{k^{2}}{3 a^{2}} \bar{\theta}_{\boldsymbol{k}} \theta_{\boldsymbol{k}}\right| \Psi_{g h}\right\rangle
\end{aligned}
$$

are the energy density and pressure of gravitons that are renormalized by ghosts. Formulas (80) were obtained after elimination of 3-scalar and 3-vector modes from Equations (62) and (63). We also took into account the following definitions

$$
\begin{aligned}
& \left\langle\Psi\left|\hat{T}_{0}^{0}\right| \Psi\right\rangle=\varepsilon_{g}, \\
& \left\langle\Psi\left|\hat{T}_{\alpha}^{\beta}\right| \Psi\right\rangle=\frac{\delta_{\alpha}^{\beta}}{3}\left\langle\Psi\left|\hat{T}_{\gamma}^{\gamma}\right| \Psi\right\rangle=-\delta_{\alpha}^{\beta} p_{g} .
\end{aligned}
$$

Also we have the following rules of averaging of bilinear forms that are the consequence of homogeneity and isotropy of the background

$$
\begin{aligned}
& \left\langle\Psi_{g}\left|\hat{\psi}_{\boldsymbol{k} \sigma}^{+} \hat{\psi}_{\boldsymbol{k}^{\prime} \sigma^{\prime}}\right| \Psi_{g}\right\rangle=\left\langle\Psi_{g}\left|\hat{\psi}_{\boldsymbol{k} \sigma}^{+} \hat{\psi}_{\boldsymbol{k} \sigma}\right| \Psi_{g}\right\rangle \delta_{\boldsymbol{k} \boldsymbol{k}^{\prime}} \delta_{\sigma \sigma^{\prime}}, \\
& \left\langle\Psi_{g h}\left|\bar{\theta}_{\boldsymbol{k}} \theta_{\boldsymbol{k}^{\prime}}\right| \Psi_{g h}\right\rangle=\left\langle\Psi_{g h}\left|\bar{\theta}_{\boldsymbol{k}} \theta_{\boldsymbol{k}}\right| \Psi_{g h}\right\rangle \delta_{\boldsymbol{k}^{\prime}} .
\end{aligned}
$$

The self-consistent system of Equations (76)-(79) is a particular case of general equations of one-loop quantum gravity (56), (59), (61)-(63). In turn, these general equations are the result of the transition to the one-loop approximation from exact Equations (43)-(47) that were obtained by variation of gauged action over classic and quantum variables. To canonically quantize 3-tensor gravitons and ghosts, one needs to make sure that the variational procedure takes place for Equations (76)-(79) directly. To do so, in the action (39) we keep only background terms and terms that are quadratic over 3-tensor fluctuations and ghosts. Then, we exclude the full derivative from the background sector and make the transition to Fourier images in the quantum sector. As a result of these operations, we obtain the following

$$
\begin{aligned}
& S=\int \mathrm{d} t\left(-\frac{3 \dot{a}^{2} a}{\varkappa N}-\Lambda a^{3} N+L_{\text {grav }}+L_{\text {ghost }}\right), \\
& L_{\text {grav }}+L_{\text {ghost }}= \frac{1}{8 \varkappa} \sum_{\boldsymbol{k} \sigma}\left(\frac{a^{3}}{N} \dot{\hat{\psi}}_{\boldsymbol{k} \sigma}^{+} \dot{\hat{\psi}}_{\boldsymbol{k} \sigma}-N a k^{2} \hat{\psi}_{\boldsymbol{k} \sigma}^{+} \hat{\psi}_{\boldsymbol{k} \sigma}\right) \\
&-\frac{1}{4 \varkappa} \sum_{\boldsymbol{k}}\left(\frac{a^{3}}{N} \dot{\bar{\theta}}_{\boldsymbol{k}} \dot{\theta}_{\boldsymbol{k}}-N a k^{2} \bar{\theta}_{\boldsymbol{k}} \theta_{\boldsymbol{k}}\right) .
\end{aligned}
$$

In (81), the background metric is taken to be in the form of (70), and the $N$ function is taken to be a variation variable (the choice of this function, e.g. $N=1$, to be made after variation of action). Here and further on, the normalized volume is supposed to be unity, so $V=\int \mathrm{d}^{3} x=1$. The terms which are linear over the graviton field are eliminated from (81) because of zero trace of 3-tensor fluctuations. Variations of action over $N$ and $a$ are done with the following averaging. These procedures lead to Equations (78), (79) and Expression (80). Variation of action over quantum variables leads to the quantum equations of motion (76) and (77).

In accordance with the standard procedure of canonical quantization of gravitons, one introduces generalized momenta

$$
\hat{\pi}_{\boldsymbol{k} \sigma}=\frac{\partial L}{\partial \dot{\hat{\psi}}_{\boldsymbol{k} \sigma}}=\frac{a^{3}}{4 \varkappa} \dot{\hat{\psi}}_{\boldsymbol{k} \sigma}^{+} .
$$

Then, commutation relations between operators that are defined at the same instant of time read

$$
\begin{aligned}
{\left[\hat{\pi}_{\boldsymbol{k} \sigma}, \hat{\psi}_{\boldsymbol{k}^{\prime} \sigma^{\prime}}\right]_{-} } & \equiv \frac{a^{3}}{4 \varkappa}\left[\dot{\hat{\psi}}_{\boldsymbol{k} \sigma}^{+}, \hat{\psi}_{\boldsymbol{k}^{\prime} \sigma^{\prime}}\right]_{-} \\
& =-i \hbar \delta_{\boldsymbol{k} \boldsymbol{k}^{\prime}} \delta_{\sigma \sigma^{\prime}} .
\end{aligned}
$$

Formulas (82) and (83) are presented for the $N=1$ case. Note also that the derivative in (82) should be calculated taking into account the $\psi_{\boldsymbol{k} \sigma}^{+}=\psi_{-\boldsymbol{k}-\sigma}$ condition.

The ghost quantization contains three specific issues. First, there is the following technical detail that must be taken into account for the definition of generalized momenta of ghost fields. The argument in respect to which the differentiation is conducted needs to be considered as a left co-multiplier of quadratic form. Executing the appropriate requirement and taking into account Grassman's character of ghost fields, we obtain 


$$
\mathcal{P}_{k}=\frac{\partial L}{\partial \dot{\theta}_{k}}=\frac{a^{3}}{4 \varkappa} \dot{\bar{\theta}}_{k}, \quad \overline{\mathcal{P}}_{k}=\frac{\partial L}{\partial \dot{\bar{\theta}}_{k}}=-\frac{a^{3}}{4 \varkappa} \dot{\theta}_{k} .
$$

Second, the quantization of Grassman's fields is carried out by setting the following anti-commutation relations

$$
\begin{aligned}
& {\left[\mathcal{P}_{\boldsymbol{k}}, \theta_{\boldsymbol{k}}\right]_{+} \equiv \frac{a^{3}}{4 \varkappa}\left[\dot{\bar{\theta}}_{\boldsymbol{k}}, \theta_{\boldsymbol{k}^{\prime}}\right]_{+}=-i \hbar \delta_{\boldsymbol{k} \boldsymbol{k}^{\prime}},} \\
& {\left[\overline{\mathcal{P}}_{\boldsymbol{k}}, \bar{\theta}_{\boldsymbol{k}}\right]_{+} \equiv-\frac{a^{3}}{4 \varkappa}\left[\dot{\theta}_{\boldsymbol{k}}, \bar{\theta}_{\boldsymbol{k}^{\prime}}\right]_{+}=-i \hbar \delta_{\boldsymbol{k k}^{\prime}}}
\end{aligned}
$$

Third is the bosonization of ghost fields, which is carried out after quantization of (85). The possibility of the bosonization procedure is provided by Grassman algebra, which contains Grassman units defined by relations $\bar{u} u=-u \bar{u}=1$. Therefore, conjunctive Grassman fields can be always presented in the following form

$$
\theta_{k}=u \vartheta_{k}, \quad \bar{\theta}_{k}=\bar{u} \vartheta_{k}^{+},
$$

where $\vartheta_{\boldsymbol{k}}$ is Fourier image of complex scalar field which is described by the usual algebra. The substitution of (86) in (85) leads to the following standard Bose commutation relations

$$
\begin{aligned}
& \frac{a^{3}}{4 \varkappa}\left[\dot{\vartheta}_{\boldsymbol{k}}^{+}, \vartheta_{\boldsymbol{k}^{\prime}}\right]_{-}=-i \hbar \delta_{\boldsymbol{k} \boldsymbol{k}^{\prime}}, \\
& \frac{a^{3}}{4 \varkappa}\left[\dot{\vartheta}_{\boldsymbol{k}}, \vartheta_{\boldsymbol{k}^{\prime}}^{+}\right]_{-}=-i \hbar \delta_{\boldsymbol{k} \boldsymbol{k}^{\prime}}
\end{aligned}
$$

The Hermit conjugation transforms one of them to the other.

\subsection{State Vector of the General Form}

To complete the self-consistent theory of gravitons in the isotropic Universe, one needs to present the algorithm of introduction of the graviton-ghost ensemble into the theory. Properties of this ensemble are defined by Heisenberg's state vector which is expanded over the basis that has a physical interpretation. Any possible basis is the system of eigenvectors of an appropriate time independent Hermit operator. The existence of such operators can be proved in a general form. Let us consider the following operator equation which is an analog of operator equations of gravitons and ghosts

$$
\ddot{y}_{k}+3 H \dot{y}_{k}+\frac{k^{2}}{a^{2}} y_{k}=0 \text {. }
$$

Coefficients of Equation (88) are continuous and differentiated functions of time along all cosmological scales except for the singularity. Thus, with the exception of the singular point, the general solution of Equation (88) definitely exists. Below we will show that the existence of a state vector follows only from the existence of general solution of Equation (88) (see also [13]).
Suppose $g_{k}, h_{k}$ are linear independent solutions to (88), so that their superposition with arbitrary coefficients gives the general solution to (88). With no loss of generality, one can suppose that these solutions are normalized in some convenient way in each concrete case. From the theory of ordinary differential equations it is known that $g_{k}, h_{k}$ functions are connected to each other by the following relation

$$
g_{k} \dot{h}_{k}-h_{k} \dot{g}_{k}=\frac{C_{k}}{a^{3}},
$$

where $C_{k}$ is a normalization constant. The comparison of (88) with (76) and (77) shows that solutions of operator equations are presented by the same functions. For operators of graviton field we have

$$
\hat{\psi}_{\boldsymbol{k} \sigma}=\hat{A}_{\boldsymbol{k} \sigma} g_{\boldsymbol{k}}+\hat{B}_{\boldsymbol{k} \sigma} h_{\boldsymbol{k}},
$$

where $\hat{A}_{\boldsymbol{k} \sigma}, \hat{B}_{\boldsymbol{k} \sigma}$ are operator constants of integration. Directly from these operator constants, one needs to build the operator which gives rise to the full set of basis vectors.

It is important to keep in mind that commutation property of operator constants $\hat{A}_{\boldsymbol{k} \sigma}, \hat{B}_{\boldsymbol{k} \sigma}$ and physical interpretation of basis state vectors are determined by the choice of linear independent solutions of Equation (88). The simplest basis is that of occupation numbers. The choice of linear independent solutions as self-conjugated complex functions corresponds to this basis.

In accordance with (89), if $g_{k}=f_{k}, h_{k}=f_{k}^{*}$ the normalization constant is pure imaginary. Let's take $C_{k}=i$, so we obtain

$$
f_{k} \dot{f}_{k}^{*}-f_{k}^{*} \dot{f}_{k}=\frac{i}{a^{3}} .
$$

To build the graviton operator over this basis, one need to carry out the multiplicative renormalization of operator constants taking into account that field is real. This yield

$$
A_{\boldsymbol{k} \sigma}=\sqrt{4 \varkappa \hbar} \hat{c}_{\boldsymbol{k} \sigma}, \quad B_{\boldsymbol{k} \sigma}=\sqrt{4 \varkappa \hbar} \hat{c}_{-\boldsymbol{k}-\sigma}^{+} .
$$

As result of these operations, we get the graviton operator and its derivative that read

$$
\begin{aligned}
& \hat{\psi}_{\boldsymbol{k} \sigma}=\sqrt{4 \varkappa \hbar}\left(\hat{c}_{\boldsymbol{k} \sigma} f_{k}+\hat{c}_{-\boldsymbol{k}-\sigma}^{+} f_{k}^{*}\right), \\
& \dot{\hat{\psi}}_{\boldsymbol{k} \sigma}^{+}=\sqrt{4 \varkappa \hbar}\left(c_{\boldsymbol{k} \sigma}^{+} \dot{f}_{k}^{*}+c_{-\boldsymbol{k}-\sigma} \dot{f}_{k}\right) .
\end{aligned}
$$

Standard commutation relations for operators of graviton creation and annihilation are obtained by the substitution of (92) into (83) and taking into account (91). They read

$$
\begin{aligned}
& {\left[\hat{c}_{\boldsymbol{k} \sigma}, \hat{c}_{\boldsymbol{k}^{\prime} \sigma^{\prime}}^{+}\right]_{-}=\delta_{\boldsymbol{k} \boldsymbol{k}^{\prime}} \delta_{\sigma \sigma^{\prime}},} \\
& {\left[\hat{c}_{\boldsymbol{k} \sigma}, \hat{c}_{\boldsymbol{k}^{\prime} \sigma^{\prime}}\right]_{-}=0, \quad\left[\hat{c}_{\boldsymbol{k} \sigma}^{+}, \hat{c}_{\boldsymbol{k}^{\prime} \sigma^{\prime}}^{+}\right]_{-}=0 .}
\end{aligned}
$$


In accordance with (93), the operator of occupation numbers $\hat{n}_{\boldsymbol{k} \sigma}=\hat{c}_{\boldsymbol{k} \sigma}^{+} \hat{c}_{\boldsymbol{k} \sigma}$ exists that gives rise to basis vectors $\left|n_{k \sigma}\right\rangle$ of Fock's space. Non-negative integer numbers $n_{k \sigma}=0,1,2, \cdots$ are eigenvalues of this operator.

In accordance with (80), the observables are additive over modes with given $\boldsymbol{k} \sigma$. Therefore, the state vector is of multiplicative structure that reads

$$
\left|\Psi_{g}\right\rangle=\prod_{k \sigma}\left|\Psi_{k \sigma}\right\rangle,
$$

where $\left|\Psi_{\boldsymbol{k} \sigma}\right\rangle$ is state vector of $\boldsymbol{k} \sigma$-subsystem of gravitons of momentum $\boldsymbol{p}=\hbar \boldsymbol{k}$ and polarization $\sigma$. In turn, in a general case, $\left|\Psi_{k \sigma}\right\rangle$ is an arbitrary superposition of vectors that corresponds to different occupation numbers but the same $\boldsymbol{k} \sigma$ values. Suppose that $\mathcal{C}_{n_{k \sigma}}$ is the amplitude of probability of finding the $\boldsymbol{k} \sigma$ subsystem of gravitons in the state with the occupation number $n_{k \sigma}$. If so, then the state vector of the general form is the product of normalized superpositions

$$
\left|\Psi_{g}\right\rangle=\prod_{\boldsymbol{k} \sigma n_{\boldsymbol{k} \sigma}} \sum_{n_{\boldsymbol{k} \sigma}}\left|n_{\boldsymbol{k} \sigma}\right\rangle, \quad \sum_{n_{\boldsymbol{k} \sigma}}\left|\mathcal{C}_{n_{\boldsymbol{k} \sigma}}\right|^{2}=1 .
$$

After the bosonization in the ghost sector is done, one gets equations of motion and commutation relations that are similar to those for graviton. The same set of linear independent solutions $f_{k}, f_{k}^{*}$ that was introduced for operators of graviton field is used for operators of ghost fields. What is necessary to take into account here is originally complex character of ghost fields, which leads to $\vartheta_{k}^{+} \neq \vartheta_{-k}$. As a result, operators of ghost and antighosts creation and annihilation appear in the theory. They read

$$
\begin{aligned}
& \vartheta_{\boldsymbol{k}}=\sqrt{4 \varkappa \hbar}\left(\hat{a}_{\boldsymbol{k}} f_{k}+\hat{b}_{-\boldsymbol{k}}^{+} f_{k}^{*}\right), \\
& \dot{\vartheta}_{\boldsymbol{k}}^{+}=\sqrt{4 \varkappa \hbar}\left(a_{\boldsymbol{k}}^{+} \dot{f}_{k}^{*}+b_{-\boldsymbol{k}} \dot{f}_{k}\right) .
\end{aligned}
$$

The substitution of (95) into (87) leads to standard commutation relations

$$
\begin{aligned}
& {\left[\hat{a}_{\boldsymbol{k}}, \hat{a}_{\mathbf{k}^{\prime}}^{+}\right]_{-}=\delta_{\boldsymbol{k}^{\prime}}, \quad\left[\hat{a}_{\boldsymbol{k}}, \hat{a}_{\mathbf{k}^{\prime}}\right]_{-}=\left[\hat{a}_{\mathbf{k}}^{+}, \hat{a}_{\mathbf{k}^{\prime}}^{+}\right]_{-}=0,} \\
& {\left[\hat{b}_{\boldsymbol{k}}, \hat{b}_{\mathbf{k}^{\prime}}^{+}\right]_{-}=\delta_{\boldsymbol{k} \boldsymbol{k}^{\prime}}, \quad\left[\hat{b}_{\boldsymbol{k}}, \hat{b}_{\boldsymbol{k}^{\prime}}\right]_{-}=\left[\hat{b}_{\boldsymbol{k}}^{+}, \hat{b}_{\mathbf{k}^{\prime}}^{+}\right]_{-}=0,} \\
& {\left[\hat{a}_{k}, \hat{b}_{\mathbf{k}^{\prime}}\right]_{-}=\left[\hat{a}_{\boldsymbol{k}}, \hat{b}_{\mathbf{k}^{\prime}}^{+}\right]_{-}=0,\left[\hat{a}_{k}^{+}, \hat{b}_{\mathbf{k}^{\prime}}\right]_{-}=\left[\hat{a}_{k}^{+}, \hat{b}_{\mathbf{k}^{\prime}}^{+}\right]_{-}=0}
\end{aligned}
$$

Applying the reasoning which is similar to that described above, we conclude that in the ghost sector, the state vector of the general form is also given by product of normalized superpositions. It reads

$$
\begin{aligned}
& \left|\Psi_{g h}\right\rangle=\prod_{k} \sum_{n_{k}} \mathcal{A}_{n_{k}}\left|n_{k}\right\rangle \prod_{k} \sum_{\bar{n}_{k}} \mathcal{B}_{\bar{n}_{k}}\left|\bar{n}_{k}\right\rangle, \\
& \sum_{n_{k}}\left|\mathcal{A}_{n_{k}}\right|^{2}=\sum_{\bar{n}_{k}}\left|\mathcal{B}_{\bar{n}_{k}}\right|^{2}=1 .
\end{aligned}
$$

The set of amplitudes $\mathcal{C}_{n_{k \sigma}}, \mathcal{A}_{n_{k}}, \mathcal{B}_{\bar{n}_{k}}$, which para- meterizes Heisenberg's state vector actually determines the initial condition of quantum system of gravitons and ghosts.

Formulas (94) and (97) can be also used in case when real functions are chosen as linear independent solutions of Equation (88). The justification for this is due to the fact that real linear independent solutions can be obtained from complex self-conjugated ones by the following linear transformation

$$
g_{k}=\frac{1}{\sqrt{2}}\left(f_{k}+f_{k}^{*}\right), \quad h_{k}=\frac{i}{\sqrt{2}}\left(f_{k}-f_{k}^{*}\right) .
$$

After transition to the basis of real functions in (92) and (95), we get

$$
\begin{aligned}
& \hat{\psi}_{\boldsymbol{k} \sigma}=\sqrt{4 \varkappa \hbar}\left(\hat{Q}_{\boldsymbol{k} \sigma} g_{k}+\hat{P}_{\boldsymbol{k} \sigma} h_{k}\right), \\
& \hat{\vartheta}_{\boldsymbol{k}}=\sqrt{4 \varkappa \hbar}\left(\hat{q}_{\boldsymbol{k}} g_{k}+\hat{p}_{\boldsymbol{k}} h_{k}\right),
\end{aligned}
$$

where

$$
\begin{aligned}
& \hat{Q}_{\boldsymbol{k} \sigma}=\hat{Q}_{-\boldsymbol{k}-\sigma}^{+}=\frac{1}{\sqrt{2}}\left(\hat{c}_{\boldsymbol{k} \sigma}+c_{-\boldsymbol{k}-\sigma}^{+}\right), \\
& \hat{P}_{\boldsymbol{k} \sigma}=\hat{P}_{-\boldsymbol{k}-\sigma}^{+}=-\frac{i}{\sqrt{2}}\left(\hat{c}_{\boldsymbol{k} \sigma}-c_{-\boldsymbol{k}-\sigma}^{+}\right), \\
& \hat{q}_{\boldsymbol{k}}=\frac{1}{\sqrt{2}}\left(\hat{a}_{\boldsymbol{k}}+b_{-\boldsymbol{k}}^{+}\right), \\
& \hat{p}_{\boldsymbol{k}}=-\frac{i}{\sqrt{2}}\left(\hat{a}_{\boldsymbol{k}}-b_{-\boldsymbol{k}}^{+}\right) .
\end{aligned}
$$

Relations (100) allow to work with real linear independent solutions and to use simultaneously state vectors (94) and (97) for the representation of occupation numbers. Note that in the framework of the basis of real functions, operator constants are operators of generalized coordinates and momenta:

$$
\left[\hat{P}_{k \sigma}^{+}, \hat{Q}_{\boldsymbol{k}^{\prime} \sigma^{\prime}}\right]_{-}=-i \delta_{k k^{\prime}} \delta_{\sigma \sigma^{\prime}}, \quad\left[\hat{p}_{\boldsymbol{k}}^{+}, \hat{q}_{\boldsymbol{k}^{\prime} \sigma^{\prime}}\right]_{-}=-i \delta_{k k^{\prime}} .
$$

To complete this Section, let us discuss two problems that are relevant to intrinsic mathematical properties of the theory. First of all, let us mention that "bosonization" of ghost fields is a necessary element of the theory because only this procedure provides the existence of state vector in the ghost sector. Mathematically, it is because the structure of the classic differential equation (88) and properties of its solution (91) are inconsistent with the Fermi-Dirac quantization. In terms of original ghost fields we have

$$
\begin{aligned}
& \theta_{\boldsymbol{k}}=\sqrt{4 \varkappa \hbar}\left(\alpha_{\boldsymbol{k}} f_{k}+\bar{\beta}_{-\boldsymbol{k}} f_{k}^{*}\right), \\
& \dot{\bar{\theta}}_{\boldsymbol{k}}=\sqrt{4 \varkappa \hbar}\left(\bar{\alpha}_{\boldsymbol{k}} \dot{f}_{k}^{*}+\beta_{-\boldsymbol{k}} \dot{f}_{k}\right) .
\end{aligned}
$$

Substitution (102) into (85) and taking into account (91) leads to anti-commutation relations for operator constants that read 


$$
\left[\bar{\alpha}_{\boldsymbol{k}}, \alpha_{\mathbf{k}^{\prime}}\right]_{+}=-\delta_{\boldsymbol{k} \mathbf{k}^{\prime}}, \quad\left[\beta_{\boldsymbol{k}}, \bar{\beta}_{\boldsymbol{k}^{\prime}}\right]_{+}=\delta_{\boldsymbol{k} \mathbf{k}^{\prime}} .
$$

The $\left[\beta_{k}, \bar{\beta}_{\boldsymbol{k}^{\prime}}\right]_{+}=\delta_{\boldsymbol{k} \boldsymbol{k}^{\prime}}$ relation can formally be considered as anti-commutation relation for operators giving rise the Fermi space of ghost states. There is no such a possibility for $\bar{\alpha}_{k}, \alpha_{k}$ operators because their anticommutation is negative. If one considers these operators as complete mathematical objects that are not subject to any transformations, then it is impossible to build an operator over them that gives rise to some space of states, and this is because of non-standard anti-commutation relation. The problem is solved by the fact of the existence of Grassman units which are necessary elements of Grassman algebra. At the operator constants level, the bosonization is reduced to the following transformation

$$
\alpha_{k}=u a_{k}, \quad \bar{\alpha}_{k}=\bar{u} a_{k}^{+}, \quad \beta_{k}=\bar{u} b_{k}, \quad \bar{\beta}_{k}=u b_{k}^{+} .
$$

This leads to operators with (96) commutation properties.

The choice of basis is the most significant problem in the interpretation of theory. In the theory of quantum fields of non-stationary Universe, the choice of linear independent basis $f_{k}, f_{k}^{*}$ is ambiguous, in principle. This differentiates it from the theory of quantum fields in the Minkowski space. In the latter, the separation of field into negative and positive frequency components is Lorentz-invariant procedure [31]. A natural physical postulate in accordance to which the definition of particle (quantum of field) in the Minkowski space must be relativistically invariant leads mathematically to

$f_{k}=\left(2 \omega_{k}\right)^{-1 / 2} \mathrm{e}^{-i \omega_{k} t}$. In the non-stationary Universe with the metric (70), the similar postulate can be introduced only for conformally invariant fields and at the level of auxiliary Minkowski space. At the same time, the graviton field is conformally non-invariant. This can be seen from the following. Using the conformal transformation $y_{k}=\tilde{y}_{k} / a$ and transition to the conformal time $d \eta=d t / a$, one can see that Equation (88) is transformed to the equation for the oscillator with variable frequency that reads

$$
\tilde{y}_{k}^{\prime \prime}+\left(k^{2}-\frac{a^{\prime \prime}}{a}\right) \tilde{y}_{k}=0 .
$$

Effects of vacuum polarization and graviton creation in the self-consistent classic gravitational field correspond to parametric excitation of the oscillator (103).

The approximate separation of field on negative and positive frequency components is possible only in the short wavelength limit. Regardless of the background dynamic, linearly independent solutions of Equation (103) exist, and they have the following asymptotes

$$
\tilde{f}_{k} \rightarrow \frac{1}{\sqrt{2 k}} \mathrm{e}^{-i k \eta}, \quad \tilde{f}_{k}^{*} \rightarrow \frac{1}{\sqrt{2 k}} \mathrm{e}^{i k \eta}, \quad k^{2} \gg\left|\frac{a^{\prime \prime}}{a}\right| .
$$

Effects of vacuum polarization and particle creation are negligible for the subsystem of shortwave gravitons. In this sector, quanta of gravitational field can be considered, with a good accuracy, as real gravitons that are situated at their mass shell. The conservation of the number of such real gravitons takes also place with a good accuracy. In the shortwave limit, choosing linear independent solutions of the (104) form, occupation numbers $n_{k \sigma}$ are interpreted as numbers of real gravitons with energy $\varepsilon_{k}=\hbar k / a$, momentum $\boldsymbol{p}=\hbar \mathbf{k} / a$ and polarization $\sigma$. The possibility of such an interpretation is the principle and the only argument in favor of choice of this basis. For the subsystem of shortwave gravitons, initial conditions are permissible not in the form of products of superpositions but in the form of products of state vectors with determined occupation numbers. In accordance with the usual understanding of the status of shortwave ghosts, their state can be chosen in the vacuum form. The gas of shortwave gravitons is described in more detail in Section 4.1.

In the $k^{2} \sim\left|a^{\prime \prime} / a\right|$ vicinity, there is no criterion allowing a choice of preferable basis. It is impossible to introduce the definition of real gravitons in this region because there is no mass shell here. This is the reason why we will use the term "virtual graviton of determined momentum" in discussions of excitations of long wavelengths. Under the term "virtual graviton" we mean a graviton whose momentum is defined but whose energy is undefined. Each set of linear independent solutions corresponds to the distribution of energy for the determined momentum. This distribution can be set up, for example by the expansion of basis function in the Fourier integral. Thus, the choice of basis is, at the same time, the definition of virtual graviton. One needs to mention that different sets of probability amplitudes $\mathcal{C}_{n_{k \sigma}}$ correspond to different definitions of the virtual graviton for the same initial physical state. Note also that limitations that are defined by asymptotes (104) do not fix basis functions completely.

\subsection{One-Loop Finiteness}

The full system of equations of the theory consists of operator equations for gravitons and ghosts (76), (77), macroscopic Einstein equations (78), (79) and Formula (80) for the energy density and pressure of gravitons. The averaging of (80) is carried out over state vectors of general form (94) and (97). The one-loop finiteness is satisfied automatically in this theory. The finiteness is provided by the structure of ghost sector, and it is a result of the following two facts. First, in the space with metric (70) the ghost Equation (77) coincides with graviton Equation (76). Second, the number of internal degrees of freedom of the complex ghost field coincides with that of 3-tensor gravitons. We will show this by direct cal- 
culations.

Let us introduce the graviton spectral function which is renormalized by ghosts. It reads

$$
W_{\boldsymbol{k}}=\sum_{\sigma}\left\langle\Psi_{g}\left|\hat{\psi}_{\boldsymbol{k} \sigma}^{+} \hat{\psi}_{\boldsymbol{k} \sigma}\right| \Psi_{g}\right\rangle-2\left\langle\Psi_{g h}\left|\bar{\theta}_{\boldsymbol{k}} \theta_{\boldsymbol{k}}\right| \Psi_{g h}\right\rangle .
$$

Zero and first moments of this function are the most important objects of the theory. They are

$$
\begin{aligned}
& W_{0}=\sum_{k}\left(\sum_{\sigma}\left\langle\Psi_{g}\left|\hat{\psi}_{\boldsymbol{k} \sigma}^{+} \hat{\psi}_{\boldsymbol{k} \sigma}\right| \Psi_{g}\right\rangle-2\left\langle\Psi_{g h}\left|\bar{\theta}_{\boldsymbol{k}} \theta_{\boldsymbol{k}}\right| \Psi_{g h}\right\rangle\right), \\
& W_{1}=\sum_{k} \frac{k^{2}}{a^{2}}\left(\sum_{\sigma}\left\langle\Psi_{g}\left|\hat{\psi}_{\boldsymbol{k} \sigma}^{+} \hat{\psi}_{\boldsymbol{k} \sigma}\right| \Psi_{g}\right\rangle-2\left\langle\Psi_{g h}\left|\bar{\theta}_{\boldsymbol{k}} \theta_{\boldsymbol{k}}\right| \Psi_{g h}\right\rangle\right) .
\end{aligned}
$$

The energy density and pressure of gravitons that are expressed via moments (106) can be obtained by transformations identical to (80) with use of equations of motion (76) and (77). They read

$$
\begin{aligned}
& \varkappa \varepsilon_{g}=\frac{1}{16} D+\frac{1}{4} W_{1}, \quad \varkappa p_{g}=\frac{1}{16} D+\frac{1}{12} W_{1}, \\
& D=\ddot{W}_{0}+3 H \dot{W}_{0} .
\end{aligned}
$$

In addition, the following relation between moments is derived from equations of motion

$$
\dot{D}+6 H D+4 \dot{W}_{1}+16 H W_{1}=0 .
$$

This relation ensures that the graviton energy-momentum tensor is conservative:

$$
\dot{\varepsilon}_{g}+3 H\left(\varepsilon_{g}+p_{g}\right)=0
$$

As it was shown above, field operators can always be chosen from the basis of complex self-conjugated functions that are the same both for gravitons and ghosts. One needs to also mention that the interpretation of short wave gravitons as real gravitons determines the asymptotic of basis functions (see (104)). After the commutation of operators of creation and annihilation are done, graviton contributions to the moments of the spectral function $W_{n}, n=0,1$ can be presented in the following form

$$
\begin{aligned}
W_{n(\text { grav })} & =\sum_{\boldsymbol{k}} \frac{k^{2 n}}{a^{2 n}} \sum_{\sigma}\left\langle\Psi_{g}\left|\hat{\psi}_{\boldsymbol{k} \sigma}^{+} \hat{\psi}_{\boldsymbol{k} \sigma}\right| \Psi_{g}\right\rangle \\
= & 8 \varkappa \hbar \sum_{\boldsymbol{k}} \frac{k^{2 n}}{a^{2 n}} f_{k}^{*} f_{k} \\
& +4 \varkappa \hbar \sum_{\boldsymbol{k}} \frac{k^{2 n}}{a^{2 n}} \sum_{\sigma}\left(2\left\langle\Psi_{g}\left|\hat{c}_{\boldsymbol{k} \sigma}^{+} \hat{c}_{\boldsymbol{k} \sigma}\right| \Psi_{g}\right\rangle f_{k}^{*} f_{k}\right. \\
& +\left\langle\Psi_{g}\left|\hat{c}_{\boldsymbol{k} \sigma}^{+} \hat{c}_{-\boldsymbol{k}-\sigma}^{+}\right| \Psi_{g}\right\rangle f_{k}^{* 2} \\
& \left.+\left\langle\Psi_{g}\left|\hat{c}_{-\boldsymbol{k}-\sigma} \hat{c}_{\boldsymbol{k} \sigma}\right| \Psi_{g}\right\rangle f_{k}^{2}\right) .
\end{aligned}
$$

In the right-hand-side of (109), the first term is the functional which is independent of the structure of
Heisenberg state vector. It reads

$$
W_{n(\text { grav })}^{(0)}=8 \varkappa \hbar \sum_{k} \frac{k^{2 n}}{a^{2 n}} f_{k}^{*} f_{k}=\frac{4 \varkappa \hbar}{\pi^{2} a^{2 n}} \int_{0}^{\infty} k^{2 n+2} f_{k}^{*} f_{k} \mathrm{~d} k .
$$

The integral (110) describes the contribution of zero oscillations whose spectrum is deformed by macroscopic gravitational field. Asymptotic (104) shows that this integral is diverges. In such a situation, the usual way is to use regularization and renormalization procedures. As a result of these operations, quantum corrections to Einstein equations appear. These corrections are the conformal anomalies and terms that came from Lagrangian $\sim R^{2} \ln \left(R / \lambda_{g}^{2}\right)$ where $\lambda_{g}$ is a scale parameter that comes from renormalization (see Section 10.1). The theory that we present here does not use such operations. There is a contribution of ghost zero oscillations in the moments of spectral function. Its sign is opposite to (110). It reads

$$
\begin{aligned}
& W_{n(g h o s t)}=-2 \sum_{\boldsymbol{k}} \frac{k^{2 n}}{a^{2 n}}\left\langle\Psi_{g h}\left|\bar{\theta}_{\boldsymbol{k}} \theta_{\boldsymbol{k}}\right| \Psi_{g h}\right\rangle \\
& =-8 \varkappa \hbar \sum_{\boldsymbol{k}} \frac{k^{2 n}}{a^{2 n}} f_{k}^{*} f_{k} \\
& -8 \varkappa \hbar \sum_{\boldsymbol{k}} \frac{k^{2 n}}{a^{2 n}}\left(\left\langle\Psi_{g h}\left|\hat{a}_{\boldsymbol{k}}^{+} \hat{a}_{\boldsymbol{k}}+\hat{b}_{\boldsymbol{k}}^{+} \hat{b}_{\boldsymbol{k}}\right| \Psi_{g h}\right\rangle f_{k}^{*} f_{k}\right. \\
& \left.+\left\langle\Psi_{g h}\left|\hat{a}_{\boldsymbol{k}}^{+} \hat{b}_{-\boldsymbol{k}}^{+}\right| \Psi_{g h}\right\rangle f_{k}^{* 2}+\left\langle\Psi_{g h}\left|\hat{b}_{-\boldsymbol{k}} \hat{a}_{\boldsymbol{k}}\right| \Psi_{g h}\right\rangle f_{k}^{2}\right) .
\end{aligned}
$$

The observables (107) are expressed via sums $W_{n(\text { grav })}+W_{n(\text { ghost })}$. In those sums, the exact gravitonghost compensation takes place in the contribution from zero oscillations.

The final expressions for the moments of spectral function are obtained by using the explicit form of state vectors (94) and (97). They read

$$
W_{n}=8 \varkappa \hbar \sum_{\boldsymbol{k}} \frac{k^{2 n}}{a^{2 n}}\left(N_{\boldsymbol{k}}\left|f_{k}\right|^{2}+U_{\boldsymbol{k}}^{*} f_{k}^{* 2}+U_{\boldsymbol{k}} f_{k}^{2}\right),
$$

where

$$
N_{\boldsymbol{k}}=\sum_{\sigma} \sum_{n_{\mathbf{k} \sigma}=1}^{\infty}\left|\mathcal{C}_{n_{\mathbf{k} \sigma}}\right|^{2} n_{\mathbf{k} \sigma}-\sum_{n_{\boldsymbol{k}}=1}^{\infty}\left|\mathcal{A}_{n_{\boldsymbol{k}}}\right|^{2} n_{\boldsymbol{k}}-\sum_{\bar{n}_{\boldsymbol{k}}=1}^{\infty}\left|\mathcal{B}_{\bar{n}_{\boldsymbol{k}}}\right|^{2} \bar{n}_{\boldsymbol{k}}
$$

and

$$
\begin{aligned}
& U_{k}^{*}=\frac{1}{2} \sum_{\sigma}\left(\sum_{n_{k \sigma}=0}^{\infty} \mathcal{C}_{n_{k \sigma}+1}^{*} \mathcal{C}_{n_{k \sigma}} \sqrt{n_{k \sigma}+1}\right) \\
& \times\left(\sum_{n_{k^{\prime} \sigma^{\prime}}=0}^{\infty} \mathcal{C}_{n_{k^{\prime} \sigma^{\prime}+1}}^{*} \mathcal{C}_{n_{k^{\prime} \sigma^{\prime}}} \sqrt{n_{\boldsymbol{k}^{\prime} \sigma^{\prime}}+1}\right) \\
& -\left(\sum_{n_{k}=0}^{\infty} \mathcal{A}_{n_{\mathbf{k}}+1}^{*} \mathcal{A}_{n_{k}} \sqrt{n_{\mathbf{k}}+1}\right) \\
& \times\left(\sum_{\bar{n}_{k^{\prime}}=0}^{\infty} \mathcal{B}_{\bar{n}_{k^{\prime}}+1}^{*} \mathcal{B}_{\bar{n}_{k^{\prime}}} \sqrt{\bar{n}_{\boldsymbol{k}^{\prime}}+1}\right)
\end{aligned}
$$


are spectral parameters. They are defined by initial conditions for the chosen normalized basis of linear independent solutions of Equations (88). (For sake of brevity, in (114) and below we use the following notation $\boldsymbol{k}^{\prime}=-\boldsymbol{k}, \sigma^{\prime}=-\sigma$.) Note that the relation (112) does not contain divergences. Divergences in the relation (112) may appear only because of non-physical initial conditions. The spectrum of real gravitons that slowly decreased for $k \rightarrow \infty$ is an example of such a non-physical initial conditions.

The spectral function (105) depends of three arbitrary constants as it is averaged over the state vector of general form. It reads

$$
W_{\boldsymbol{k}}=8 \varkappa \hbar\left(N_{\boldsymbol{k}}\left|f_{k}\right|^{2}+U_{\boldsymbol{k}}^{*} f_{k}^{* 2}+U_{\boldsymbol{k}} f_{k}^{2}\right) .
$$

In (115), the basis of normalized linear independent solutions contains information on the dynamics of operators of graviton-ghost field; integration constants $N_{k}, U_{k}^{*}, U_{k}$ contain information on the initial ensemble of this field. Due to the background's homogeneity and isotropy the moduli of the amplitudes and average occupation numbers do not depend on the directions of wave vectors and polarizations:

$$
\begin{aligned}
& \left\langle n_{k(g)}\right\rangle=\sum_{n_{\boldsymbol{k} \sigma}=1}^{\infty}\left|\mathcal{C}_{n_{\boldsymbol{k} \sigma}}\right|^{2} n_{\boldsymbol{k} \sigma}, \\
& \left\langle n_{k(g h)}\right\rangle=\sum_{n_{\boldsymbol{k}}=1}^{\infty}\left|\mathcal{A}_{n_{\boldsymbol{k}}}\right|^{2} n_{\boldsymbol{k}}, \\
& \left\langle\bar{n}_{k(g h)}\right\rangle=\sum_{\bar{n}_{\boldsymbol{k}}=1}^{\infty}\left|\mathcal{B}_{\bar{n}_{\boldsymbol{k}}}\right|^{2} \bar{n}_{\boldsymbol{k}} .
\end{aligned}
$$

Phase of amplitudes, in principle, may depend on the directions and polarizations. One must bear in mind that in the pure quantum ensembles, for which the averaging over the state vector is defined, phases of amplitudes are determined. If the phases are random, then the additional averaging should be conducted over them, which corresponds to the density matrix formalism for mixed ensembles. The question of phases of amplitudes is clearly linked to the question of the origin of quantum ensembles. In particular, it is natural to assume that the ensemble of long-wavelength gravitons arises in the process of restructuring graviton vacuum. This process is due to conformal non-invariance of the graviton field and can be described as particle creation. In this case, there is a correlation between the phases of states with the same occupation numbers, but mutually opposite momenta and polarizations: the sum of these phases is zero.

If the typical occupation numbers in the ensemble are large, then squares of moduli of probability amplitudes are likely to be described by Poisson distributions. For this ensemble we get

$$
\begin{aligned}
& \mathcal{C}_{n_{\mathbf{k} \sigma}}=\sqrt{P\left[n_{k(g)}\right]} \exp \left(i \varphi_{n_{\mathbf{k} \sigma}}\right), \\
& \mathcal{A}_{n_{\boldsymbol{k}}}=\sqrt{P\left[n_{k(g h)}\right]} \exp \left(i \chi_{n_{\mathbf{k}}}\right), \\
& \mathcal{B}_{n_{\boldsymbol{k}^{\prime}}}=\sqrt{P\left[\bar{n}_{k(g h)}\right]} \exp \left(i \chi_{\bar{n}_{\mathbf{k}^{\prime}}}\right), \\
& P\left[n_{k}\right]=\frac{\left\langle n_{k}\right\rangle^{n_{k}}}{n_{k} !} \exp \left(-\left\langle n_{k}\right\rangle\right) .
\end{aligned}
$$

The substitution of (116), (117) to (113), (114) leads to

$$
\begin{aligned}
N_{\boldsymbol{k}} & \equiv N_{k}=2\left\langle n_{k(g)}\right\rangle-\left\langle n_{k(g h)}\right\rangle-\left\langle\bar{n}_{k(g h)}\right\rangle . \\
U_{\boldsymbol{k}}^{*} & \equiv U_{k}^{*} \\
& =\left\langle n_{k(g)}\right\rangle \zeta_{k}^{(g)} \mathrm{e}^{i \varphi_{k}}-\sqrt{\left\langle n_{k(g h)}\right\rangle\left\langle\bar{n}_{k(g h)}\right\rangle} \zeta_{k}^{(g h)} \mathrm{e}^{i \chi_{k}}, \\
\zeta_{k}^{(g)} & \leq 1, \zeta_{k}^{(g h)} \leq 1,
\end{aligned}
$$

where

$$
\begin{aligned}
\zeta_{k}^{(g)} \mathrm{e}^{i \varphi_{k}} & =\frac{1}{2} \sum_{\sigma}\left(\sum_{n_{\boldsymbol{k} \sigma}} P\left[n_{k(g)}\right] \exp \left(i \varphi_{n_{\mathbf{k} \sigma}}-i \varphi_{n_{\boldsymbol{k}+1, \sigma}}\right)\right) \\
& \times\left(\sum_{n_{\boldsymbol{k}^{\prime} \sigma^{\prime}}} P\left[n_{k(g)}\right] \exp \left(i \varphi_{n_{\boldsymbol{k}^{\prime} \sigma^{\prime}}}-i \varphi_{n_{\boldsymbol{k}^{\prime}+1, \sigma^{\prime}}}\right)\right), \\
\zeta_{k}^{(g h)} \mathrm{e}^{i \chi_{k}} & =\left(\sum_{n_{\boldsymbol{k}}} P\left[n_{k(g h)}\right] \exp \left(i \chi_{n_{k}}-i \chi_{n_{k+1}}\right)\right) \\
& \times\left(\sum_{\bar{n}_{\boldsymbol{k}^{\prime}}} P\left[\bar{n}_{k(g h)}\right] \exp \left(i \chi_{\bar{n}_{\boldsymbol{k}^{\prime}}}-i \chi_{n_{\boldsymbol{k}^{\prime}+1}}\right)\right)
\end{aligned}
$$

Limit equalities $\zeta_{k}^{(g)}=\zeta_{k}^{(g h)}=1$ are satisfied if the phase difference between states of neighboring occupation numbers does not depend on values of occupation numbers. It is also easy to see that (118) and (119) apply, with somewhat different definitions, to any ensemble with $\zeta_{k}^{(g)} \mathrm{e}^{i \varphi_{k}}$ and $\zeta_{k}^{(g h)} \mathrm{e}^{i \chi_{k}}$ parameters.

We already mentioned above that different basis functions that correspond to different definitions of the virtual graviton can be used for the same initial physical state. Limitations due to the prescriptions on the asymptotic expression (104) allow to fix only asymptotic expansions of basis functions for $k \rightarrow \infty$. These expansions can be used, however, only for description of shortwave modes (Section 4.1). Meanwhile, all non-trivial quantum gravity phenomena take place in spectral region where characteristic wavelengths are of the order of the horizon scale. The choice of basis functions to describe these waves is not unique, and the set of amplitudes of probability $\mathcal{C}_{n_{k \sigma}}$ depends significantly on this set. At the level of Equations (118), (119), the ambiguity in the definition of the virtual graviton reveals itself in the ambiguity of values of parameters $\left\langle n_{k(g)}\right\rangle$ and $\zeta_{k}^{(g)} \mathrm{e}^{i \varphi_{k}}$. Similar ambiguity exists in the ghost sector. Two conclusions follow from that. First, it is necessary to work 
with the state vector of general form, at least during the first stage of the study of the system that contains excitations of long wavelengths. Concretization of the amplitudes $\mathcal{C}_{n_{k \sigma}}$ is possible only after using of additional physical considerations that are different for each concrete case. Second, a theory would be extremely desirable which is invariant with respect to the choice of linear independent solutions of Equation (88), and, correspondingly, is invariant with respect to the choice of amplitudes of probability $\mathcal{C}_{n_{k \sigma}}$ defining the structure of Heisenberg's state vector, respectively. In Section 5, we will show that such a formulation of the theory exists in the form of equation for the spectral function of gravitons renormalized by ghosts. The mathematically equivalent formulation of theory exists in the form of infinite BBGKY chain or hierarchy where joint description of gravitons and ghost is carried out in terms of moments of spectral function $W_{n}, n=0,1,2, \cdots, N \rightarrow \infty$.

\section{Approximate Solutions}

\subsection{Gas of Short Wave Gravitons}

Let us consider the gas of gravitons of wavelength that is much shorter than the distance to the cosmological horizon. We exclude the long waves from the model. Also, the calculation of observables is done approximately, so that non-adiabatic evolution of quantum ensemble is not taken into account. In the framework of these approximations, it is possible to save the pure vacuum status of ghosts because their role is just to provide the one-loop finiteness of macroscopic quantities. Long wave excitations we will consider in Section 4.2.

The calculation of observables for the gas of short wave gravitons can be done by general formulas (107), (112)-(114) after the definition of basis functions and the state vector. For the short wave approximation, the full asymptotic expansion of basis functions exists that satisfies the normalization condition (91) and asymptotes (104). Of course, to use the method of asymptotic expansions, basis functions must be taken in the following form

$$
\begin{aligned}
& f_{k}=\frac{1}{a \sqrt{2 \epsilon_{k}}} \mathrm{e}^{-i \phi_{k}}, \quad f_{k}^{*}=\frac{1}{a \sqrt{2 \epsilon_{k}}} \mathrm{e}^{i \phi_{k}}, \\
& \phi_{k}=\int_{\eta_{0}}^{\eta} \epsilon_{k} \mathrm{~d} \eta,
\end{aligned}
$$

where

$$
\epsilon_{k}=\epsilon_{k}\left(\rho, \rho^{\prime}, \rho^{\prime \prime}, \cdots\right), \quad \rho=-\frac{a^{\prime \prime}}{a}
$$

is a real functional of scale factor and its derivatives. In the short wave approximation, this functional is expanded into the local asymptotic series, which satisfies to the following boundary condition ${ }^{5}$

$$
\epsilon_{k}=\epsilon_{k}\left(\rho, \rho^{\prime}, \rho^{\prime \prime}, \cdots\right) \rightarrow k, \quad \rho, \rho^{\prime}, \rho^{\prime \prime}, \cdots \rightarrow 0 .
$$

There are no arbitrary constants in this expansion if the (122) condition is satisfied.

The following linear ordinary differential equation of the third order with respect to $1 / \epsilon_{k}$ functional follows from the Equation (103) for $y_{k}=a f_{k}, a f_{k}^{*}$ functions

$$
\begin{aligned}
& \frac{1}{2}\left(\frac{1}{\epsilon_{k}}\right)+2 \omega_{k}^{2}\left(\frac{1}{\epsilon_{k}}\right)+\left(\omega_{k}^{2}\right) \frac{1}{\epsilon_{k}}=0, \\
& \omega_{k}^{2}=k^{2}+\rho .
\end{aligned}
$$

The solution of Equation (123) satisfying to the asymptotic condition (122) reads

$$
\frac{1}{\epsilon_{k}}=\frac{1}{\omega_{k}} \sum_{s=0}^{\infty}(-1)^{s} \hat{J}_{k}^{s} \cdot 1 .
$$

Powers of $\hat{J}_{k}$ operator from (124) are defined as follows

$$
\begin{aligned}
& \hat{J}_{k} \cdot \varphi=\frac{1}{4} \int_{\eta_{0}}^{\eta} \frac{\mathrm{d} \eta}{\omega_{k}}\left(\frac{\varphi}{\omega_{k}^{3}}\right), \\
& \hat{J}_{k}^{0} \cdot 1 \equiv 1, \quad J_{k} \cdot 1=\frac{1}{8}\left(-\frac{\rho^{\prime \prime}}{\omega_{k}^{4}}+\frac{5}{4} \frac{\rho^{\prime 2}}{\omega_{k}^{6}}\right), \\
& J_{k}^{2} \cdot 1=J_{k} \cdot\left(J_{k} \cdot 1\right), \quad J_{k}^{s} \cdot 1=J_{k}^{s-1} \cdot\left(J_{k} \cdot 1\right) .
\end{aligned}
$$

The integral is calculated explicitly for arbitrary $s$, so that $J_{k}^{s} \cdot 1$ is a local functional of $\rho$ and its derivatives. It follows from (125) that a small parameter of asymptotic expansion is of the order of $\sim 1 / k^{2}$. The (124) solution is approximate because non-local effects are not included to the local asymptotical series. Calculation of these effects is beyond of limits of this method.

The asymptotic expansion (124), (125) defines the $1 / \epsilon_{k}$ functional, and hence, it defines basis functions (121). The substitution of (121) to (112) produces asymptotic expansions of moments of spectral function that read

$$
\begin{aligned}
& W_{n}=\frac{4 \varkappa \hbar}{a^{2+2 n}} \\
& \cdot \sum_{\boldsymbol{k}} \frac{k^{2 n}}{\epsilon_{k}}\left\{\sum_{\sigma}\left\langle\Psi_{g}\left|c_{\boldsymbol{k} \sigma}^{+} c_{\boldsymbol{k} \sigma}\right| \Psi_{g}\right\rangle-\left\langle\Psi_{g h}\left|a_{\boldsymbol{k}}^{+} a_{\boldsymbol{k}}+b_{\boldsymbol{k}}^{+} b_{\boldsymbol{k}}\right| \Psi_{g h}\right\rangle\right. \\
& +\left[\frac{1}{2} \sum_{\sigma}\left\langle\Psi_{g}\left|c_{\boldsymbol{k} \sigma}^{+} c_{-\boldsymbol{k}-\sigma}^{+}\right| \Psi_{g}\right\rangle-\left\langle\Psi_{g h}\left|a_{\boldsymbol{k}}^{+} b_{-\boldsymbol{k}}^{+}\right| \Psi_{g h}\right\rangle\right] \mathrm{e}^{2 i \phi_{k}} \\
& \left.+\left[\frac{1}{2} \sum_{\sigma}\left\langle\Psi_{g}\left|c_{-\boldsymbol{k}-\sigma} c_{\boldsymbol{k} \sigma}\right| \Psi_{g}\right\rangle-\left\langle\Psi_{g h}\left|b_{-\boldsymbol{k}} a_{\boldsymbol{k}}\right| \Psi_{g h}\right\rangle\right] \mathrm{e}^{-2 i \phi_{k}}\right\} .
\end{aligned}
$$

\footnotetext{
${ }^{5}$ Note that the $\rho^{(n)}(\eta) \rightarrow 0$ asymptotic exists for cosmological solutions of usual interest. For instance, $\rho^{(n)}\left(\eta_{0}\right)=0$ as $\eta_{0}=-\infty$ for the inflation solution. For $\eta_{0}=+\infty$ it takes place for the FRW solution for the Universe filled with ordinary matter.
} 
State vectors from (126) can be concretized from the general considerations. It was mentioned in Section 3.3 that such terms as vacuum, zero oscillations and quantum wave excitations are well defined for the $\rho^{(n)}(\eta) \rightarrow 0$ condition. Under the same condition, state vectors that are built on basis vectors of the Fock space are easily interpreted. First of all, this statement is relevant to gravitons. Eigenvalues $n_{\boldsymbol{k} \sigma}$ and eigenvectors $\left|n_{\boldsymbol{k} \sigma}\right\rangle$ of $\hat{n}_{\mathbf{k} \sigma}=\hat{c}_{\boldsymbol{k} \sigma}^{+} \hat{c}_{\boldsymbol{k} \sigma}$ operator describe real gravitons in asymptotic states. In the short wave approximation, the concept of real gravitons is valid for all other stages of the Universe evolution. Thus, in this particular case, the state vector of the general form can be reduced to the product of vectors corresponding to states with definite graviton numbers $n_{k \sigma}=0,1,2, \cdots$ possessing definite momentum and polarization. It reads

$$
\left|\Psi_{g}\right\rangle=\prod_{k \sigma}\left|n_{k \sigma}\right\rangle .
$$

In asymptotic states, short wave ghosts are only used to compensate non-physical vacuum divergences. In accordance with such an interpretation of the ghost status, we suppose that ghosts and anti-ghosts sit in vacuum states that read

$$
\left|\Psi_{g h}\right\rangle=\prod_{k} \prod_{\boldsymbol{k}^{\prime}}\left|0_{\boldsymbol{k}}\right\rangle\left|\overline{0}_{\boldsymbol{k}^{\prime}}\right\rangle .
$$

Averaging over the quantum state that is defined by (127) and (128) vectors, we get

$$
\begin{aligned}
& \left\langle\Psi_{g}\left|c_{\boldsymbol{k} \sigma}^{+} c_{\boldsymbol{k} \sigma}\right| \Psi_{g}\right\rangle=n_{\mathbf{k} \sigma}, \\
& \left\langle\Psi_{g h}\left|a_{\boldsymbol{k}}^{+} a_{\boldsymbol{k}}\right| \Psi_{g h}\right\rangle=\left\langle\Psi_{g h}\left|b_{\boldsymbol{k}}^{+} b_{\mathbf{k}}\right| \Psi_{g h}\right\rangle=0, \\
& \left\langle\Psi_{g}\left|c_{\boldsymbol{k} \sigma}^{+} c_{-\boldsymbol{k}-\sigma}^{+}\right| \Psi_{g}\right\rangle \\
& =\left\langle\Psi_{g}\left|c_{-\boldsymbol{k}-\sigma} c_{\boldsymbol{k} \sigma}\right| \Psi_{g}\right\rangle=\left\langle\Psi_{g h}\left|a_{\boldsymbol{k}}^{+} b_{-\boldsymbol{k}}^{+}\right| \Psi_{g h}\right\rangle \\
& =\left\langle\Psi_{g h}\left|b_{-\boldsymbol{k}} a_{\boldsymbol{k}}\right| \Psi_{g h}\right\rangle=0, \\
& W_{n}=\frac{4 \varkappa \hbar}{a^{2+2 n}} \sum_{k \sigma} \frac{k^{2 n}}{\epsilon_{k}} n_{\boldsymbol{k} \sigma} .
\end{aligned}
$$

To calculate macroscopic observables in this approximation, it is sufficient to keep only the first terms of expansion of moments of spectral function that contain no higher than second derivative of scale factor. In this approximation, moments of spectral function read

$$
\begin{aligned}
& W_{0}=\frac{4 \varkappa \hbar}{a^{2}} \sum_{\boldsymbol{k} \sigma} \frac{n_{\mathbf{k} \sigma}}{k}, \\
& D=-\frac{8 \varkappa \hbar}{a^{2}}\left(\dot{H}+H^{2}\right) \sum_{\mathbf{k} \sigma} \frac{n_{\mathbf{k} \sigma}}{k}, \\
& W_{1}=\frac{4 \varkappa \hbar}{a^{4}} \sum_{\boldsymbol{k} \sigma} k n_{\mathbf{k} \sigma}+\frac{2 \varkappa \hbar}{a^{2}}\left(\dot{H}+2 H^{2}\right) \sum_{\mathbf{k} \sigma} \frac{n_{\boldsymbol{k} \sigma}}{k},
\end{aligned}
$$

Taking into account (129), we get energy density and pressure of high-frequency graviton gas from (107) that read

$$
\begin{aligned}
& \varkappa \varepsilon_{g}=\frac{2 \hbar}{a^{4}} \sum_{k \sigma} k n_{k \sigma}+\frac{2 \hbar}{2 a^{2}} H^{2} \sum_{k \sigma} \frac{n_{\mathbf{k} \sigma}}{k}, \\
& \varkappa p_{g}=\frac{2 \hbar}{3 a^{4}} \sum_{\boldsymbol{k} \sigma} k n_{\boldsymbol{k} \sigma}-\frac{2 \hbar}{6 a^{2}}\left(2 \dot{H}+H^{2}\right) \sum_{\mathbf{k} \sigma} \frac{n_{\boldsymbol{k} \sigma}}{k} .
\end{aligned}
$$

Relations (129) and (130) are valid if $a^{2} / \bar{k}^{2} \sim \bar{\lambda}^{2} \ll H^{-2}, \quad|\dot{H}|^{-1}$, i.e. the square of ratio of graviton wavelength to horizon distance is much less than unity. In case of large occupation numbers, these results are of the quasi-classical character and can be obtained by the classical theory of gravitational waves [9].

As can be seen from (130), the high-frequency graviton gas differs from the ideal gas with the equation of state $p=\varepsilon / 3$ by only so-called post-hydrodynamic corrections. In accordance with the approximation used, these corrections are of the order of $\bar{\lambda}^{2} H^{2} \ll 1$ in comparison with main terms. Thus, the following simple formula can be used

$$
\varkappa \varepsilon_{g} \simeq 3 \varkappa p_{g} \simeq \frac{C_{g 1}}{a^{4}}, \quad C_{g 1}=\varkappa \hbar \sum_{\mathbf{k} \sigma} k n_{\mathbf{k} \sigma} .
$$

\subsection{Quantized Gravitons and Ghosts of Super-Long Wavelengths}

In the framework of this theory, it is possible to describe the ensemble of super-long gravitational waves $\left(k^{2} \ll\left|a^{\prime \prime} / a\right|\right)$ by an approximate analytical method. Such an ensemble corresponds to the Universe whose observable part is in the chaotic bunch of gravitational waves of wavelengths greater than the horizon distance. The chaotic nature of the bunch is provided by non-zero wave vectors of these waves, so that observable properties of the Universe are formed by superposition of waves of different polarizations and orientations in the space. Such a wave system can produce an the isotropic spectrum and isotropic polarization ensemble consistent with the homogeneity and isotropy of the macroscopic space.

Such an ensemble of super-long waves can be formed only if the size of causally-bounded region is much greater than the horizon distance, which is possible in the framework of the hypothesis of early inflation (or other scenarios (see, e.g. [32]). However, the problem of kinematical stability of an ensemble exists even in the framework of the hypothesis of early inflation. The case is due to the fact that the ensemble of long waves is destroyed during the post-inflation epoch if the Universe is expanded with a deceleration. When long waves come out of horizon, they are transformed to the short waves. Below we show that the kinematical self-stabilization of an ensemble is possible in the framework of self-con- 
sistent theory of long waves.

Long waves under discussion correspond to virtual gravitons. To describe them approximately, one needs to use asymptotic expansions of basis functions over the small parameter $\sim k^{2}$. As well as in the case of short waves, the basis can be chosen in the representation of self-conjugated functions that are parameterized by the universal real functional $\epsilon_{k}(a)$. This preserves the definition (121) and Equation (123). However, due to of our interest in the asymptotical expansion $1 / \epsilon_{k}(a)$ over $\sim k^{2}$ parameter, it is necessary to rewrite Equation (123) in the following form

$$
\left.\left\{a^{2}\left[a^{2}\left(\frac{1}{a^{2} \epsilon_{k}}\right)^{\prime}\right]^{\prime}\right\}^{\prime}\right\}^{\prime}=-4 k^{2} a^{2}\left(\frac{1}{\epsilon_{k}}\right)^{\prime} .
$$

Let us introduce the geometric-dynamic time $d \tau=d \eta / a^{2}$ and the following functional

$$
\frac{1}{2 \epsilon_{k} a^{2}} \equiv \xi_{k}=\sum_{n=0}^{\infty} \xi_{k}^{(n)}
$$

Note that the $\tau$ time coordinate corresponds to the original gauge $\sqrt{-g} g^{00}=1$. Equation (132) and the spectral function (115) now read

$$
\begin{aligned}
& \frac{\mathrm{d}^{3} \xi_{k}}{\mathrm{~d} \tau^{3}}=-4 k^{2} a^{2} \frac{\mathrm{d}}{\mathrm{d} \tau}\left(a^{2} \xi_{k}\right), \\
& W_{\boldsymbol{k}}=8 \varkappa \hbar \xi_{k}\left(N_{\boldsymbol{k}}+U_{\boldsymbol{k}}^{*} \mathrm{e}^{i \Phi_{k}}+U_{\boldsymbol{k}} \mathrm{e}^{-i \Phi_{k}}\right), \\
& \Phi_{k}=\int_{\tau_{k}}^{\tau} \frac{\mathrm{d} \tau}{\xi_{k}},
\end{aligned}
$$

where $\tau_{k}$ is a numerical parameter. Its value is unimportant because the constant's contribution to phases of basis functions is absorbed by phases of contributors that form vectors of the general form (94) and (97). Observables (107) are expressed via moments of spectral function. The latter read

$$
\begin{aligned}
D= & \frac{1}{a^{6}} \sum_{\boldsymbol{k}} \frac{\mathrm{d}^{2} W_{\boldsymbol{k}}}{\mathrm{d} \tau^{2}} \\
= & \frac{8 \varkappa \hbar}{a^{6}} \sum_{\boldsymbol{k}}\left[\frac{\mathrm{d}^{2} \xi_{k}}{\mathrm{~d} \tau^{2}} N_{\boldsymbol{k}}+\left(\frac{\mathrm{d}^{2} \xi_{k}}{\mathrm{~d} \tau^{2}}-\frac{1}{\xi_{k}}\right)\left(U_{\boldsymbol{k}}^{*} \mathrm{e}^{i \Phi_{k}}+U_{\boldsymbol{k}} \mathrm{e}^{-i \Phi_{k}}\right)\right. \\
& \left.\quad+\frac{i}{\xi_{k}} \frac{\mathrm{d} \xi_{k}}{\mathrm{~d} \tau}\left(U_{\boldsymbol{k}}^{*} \mathrm{e}^{i \Phi_{k}}-U_{\boldsymbol{k}} \mathrm{e}^{-i \Phi_{k}}\right)\right], \\
W_{1}= & \frac{1}{a^{2}} \sum_{\boldsymbol{k}} k^{2} W_{\boldsymbol{k}} \\
= & \frac{8 \varkappa \hbar}{a^{2}} \sum_{\boldsymbol{k}} k^{2} \xi_{k}\left(N_{\boldsymbol{k}}+U_{\boldsymbol{k}}^{*} \mathrm{e}^{i \Phi_{k}}+U_{\boldsymbol{k}} \mathrm{e}^{-i \Phi_{k}}\right) .
\end{aligned}
$$

The iteration procedure over $\sim k^{2}$ parameter for Equation (133) is constructed accordingly to the following rules

$$
\begin{aligned}
& \frac{\mathrm{d}^{3} \xi_{k}^{(0)}}{\mathrm{d} \tau^{3}}=0, \quad \xi_{k}^{(0)} \equiv \frac{1}{a^{2} \epsilon_{k}^{(0)}}=P_{k}+R_{k} \tau+Q_{k} \tau^{2}, \\
& \frac{\mathrm{d}^{3} \xi_{k}^{(n)}}{\mathrm{d} \tau^{3}}=-4 k^{2} a^{2} \frac{\mathrm{d}}{\mathrm{d} \tau}\left(a^{2} \xi_{k}^{(n-1)}\right), \quad n \geq 1 .
\end{aligned}
$$

In particular, we get

$$
\frac{\mathrm{d}^{2} \xi_{k}^{(1)}}{\mathrm{d} \tau^{2}}=-2 k^{2} P_{k} a^{4}+\cdots
$$

The virtual graviton is defined by integration constants $P_{k}, Q_{k}, R_{k}$ of the main term of asymptotic expansion. Because the $\epsilon_{k}$ functional of (121) is real (and therefore the $\xi_{k}^{(0)}$ functional of (136) is also real), we obtain following inequality

$$
4 P_{k} Q_{k}-R_{k}^{2}>0, \quad P_{k}>0, \quad Q_{k}>0 .
$$

The dependence of constants $P_{k}, Q_{k}, R_{k}$ and phase $\Phi_{k}$ on $k$ for $k \rightarrow 0$ is defined by the finiteness condition for $k^{2} W_{k}$ and $\mathrm{d}^{2} W_{k} / \mathrm{d} \tau^{2}$, and taking into account the inequality (138) we obtain

$$
\begin{aligned}
P_{k} & =\mathcal{O}\left(k^{-2}\right), & R_{k} & =\mathcal{O}\left(k^{-1}\right), \\
Q_{k} & =\mathcal{O}\left(k^{0}\right), & \Phi_{k} & =\mathcal{O}\left(k^{2}\right) .
\end{aligned}
$$

The main terms of asymptotical expansions of moments (135), energy density and pressure of long wave gravitons can be obtained from (136) for $\xi_{k}^{(0)}$ and (137) for $\xi_{k}^{(1)}$. They read

$$
\begin{aligned}
& D=-\frac{16 C_{g 2}}{a^{2}}+\frac{16 C_{g 3}}{a^{6}}, \quad W_{1}=\frac{8 C_{g 2}}{a^{2}}, \\
& \varkappa \varepsilon_{g}=\frac{C_{g 2}}{a^{2}}+\frac{C_{g 3}}{a^{6}}, \quad \varkappa p_{g}=-\frac{C_{g 2}}{3 a^{2}}+\frac{C_{g 3}}{a^{6}} .
\end{aligned}
$$

where

$$
\begin{aligned}
& C_{g 2}=\varkappa \hbar \sum_{\boldsymbol{k}} k^{2} P_{k}\left(N_{\boldsymbol{k}}+U_{\boldsymbol{k}}^{*}+U_{\boldsymbol{k}}\right), \\
& C_{g 3}=2 \hbar \sum_{\boldsymbol{k}} Q_{k}\left(N_{\boldsymbol{k}}+U_{\boldsymbol{k}}^{*}+U_{\boldsymbol{k}}\right) .
\end{aligned}
$$

For the first time, approximate solutions for the energy density and pressure in the (139) form were obtained for classical long gravitational waves in $[11,12]$. In the theory of classical gravitational waves [11,12], the constants of integration $C_{g 2}$ and $C_{g 3}$ must be positive. The crucial formal difference between classical and quantum long gravitational waves is in the fact that the last ones allow an arbitrary sign of $C_{g 2}$ and $C_{g 3}$ (negative as well as positive). The physics of this crucial difference will be discussed below (Section 4.3).

In a particular case of $\delta$-type graviton spectrum, 
which is localized at the region of very small conformal wave numbers, (139) can be considered as exact solutions. One needs to to go over from summation to integration

$$
\sum_{\boldsymbol{k}} \ldots \rightarrow \frac{1}{(2 \pi)^{3}} \int \mathrm{d}^{3} k \ldots=\frac{1}{2 \pi^{2}} \int_{0}^{\infty} k^{2} \mathrm{~d} k \ldots .
$$

After that, these solutions can be obtained by the following limits

$$
\begin{aligned}
& k^{2} P_{k} \rightarrow \frac{k_{1}}{a_{1}}=\operatorname{const}(k), \quad Q_{k} \rightarrow Q=\operatorname{const}(k), \\
& N_{\boldsymbol{k}}+U_{\boldsymbol{k}}^{*}+U_{\boldsymbol{k}} \rightarrow \frac{2 \pi^{2}}{k^{2}} \mathcal{N}_{0} \delta\left(k-\kappa_{0}\right), \\
& \mathcal{N}_{0}=\operatorname{const}(k), \kappa_{0} \rightarrow 0 .
\end{aligned}
$$

In (141) $k_{1}$ and $a_{1}$ are the constants of dimension of conformal wave number and scale factor, respectively. They provide the correct dimension to parameter $\lim _{k \rightarrow 0} k^{2} P_{k}$.

\subsection{Scenarios of Macroscopic Evolution}

In accordance with (139), the system of long wave gravitons behaves as a medium consisting of two subsystems whose equations of state are $p_{1}=-\varepsilon_{1} / 3$ and $p_{2}=\varepsilon_{2}$. But, the internal structure of this substratum cannot be determined by measurements that are conducted under the horizon of events. The substratum effect (139) on evolution of the Universe, is seen by an observer as an energy density and pressure of the "empty" (non-structured) spacetime, i.e. vacuum. The question is: does the graviton vacuum have a quasi-classic nature, or has its quantum gravity origin been revealed in some cases?

Let us review the situation. First, the superposition of quantum states in state vectors of the general form (94) and (97) could be essentially non-classical. Second, the clearly non-classical ghost sector is inevitably presented in the theory. Its properties are determined by the condition of one-loop finiteness of macroscopic quantities (Section 3.4). The ghost sector is directly relevant to the (139) solution. Let us consider (118) and (119), assuming for the sake of simplicity $\left\langle n_{k(g h)}\right\rangle=\left\langle\bar{n}_{k(g h)}\right\rangle$. Parameters of solution (139) are expressed via parameters of graviton-ghost ensemble as follows

$$
\begin{aligned}
& C_{g 2}=2 \varkappa \hbar \sum_{\boldsymbol{k}} k^{2} P_{k}\left[\left\langle n_{k(g)}\right\rangle\left(1+\zeta_{k}^{(g)} \cos \varphi_{k}\right)\right. \\
& \left.-\left\langle n_{k(g h)}\right\rangle\left(1+\zeta_{k}^{(g h)} \cos \chi_{k}\right)\right] \\
& C_{g 3}=2 \varkappa \hbar \\
& \cdot \sum_{k} Q_{k}\left[\left\langle n_{k(g)}\right\rangle\left(1+\zeta_{k}^{(g)} \cos \varphi_{k}\right)-\left\langle n_{k(g h)}\right\rangle\left(1+\zeta_{k}^{(g h)} \cos \chi_{k}\right)\right]
\end{aligned}
$$

It follows from (142) that $C_{g 2}>0, C_{g 3}>0$ if the graviton contribution dominates over ghosts in the quantum condensate. We will name such a condensate "quasiclassical”. Its energy density is positive, and it can be formed by usual super-long gravitational waves. If the ghost contribution dominates over gravitons in the quantum condensate, then $C_{g 2}<0, C_{g 3}<0$. Such a condensate of negative energy density has no classical analogy.

Summarizing the results of Sections 4.1 and 4.2, we see that in cosmological applications of one-loop quantum gravity we deal with the multi-component system consisting of short wave graviton gas $g 1$ and two subsystems of graviton-ghost condensate $g 2, g 3$. Taking into account (131) and (139), we get the following equation for the scale factor

$$
3 \frac{a^{\prime 2}}{a^{4}}=\frac{C_{g 1}}{a^{4}}+\frac{C_{g 2}}{a^{2}}+\frac{C_{g 3}}{a^{6}} .
$$

In the first scenario, the long wavelength condensate is of negative energy, which means that the contribution of ghost dominates over gravitons. The evolution of such a Universe is of oscillating type. The solution reads

$$
\begin{aligned}
& a^{2}=\frac{C_{g 1}}{2\left|C_{g 2}\right|}+\frac{\sqrt{C_{g 1}^{2}-4 C_{g 2} C_{g 3}}}{2\left|C_{g 2}\right|} \sin \sqrt{\frac{4\left|C_{g 2}\right|}{3} \eta}, \\
& a_{1,2}^{2}=\frac{C_{g 1}}{2\left|C_{g 2}\right|} \mp \frac{\sqrt{C_{g 1}^{2}-4 C_{g 2} C_{g 3}}}{2\left|C_{g 2}\right|}
\end{aligned}
$$

There is no classic analogy to the solution (144). It can be used for scenarios of evolution of the early quantum Universe. In the region of minimal values of the scale factor $a_{\text {min }}=a_{1}$, the $g 3$ condensate bounces the Universe back from a singularity. The transition from the expansion to the contraction epoch at the region of maximal scale factor $a_{\text {max }}=a_{2}$ is provided by $g 2$ condensate. Because of correlation of signs of $C_{g 2}<0$ and $C_{g 3}<0$, the non-singular Universe oscillates. Recent scenarios of oscillating Universes based on condensates of hypothetical ghost fields are under discussion in the current literature as an alternative to the idea of inflation (see, e.g. [32])). Actually, we have shown that the sametype scenario is constructed with the standard building blocks of quantum gravity the well-known FaddeevPopov's ghosts located far from the mass shell. Thus, a very attractive idea is that one and the same mechanism of graviton-ghost condensate formations in the framework of one-loop quantum gravity based on the "standard” Einstein equations (without hypothetical fields and generalizations of Einstein's general relativity) could be responsible for cyclic evolution of the early Universe (instead of inflation).

The second type of scenario applies if gravitons 
dominate over ghosts in the condensate of positive energy. The solution reads

$$
\begin{aligned}
& 2 \sqrt{C_{g 2}\left(C_{g 2} a^{4}+C_{g 1} a^{2}+C_{g 3}\right)}+2 C_{g 2} a^{2}+C_{g 1} \\
& =\left(2 \sqrt{C_{g 2} C_{g 3}}+C_{g 1}\right) \exp \left(\sqrt{\frac{4\left|C_{g 2}\right|}{3} \eta}\right) .
\end{aligned}
$$

The $g 3$ condensate forms the regime of evolution in the vicinity of singularity; meanwhile the asymptote of cosmological solution for $\eta \rightarrow \infty$ is formed by $g 2$ condensate. Short wave gravitons $g 1$ dominate during the intermediate epoch. The ratio of graviton wavelength to horizon distance is constant during the following asymptotical regime

$$
a \sim \exp \left(\sqrt{\frac{\left|C_{g 2}\right|}{3} \eta}\right) \sim t,
$$

This means that the long wave condensate $g 2$ forms the self-consistent regime of evolution that provides its kinematic stability.

\section{BBGKY Hierarchy (Chain) and Exact Solutions of One-Loop Quantum Gravity Equations}

\subsection{Constructing the Chain}

Approximate methods used in Sections 4.1 and 4.2 provide an opportunity to describe only limit cases which are ultra shortwave gravitons and ghosts against the background of almost stable Fock vacuum and superlong wave modes, forming nearly stable graviton-ghost condensate. Now we are examining self-consistent theory of gravitons and ghosts with the wavelengths of the order of distance to the horizon:

$$
\frac{k^{2}}{a^{2}} \sim H^{2},|\dot{H}| .
$$

When describing modes (146), one should keep in mind two factors. First, in the area of the spectrum (146), there are no reasonable approximations, which could be used to solve Equations (76) and (77), if the law of cosmological expansion $a(t), H(t)$ is not known in advance. Second, the (146) modes are quasi-resonant. Quantum gravity processes of vacuum polarization, spontaneous graviton creation by self-consistent field and graviton-ghost condensation are the most intensive in this region of spectrum. From (146) it is also obvious that the threshold for quantum gravitational processes involving zero rest mass gravitons and ghosts is absent. These processes at the scale of horizon occur at any stage of evolution of the Universe, including, in the modern Universe.
The theory that allows quantitatively describe quasiresonant quantum gravitational effects is constructed in the following way. For the spectral function of gravitons and ghosts $W_{k}$, as defined in (105), a differential equation is derived. For this, the first Equation (76) is multiplied by the $\hat{\psi}_{\boldsymbol{k} \sigma}^{+}$(and then by the $\dot{\hat{\psi}}_{\boldsymbol{k} \sigma}^{+}$), conjugated Equation (72) is multiplied by the $\hat{\psi}_{\boldsymbol{k} \sigma}$ (and then by the $\dot{\hat{\psi}}_{\boldsymbol{k} \sigma}$ ); and the equations obtained are averaged and added. Similar action is carried out with equations for ghosts, after which the equations for ghosts are subtracted from the equations for gravitons. These operations yield:

$$
\begin{gathered}
\ddot{W}_{\boldsymbol{k}}-2 F_{\boldsymbol{k}}+3 H \dot{W}_{\boldsymbol{k}}+\frac{2 k^{2}}{a^{2}} W_{\boldsymbol{k}}=0, \\
\dot{F}_{\boldsymbol{k}}=-6 H F_{\boldsymbol{k}}-\frac{k^{2}}{a^{2}} \dot{W}_{\boldsymbol{k}},
\end{gathered}
$$

where

$$
\begin{aligned}
& W_{\boldsymbol{k}}=\sum_{\sigma}\left\langle\Psi_{g}\left|\hat{\psi}_{\boldsymbol{k} \sigma}^{+} \hat{\psi}_{\boldsymbol{k} \sigma}\right| \Psi_{g}\right\rangle-2\left\langle\Psi_{g h}\left|\bar{\theta}_{\boldsymbol{k}} \theta_{\boldsymbol{k}}\right| \Psi_{g h}\right\rangle, \\
& F_{\boldsymbol{k}}=\sum_{\sigma}\left\langle\Psi_{g}\left|\dot{\hat{\psi}}_{\boldsymbol{k} \sigma}^{+} \dot{\hat{\psi}}_{\boldsymbol{k} \sigma}\right| \Psi_{g}\right\rangle-2\left\langle\Psi_{g h}\left|\dot{\bar{\theta}}_{\boldsymbol{k}} \dot{\theta}_{\boldsymbol{k}}\right| \Psi_{g h}\right\rangle .
\end{aligned}
$$

Further, Equation (147) is differentiated. Expressions for $F_{\boldsymbol{k}}, \dot{F}_{\boldsymbol{k}}$ via $W_{\boldsymbol{k}}$ are substituted into the results of differentiation. For the spectral function the third-order equation is produced

$$
\begin{aligned}
& \dddot{W}_{\boldsymbol{k}}+9 H \ddot{W}_{\boldsymbol{k}}+3\left(\dot{H}+6 H^{2}\right) \dot{W}_{\boldsymbol{k}} \\
& +\frac{4 k^{2}}{a^{2}}\left(\dot{W}_{\boldsymbol{k}}+2 H W_{\boldsymbol{k}}\right)=0 .
\end{aligned}
$$

It is now necessary to draw attention to the fact that $W_{k}(t)$ is Fourier image of the two-point function, taken at $t=t^{\prime}$ :

$$
\begin{aligned}
& W\left(t, t^{\prime} ; \boldsymbol{x}-\boldsymbol{x}^{\prime}\right) \\
& =\left\langle\Psi\left|\hat{\psi}_{i}^{k}(t, \boldsymbol{x}) \hat{\psi}_{k}^{i}\left(t^{\prime}, \boldsymbol{x}^{\prime}\right)-2 \bar{\theta}(t, \boldsymbol{x}) \theta\left(t^{\prime}, \boldsymbol{x}^{\prime}\right)\right| \Psi\right\rangle, \\
& W_{\boldsymbol{k}}(t)=\frac{1}{V} \int \mathrm{d}^{3} y W(t, t ; \boldsymbol{y}) \mathrm{e}^{-i \boldsymbol{k} y} .
\end{aligned}
$$

An infinite set of Fourier images is mathematically equivalent to the infinite set of moments of the spectral function

$$
\begin{aligned}
& W_{n} \\
& =\sum_{\boldsymbol{k}} \frac{k^{2 n}}{a^{2 n}}\left(\sum_{\sigma}\left\langle\Psi_{g}\left|\hat{\psi}_{\boldsymbol{k} \sigma}^{+} \hat{\psi}_{\boldsymbol{k} \sigma}\right| \Psi_{g}\right\rangle-2\left\langle\Psi_{g h}\left|\bar{\theta}_{\boldsymbol{k}} \theta_{\boldsymbol{k}}\right| \Psi_{g h}\right\rangle\right), \\
& n=0,1,2, \cdots, \infty .
\end{aligned}
$$

Therefore, from the equation for Fourier images (149), we can move to an infinite system of equations for the moments. For this, Equation (149) is multiplied by $(k / a)^{2 n}$ followed by summation over wave numbers. The result is a Bogoliubov-Born-Green-Kirkwood-Yvon 
(BBGKY) chain. Each equation of this chain connects the neighboring moments:

$$
\begin{aligned}
& \qquad \dot{D}+6 H D+4 \dot{W}_{1}+16 H W_{1}=0, \\
& B(1,2) \\
& \equiv \dddot{W}_{1}+15 H \ddot{W}_{1}+3\left(22 H^{2}+3 \dot{H}\right) \dot{W}_{1} \\
& +2\left(40 H^{3}+18 H \dot{H}+\ddot{H}\right) W_{1}+4 \dot{W}_{2}+24 H W_{2}=0, \\
& B(n, n+1) \\
& \equiv \dddot{W}_{n}+3(2 n+3) H \ddot{W}_{n} \\
& +3\left[\left(4 n^{2}+12 n+6\right) H^{2}+(2 n+1) \dot{H}\right] \dot{W}_{n} \\
& +2 n\left[2\left(2 n^{2}+9 n+9\right) H^{3}+6(n+2) H \dot{H}+\ddot{H}\right] W_{n} \\
& +4 \dot{W}_{n+1}+8(n+2) H W_{n+1}=0, \\
& n=2, \cdots, \infty .
\end{aligned}
$$

Equations (152)-(154) have to be solved jointly with the following macroscopic Einstein equations

$$
\begin{aligned}
& \dot{H}=-\frac{1}{16} D-\frac{1}{6} W_{1}, \\
& 3 H^{2}=\frac{1}{16} D+\frac{1}{4} W_{1}+\varkappa \Lambda .
\end{aligned}
$$

Note that an infinite chain of Equations (152)-(154) contains information not only on the space-time dynamics of field operators, but also about the quantum ensemble, over which the averaging is done. The multitude of solutions of the equations of the chain includes all possible self-consistent solutions of the operator equations, averaged over all possible quantum ensembles. Theory of gravitons presented by BBGKY chain, conceptually and mathematically corresponds to the axiomatic quantum field theory in the Wightman formulation (see Chapter 8 in monograph [25]). Here, as in Wightman, full information on the quantum field is contained in an infinite sequence of averaged correlation functions, definitions of which simply relate to the symmetry properties of manifold, on which this field determines.

In BBGKY chain (152), (153) and (154), unified graviton-ghost objects appear which are moments of the spectral function, renormalized by ghosts. The ghosts are not explicitly labeled so that the chain is can be built formally in the model not containing ghost fields. Mathematical incorrectness of such a model is obvious only with a microscopic point of view because in the quantum theory all the moments of spectral function diverge the stronger, the more the moment number is. The system of Equations (152)-(154) does not "know", however, that without the involvement of ghosts (or something other renormalization procedure) it applies to the mathematically non-existent quantities. The three following mathematical facts are of principal importance.
1) In one-loop quantum gravity, the BBGKY chain can be formally introduced at an axiomatic level;

2) The internal properties of Equations (152)-(155) provide the existence of finite solutions to this system;

3) In finite solutions, there are solutions which do not meet the "classic" condition of positiveness of moments (see Sections 5.2 and 5.3).

It follows from these facts that there should be an opportunity and the need to implement a renormalization procedure to the theory. This procedure should be able to redefine the moments of the spectral function to finite values, but that leaves them sign-undefined. As it can be seen from the theory which is presented in Sections 2 and 3 , in the one-loop quantum gravity such a procedure is contained within the theory under condition that the ghost sector automatically provides the one-loop finiteness.

We found three exact self-consistent solutions of the system of equations consisting of the BBGKY chain (152)(154) and macroscopic given below in Sections 5.2 and 5.3. The existence of exact solutions can be obtained through direct substitution into the original system of equations. The microscopic nature of these solutions, i.e. dynamics of operators and structure of state vector is described in Sections 6 and 7.

\subsection{Graviton-Ghost Condensates of Constant Conformal Wavelength}

In Section 4.2 the exact solution was found for the graviton-ghost condensate, consisting of spatially uniform modes (see (139)-(141)). This solution satisfies to the first two BBGKY Equations (152), (153) for an arbitrary law of evolution $H(t)$ and under condition that $W_{n}=0$ for $n \geq 2$. (Recall that in this solution $D$ and $W_{1}$ must be understood as the result of limit transition $k^{2} \rightarrow 0$; and equality to zero of higher moments follows from the spatial uniformity of modes.) Now we describe the exact self-consistent solutions for the system, in which in addition to spatially uniform modes, quasiresonant modes with a wavelength equal to the distance to the horizon of events are taken into account. In terms of moments of the spectral function, the structure of solutions under discussion is

$$
\begin{aligned}
& D=D(g 2)+D(g 3)+D(g 4), \\
& W_{1}=W_{1}(g 2)+W_{1}(g 4), \quad W_{n}=W_{n}(g 4), n \geq 2, \\
& D(g 3)=\frac{16 C_{g 3}}{a^{6}}, \quad D(g 2)=-\frac{16 C_{g 2}}{a^{2}}, \quad W_{1}(g 2)=\frac{8 C_{g 2}}{a^{2}}, \\
& D(g 4)=-\frac{48 C_{g 4(1)}}{a^{2}} \ln \frac{a_{0}}{\mathrm{e}^{1 / 4} a}, W_{n}(g 4)=\frac{24 C_{g 4(n)}}{a^{2 n}} \ln \frac{a_{0}}{a}, \\
& n=1, \cdots, \infty .
\end{aligned}
$$


Here $C_{g 3}, C_{g 2}, C_{g 4(n)}, a_{0}$ are numerical parameters. Restrictions on their values follow from the condition of the existence of the exact self-consistent solution.

The solution is found by using of the consistency of functions (156) with the relations arising from the macroscopic Einstein's equations (we are discussing model with $\Lambda=0$ ):

$$
\begin{aligned}
& H^{2}=\frac{C_{g 3}}{3 a^{6}}+\frac{C_{g 2}}{3 a^{2}}+\frac{C_{g 4}}{a^{2}} \ln \frac{\mathrm{e}^{1 / 4} a_{0}}{a}, \\
& \dot{H}=-\frac{C_{g 3}}{a^{6}}-\frac{C_{g 2}}{3 a^{2}}-\frac{C_{g 4}}{a^{2}} \ln \frac{\mathrm{e}^{3 / 4} a_{0}}{a}, \\
& \ddot{H}=2 H\left(\frac{3 C_{g 3}}{a^{6}}+\frac{C_{g 2}}{3 a^{2}}+\frac{C_{g 4}}{a^{2}} \ln \frac{\mathrm{e}^{5 / 4} a_{0}}{a}\right) .
\end{aligned}
$$

In (157) as well as further, we use notation $C_{g 4(1)} \equiv C_{g 4}$. Functions $D$ and $W_{1}$ from (156) transform the equation (152) to an identity. The substitution of $W_{1}$ and $W_{2}$ into (153), taking into account (157), leads to the following expression

$$
\begin{aligned}
& B(1,2)=H \frac{48}{a^{4}} \\
& \cdot\left[4\left(C_{g 4(2)}-C_{g 4}^{2}\right) \ln \frac{a_{0}}{a}-\frac{4}{3} C_{g 2} C_{g 4}+C_{g 4}^{2}-2 C_{g 4(2)}\right]=0 .
\end{aligned}
$$

The infinite chain (154), in contrast to the Equation (153), contains moments of spectral functions of quasi-resonant modes. Nevertheless, it does result, only including (158) as a particular case

$$
\begin{aligned}
& B(n, n+1) \\
& =H \frac{48}{a^{2 n+2}}\left[4\left(C_{g 4(n+1)}-C_{g 4} C_{g 4(n)}\right) \ln \frac{a_{0}}{a}\right. \\
& \left.-\frac{4}{3} C_{g 2} C_{g 4(n)}+C_{g 4} C_{g 4(n)}-2 C_{g 4(n+1)}\right]=0, \\
& n=2, \cdots, \infty \text {. }
\end{aligned}
$$

The following relations between parameters follow from (158) and (159)

$$
C_{g 4(n)}=C_{g 4}^{n}, \quad C_{g 2}=-\frac{3}{4} C_{g 4} .
$$

Thus, moments of the spectral function of quasi-resonant modes satisfy to the following recurrent relation

$$
W_{n+1}(g 4)=\frac{C_{g 4}}{a^{2}} W_{n}(g 4)=\left(\frac{C_{g 4}}{a^{2}}\right)^{n} W_{1}(g 4) .
$$

Comparison of (161) with (151) shows that in the exact solution under discussion all quasi-resonant modes

have the same wavelength $\lambda=a / \sqrt{\left|C_{g 4}\right|} \equiv a / k_{0}$. In other words, in the space of conformal wave numbers the spectrum of quasi-resonant wave modes is localized in the vicinity of the fixed value $|k|=k_{0}$.

Depending on the sign of $C_{g 4}$, we get two exact solutions to the macroscopic observables of graviton-ghost media in the form of functionals of scale factor.

1) Oscillating Universe.

Suppose that $C_{g 4}>0$. In accordance with (160), in this case all $C_{g 4(n)}>0$. The positive sign of all moments $W_{n}(g 4)>0$ suggests that gravitons dominate over ghosts in the ensemble of quasi-resonant modes. We also see that the parameter of spatially uniform mode $g 2$ is negative, i.e. $C_{g 2}<0$. As was shown in Section 4.3, signs of parameters of $g 2$ and $g 3$ modes are the same, so $C_{g 3}<0$. From this it follows that ghosts are dominant in case of spatially uniform modes. The energy density and pressure of graviton-ghost substratum read

$$
\begin{aligned}
& \varkappa \varepsilon_{g}=-\frac{\left|C_{g 3}\right|}{a^{6}}+\frac{3 C_{g 4}}{a^{2}} \ln \frac{a_{0}}{a}, \\
& \varkappa p_{g}=-\frac{\left|C_{g 3}\right|}{a^{6}}-\frac{C_{g 4}}{a^{2}} \ln \frac{a_{0}}{\mathrm{e} a} .
\end{aligned}
$$

The parameter $C_{g 2}$ is not explicitly showed up in (162) because it is expressed via $C_{g 4}$ in accordance with (160). There is an oscillating solution to the Einstein equation $3 H^{2}=\varkappa \varepsilon_{g}$ if solutions for the turning points $a_{m}=a_{\min }, a_{\max }$ exist, i.e.

$$
b=\frac{3 C_{g 4} a_{0}^{4}}{4\left|C_{g 3}\right|}>\mathrm{e}, \quad\left(\frac{a_{0}}{a_{m}}\right)^{4}=b \ln \left(\frac{a_{0}}{a_{m}}\right)^{4} .
$$

In the vicinity of turning points energy density is formed by contributions of ghosts and gravitons, which are comparable in their absolute values, but have opposite signs. Far from turning points, graviton quasi-resonant modes dominate. Simplifying the situation, we can say that in the oscillating Universe spatially uniform modes have essentially quantum nature, and quasi-resonant modes allow semi-classical interpretation.

In the absence of a spatially homogeneous subsystem $g 3$, the infinite sequence of oscillations degenerates into one semi-oscillation. Indeed, with $C_{g 3}=0$ the scale factor, as a function of cosmological time, reads

$$
a(\eta)=a_{0} \exp \left(-\frac{C_{g 4} \eta^{2}}{4}\right), \quad C_{g 4}>0 .
$$

In accordance with (164), the Universe originates from a singularity, reaches the state of maximal scale factor $a_{\max }=a_{0}$ and then collapses again to singularity.

2) Birth in Singularity and Accelerating Expansion.

Accordingly to (161), moments of the spectral function of quasi-resonant modes form an alternating sequence if $C_{g 4}<0$. It reads 


$$
W_{n}(g 4)=-(-1)^{n} \frac{24\left|C_{g 4}\right|^{n}}{a^{2 n}} \ln \frac{a}{a_{0}}, \quad n=1, \cdots, \infty .
$$

It is clear that the result (165) can not be obtained for the quasi-classical ensemble of gravitational waves. The microscopic nature of this solution is discussed in Section 6. It is appropriate here to emphasize one more time that the theory, which is formulated in the most common way in the BBGKY form, captures the existence of such a solution.

It is not difficult to notice that the solution which we are now discussing is in a sense, an alternative to the previous solution. With $C_{g 4}<0$, parameters of spatially homogeneous modes are positive $C_{g 2}>0, C_{g 3}>0$. Thus, spatially uniform modes admit semi-classical interpretation, but quasi-resonant modes have essentially quantum nature. The energy density and pressure of graviton-ghost substratum are

$$
\begin{aligned}
& \varkappa \varepsilon_{g}=\frac{C_{g 3}}{a^{6}}+\frac{3\left|C_{g 4}\right|}{a^{2}} \ln \frac{a}{a_{0}}, \\
& \varkappa p_{g}=\frac{C_{g 3}}{a^{6}}-\frac{\left|C_{g 4}\right|}{a^{2}} \ln \frac{\mathrm{e} a}{a_{0}} .
\end{aligned}
$$

Specific properties of solutions to Einstein's equations $3 H^{2}=\varkappa \varepsilon_{g}$ depend on initial conditions and relations between the parameters of graviton-ghost substratum. First of all, let us mention a scenario that corresponds to a singular origin with the strong excitation of spatially uniform modes

$$
C_{g 3} \neq 0, \quad H>0, \quad \frac{3\left|C_{g 4}\right| a_{0}^{4}}{4 C_{g 3}}<\mathrm{e} .
$$

In the case (167), the Universe is born in the singularity and fairly quickly reaches the area of large scale factor values, where it expands with the acceleration:

$$
\begin{aligned}
& a \simeq\left|C_{g 4}\right|^{1 / 2} t \ln ^{1 / 2} \frac{t}{t_{0}}, \quad \frac{\ddot{a}}{a} \simeq \frac{\left|C_{g 4}\right|}{2 a^{2}}, \\
& a \gg a_{0},\left(\frac{C_{3 g}}{3\left|C_{g 4}\right|}\right)^{1 / 4} .
\end{aligned}
$$

Branch of the same solution, with $H<0$ describes the collapsing Universe with a singular end-state.

Two other scenarios correspond to the weak excitation of graviton spatially uniform modes

$$
C_{g 3} \neq 0, \quad \frac{3\left|C_{g 4}\right| a_{0}^{4}}{4 C_{g 3}}>\mathrm{e} .
$$

In the case of (169), the region of legitimate values of the scale factor is divided into two sub-regions

$0 \leq a \leq a_{1}$ and $a_{2} \leq a<\infty$ separated by a barrier of finite width $a_{2}>a_{1}$. In the sub-region of small values of the scale factor, the Universe is born in a singularity, reaches the state with a maximum value of $a=a_{1}$, and then returns to the singularity. In the limit $C_{3 g} \rightarrow 0$ the possibility of such an evolution disappears because of $a_{1} \rightarrow 0$. In sub-region of the large scale factor, the evolution of the Universe starts at the infinite past from the state of zero curvature. At the stage of compression, the Universe reaches the state with a minimum value of $a=a_{2}$, and then turns into an accelerated mode of expansion. With $C_{g 3}=0$, this branch of cosmological solution is described by the following function of cosmological time

$$
a(\eta)=a_{0} \exp \left(\frac{\left|C_{g 4}\right| \eta^{2}}{4}\right), \quad C_{g 4}<0 .
$$

Note that degenerate solutions (164) and (170) differ only in the sign under of exponent.

\subsection{Self-Polarized Graviton-Ghost Condensate in De Sitter Space}

It is easy to find that the system of Equations (152)-(155) has a simple stationary solution $H=$ const , $D=$ const , $W_{n}=$ const . This solution describes the highly symmetrical graviton-ghost substratum that fills the De Sitter space. It reads

$$
\begin{aligned}
& H^{2}=\frac{1}{36} W_{1}+\frac{1}{3} \varkappa \Lambda, \quad a=a_{0} \mathrm{e}^{H t}, \\
& \varepsilon_{g}=-p_{g}=\frac{1}{12} W_{1} .
\end{aligned}
$$

This solution exists both for the $\Lambda=0$ case and for $\Lambda \neq 0$. The first moment of the spectral function satisfies the inequality $W_{1}>-12 \varkappa \Lambda$ is the only independent parameter of the solution. The remaining moments are expressed through by recurrence relations:

$$
\begin{aligned}
& D=-\frac{8}{3} W_{1}, \\
& W_{n+1}=-\frac{n(2 n+3)(n+3)}{2(n+2)} H^{2} W_{n}, \quad n \geq 1 .
\end{aligned}
$$

From (171) and (172) it clearly follows that the solution has essentially vacuum and quantum nature. The first can be seen from the equation of state $p_{g}=-\varepsilon_{g}$. The second can be seen from the fact that the signs of the moments $W_{n+1} / W_{n}<0$ alternate. Another sign of the quantum nature of the effect is contained in the properties of graviton spectrum. The first of recurrence relations allows estimating of wavelengths of gravitons and ghosts that play a dominant part in the formation of observables 


$$
\lambda \sim \frac{a}{\bar{k}} \sim \sqrt{\frac{W_{1}}{\left|W_{2}\right|}}=\frac{1}{H} \sqrt{\frac{3}{10}}=\text { const. }
$$

As can be seen from (173), during the exponential expansion of the Universe typical values of $\bar{k}$ rapidly shift to the region of exponentially large conformal wave numbers. The physical wavelength and macroscopic observables are unchanged in time. Such a situation occurs if the following two conditions apply.

1) In the $\boldsymbol{k}$-space of conformal wave numbers spectra of graviton vacuum fluctuations are flat;

2) In the integration over the flat spectrum, divergent components of integrals excluded for reason to be discussed in Section 6.2. Observables are formed by finite residuals of these integrals.

In Section 6.2, we will show that these conditions are actually satisfied on the exact solution of operator equations of motion, with special choice of Heisenberg's state vector of graviton-ghost vacuum. Microscopic calculation also allows expressing the first moment of spectral function through the curvature of De Sitter space

$$
W_{1}=\frac{9 \varkappa \hbar N_{g}}{2 \pi^{2}} H^{4},
$$

where $N_{g}$ is a functional of parameters of state vector, which is of the order of the number of virtual gravitons and ghosts that are situated under the horizon of events. Their wavelengths are of the order of the distance to the horizon. It must be stressed that the number of gravitons and ghosts $N_{g}$ is a macroscopic value.

The order of magnitude of $N_{g}$ is determined by graviton and ghost numbers in the condensate. Let us emphasize that numbers of gravitons and ghosts and hence, $N_{g}$ parameters are macroscopic qualities. Further down in this section it is assumed that the gravitons dominate in the condensate and that the parameter $N_{g}>0$.

Note that the result (174) can be easily predicted from the general considerations, including considerations of dimension. Indeed, the general formula (112) shows that the moment $W_{1}$ is of dimension $\left[W_{1}\right]=[l]^{-2}$ ([l] is of dimension of length). It also contains the square of the Planck length as a coefficient. Because $W_{1}$ is a functional of the metric, desired dimension can be obtained only using metric's derivatives. It follows from this that $W_{1}=C \cdot \varkappa \hbar H^{4}$ where $C$ dimensionless constant that contains parameters of vacuum condensate. Given (174), the solution in its final form is as follows:

$$
\begin{aligned}
D= & -\frac{12 \varkappa \hbar N_{g}}{\pi^{2}} H^{4}, \\
W_{n}= & \frac{(-1)^{n+1}}{2^{2 n}}(2 n-1) !(2 n+1)(n+2) \\
& \times \frac{2 \varkappa \hbar N_{g}}{\pi^{2}} H^{2 n+2}, \quad n \geq 1 .
\end{aligned}
$$

$$
\varepsilon_{g}=-p_{g}=\frac{3 \hbar N_{g}}{8 \pi^{2}} H^{4},
$$

The macroscopic Einstein's equation is transformed into the equation for the inflation exponent

$$
3 H^{2}=\frac{3 \varkappa \hbar N_{g}}{8 \pi^{2}} H^{4}+\varkappa \Lambda .
$$

Because $N_{g}$ is a macroscopic parameter, the solution under discussion can be directly relevant to the asymptotic future of the Universe. In this case, the number of gravitons and ghosts under the horizon of events and $\Lambda$ term in the Equation (177) should be considered as parameters, whose values were formed during the earlier stages of cosmological evolution. According to Zel'dovich [33], $\Lambda$-term is the total energy density of equilibrium vacuum subsystems of non-gravitational origin. The problem of the $\Lambda$-term formation is so complex that little has changed since the excellent review of Weinberg [34]. We are limited only to showing the order of magnitude of $\Lambda \sim 3 \times 10^{-47} \hbar^{-3} \quad \mathrm{GeV}^{4}$ allowed by observational data.

Some possibilities of co-existence of graviton condensate and $\Lambda$-term will be discussed for $\Lambda \geq 0, N_{g}>0$. (For other possibilities see Section 6.2.) The curvature of the De Sitter space for the asymptotical state of the Universe is calculated by means of the solution to the Equation (177). It reads

$$
\begin{aligned}
& H_{\infty}^{2}=\frac{4 \pi^{2}}{\varkappa \hbar}\left(\frac{1}{N_{g}} \pm \sqrt{\frac{1}{N_{g}^{2}}-\frac{\varkappa^{2} \hbar \Lambda}{6 \pi^{2} N_{g}}}\right), \\
& R=-12 H_{\infty}^{2} .
\end{aligned}
$$

The energy density of vacuum in this state contains contributions of subsystems formed by all physical interactions including the gravitational one

$$
\varepsilon_{\text {vac }}^{(\infty)}=\frac{3 \hbar N_{g}}{8 \pi^{2}} H_{\infty}^{4}+\Lambda .
$$

The relative input of graviton-ghost condensate into asymptotic energy density of the vacuum depends on parameters of the Universe. If the following inequality

$$
\frac{\varkappa^{2} \hbar \Lambda N_{g}}{6 \pi^{2}} \ll 1
$$

applies because of a small number of gravitons and ghosts, then the quantum-gravitational term is small and one must use the following solution

$$
H_{\infty}^{2} \simeq \frac{1}{3} \varkappa \Lambda\left(1+\frac{\varkappa^{2} \hbar \Lambda}{24 \pi^{2}} N_{g}\right) .
$$

If the inequality (180) is satisfied because of a small $\Lambda$-term then the asymptotic state is mostly formed by the graviton-ghost condensate 


$$
H_{\infty}^{2} \simeq \frac{8 \pi^{2}}{\varkappa \hbar N_{g}}-\frac{\varkappa \Lambda}{3} .
$$

It can be seen from (178) for $\Lambda>0$, the number of gravitons and ghosts that can appear in the Universe is limited by maximum value

$$
N_{g(\max )}=\frac{6 \pi^{2}}{\varkappa^{2} \hbar \Lambda} \sim 10^{122} .
$$

In this limiting case (183), the equipartition of the vacuum energy takes place between graviton-ghost and non-gravitational vacuum subsystems

$$
H_{\infty}^{2}=\frac{4 \pi^{2}}{\varkappa \hbar N_{g(\max )}}=\frac{2}{3} \varkappa \Lambda, \quad \varepsilon_{g}^{(\infty)}=\Lambda=\frac{1}{2} \varepsilon_{v a c}^{(\infty)} .
$$

\subsection{The Problem of Quantum-Gravity Phase Transitions}

Three exact solutions of the equations of quantum gravity (with no matter fields and in the absence of $\Lambda$-term) are, in our view, impressive illustrations of physical content of the theory. (Of course, we can not exclude the existence of other exact solutions). The sets of basic formulas (that characterize each of solutions) have the form:

1) Oscillating Universe,

$$
\begin{aligned}
& t=\int_{a_{\min }}^{a} \frac{\mathrm{d} a}{H_{\mathrm{I}}(a)}, \quad H_{I}^{2}\left(a_{\min }\right)=0, \\
& H_{\mathrm{I}}^{2}(a)=\frac{C_{g 3}^{(\mathrm{I})}}{3 a^{6}}+\frac{C_{g 2}^{(\mathrm{I})}}{3 a^{2}}+\frac{C_{g 4}^{(\mathrm{I})}}{a^{2}} \ln \frac{\mathrm{e}^{1 / 4} a_{0}^{(\mathrm{I})}}{a}, \\
& C_{g 4}^{(\mathrm{I})}>0, \quad C_{g 2}^{(\mathrm{I})}=-\frac{3}{4} C_{g 4}^{(\mathrm{I})}<0, \quad C_{g 3}^{(\mathrm{I})} \leq 0, \\
& 3 \frac{\dot{a}^{2}}{a^{2}}=\varkappa \varepsilon_{g}=-\frac{\left|C_{g 3}^{(\mathrm{I})}\right|}{a^{6}}+\frac{3 C_{g 4}^{(\mathrm{I})}}{a^{2}} \ln \frac{a_{0}^{(\mathrm{I})}}{a}, \\
& -6 \frac{\ddot{a}}{a}=\varkappa\left(\varepsilon_{g}+3 p_{g}\right)=-\frac{4\left|C_{g 3}^{(\mathrm{I})}\right|}{a^{6}}+\frac{3 C_{g 4}^{(\mathrm{I})}}{a^{2}} .
\end{aligned}
$$

2) Birth in Singularity and Accelerating Expansion,

$$
\begin{aligned}
& t=\int_{0}^{a} \frac{\mathrm{d} a}{H_{\mathrm{II}}(a)}, \\
& H_{\mathrm{II}}^{2}(a)=\frac{C_{g 3}^{(\mathrm{II})}}{3 a^{6}}+\frac{C_{g 2}^{(\mathrm{II})}}{3 a^{2}}+\frac{C_{g 4}^{(\mathrm{II})}}{a^{2}} \ln \frac{\mathrm{e}^{1 / 4} a_{0}^{(\mathrm{II})}}{a}, \\
& C_{g 4}^{(\mathrm{II})}<0, \quad C_{g 2}^{(\mathrm{II})}=-\frac{3}{4} C_{g 4}^{(\mathrm{II})}>0, \quad C_{g 3}^{(\mathrm{II})} \geq 0, \\
& 3 \frac{\dot{a}^{2}}{a^{2}}=\varkappa \varepsilon_{g}=\frac{C_{g 3}^{(\mathrm{II})}}{a^{6}}+\frac{3\left|C_{g 4}^{(\mathrm{II})}\right|}{a^{2}} \ln \frac{a}{a_{0}^{(I I)}}, \\
& -6 \frac{\ddot{a}}{a}=\varkappa\left(\varepsilon_{g}+3 p_{g}\right)=\frac{4 C_{g 3}^{(\mathrm{II})}}{a^{6}}-\frac{3\left|C_{g 4}^{(\mathrm{II})}\right|}{a^{2}} .
\end{aligned}
$$

3) De Sitter Universe,

$$
\begin{aligned}
& a=a_{0}^{(\mathrm{II})} \mathrm{e}^{H_{\mathrm{II}} t}, \quad H_{\mathrm{II}}^{2}=\frac{8 \pi^{2}}{\varkappa \hbar N_{g}}, \\
& 3 \frac{\dot{a}^{2}}{a^{2}}=\varkappa \varepsilon_{g}=\frac{24 \pi^{2}}{\varkappa \hbar N_{g}}, \\
& -6 \frac{\ddot{a}}{a}=\varkappa\left(\varepsilon_{g}+3 p_{g}\right)=-\frac{48 \pi^{2}}{\varkappa \hbar N_{g}} .
\end{aligned}
$$

If arbitrary shifts in time axis are excluded, then (185) and (186) are 3-parameter solutions $\left(a_{0}, C_{g 4}, C_{g 3}\right)$. Meanwhile (187) does contain one free parameter $N_{g}$. Also one can see that three exact solutions correspond to the spaces of different symmetries. The solution (187) describes 4-space of constant curvature, with the highest possible symmetry. Solution (186) (in the version of appropriate unlimited expansion) describes 4-space, the geometry of which tends asymptotically to the geometry of the Milln space. Finally, the solution (185) (in the version corresponding to oscillations) describes 3-geometry, which is translation-invariant along the axis of time. Different symmetries of different solutions are the rationale for the introduction of phases of graviton-ghost vacuum. It is supposed to be continuous phase transitions between phases with different symmetries.

Representations of phase transitions are, of course, only heuristic nature. In the one-loop quantum gravity, multi-particle correlations in the system of gravitons and ghosts are not taken into account. For this reason, in this theory it is impossible to define the order parameter that plays the role of the master parameter when choosing a phase state. Phase transitions that were discussed above, were actually initiated by disparity between the choice of the asymptotic state and set of the initial conditions. Of course, such operations are meaningful only within the suggestion that the effect of non-equilibrium phase transition will be contained in future theory.

Staying on the heuristic level, we can use the exact solutions (185), (186), (187) to demonstrate in principle the possibility of the existence of equilibrium phase transitions. Let us consider the exact solutions as the various branches of a general solution. A rough phase transition model is the passage from one branch to another while maintaining continuity of scale factor and its first and second derivatives. As can be seen from (185), (186), (187), these conditions provide the equalities of volumes, energies and pressures of gravitonghost systems on both sides of the transition point. It is easy to see that these conditions correspond to the phase transitions of the second kind. The microscopic theory makes it possible to see that at the point of transition the internal structure of graviton-ghost substratum is changed (see Sections 6 and 7). 
Consider consistently simplified models of all of the phase transitions. Graviton-ghost vacuum is of the lowest symmetry in phase (185). This phase is invariant under condition that the shift on the time axis is of the oscillation period only. Phase (186) is of higher symmetry. It is Milln space asymptotically in time at $t \rightarrow \infty$. Phase (187) (graviton-ghost vacuum in the De Sitter space) has the highest symmetry.

Suppose that the symmetry of the graviton-ghost vacuum increases in the process of the universe evolution, that is, the phase transitions occur in the sequence $\mathrm{I} \rightarrow \mathrm{II} \rightarrow \mathrm{III}$. According to (185), (186), the point of transition from the initial state of the oscillating universe I to the state of unlimited expansion II is determined by the following relations

$$
\begin{aligned}
& \varepsilon_{g}^{(\mathrm{I})}\left(a_{c 1}\right)=\varepsilon_{g}^{(\mathrm{II})}\left(a_{c 1}\right) \\
& \rightarrow C_{g 4}^{(\mathrm{I})} \ln \frac{a_{0}^{(\mathrm{I})}}{a_{c 1}}+\left|C_{g 4}^{(\mathrm{II})}\right| \ln \frac{a_{0}^{(\mathrm{II})}}{a_{c 1}}=\frac{1}{3 a_{c 1}^{4}}\left(C_{g 3}^{(\mathrm{II})}+\left|C_{g 3}^{(\mathrm{I})}\right|\right), \\
& \varepsilon_{g}^{(\mathrm{I})}\left(a_{c 1}\right)+3 p_{g}^{(\mathrm{I})}\left(a_{c 1}\right)=\varepsilon_{g}^{(\mathrm{II})}\left(a_{c 1}\right)+3 p_{g}^{(\mathrm{II})}\left(a_{c 1}\right) \\
& \rightarrow C_{g 4}^{(\mathrm{I})}+\left|C_{g 4}^{(\mathrm{II})}\right|=\frac{4}{3 a_{c 1}^{4}}\left(C_{g 3}^{(\mathrm{II})}+\left|C_{g 3}^{(\mathrm{I})}\right|\right),
\end{aligned}
$$

where $a_{c 1}$ is the value of the scale factor at the fitting point, common to the two phases. From (188) one can get the formula for the fitting point and relationship between the parameters of different phases:

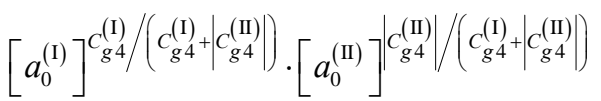

$$
\begin{aligned}
& =\left[\frac{4\left(\left|C_{g 3}^{(\mathrm{I})}\right|+C_{g 3}^{(\mathrm{II})}\right)}{3\left(C_{g 4}^{(\mathrm{I})}+\left|C_{g 4}^{(\mathrm{II})}\right|\right)}\right]^{1 / 4}=a_{c 1} \mathrm{e}^{1 / 4} .
\end{aligned}
$$

As we know, in Phase I gravitons dominate in quasiresonant modes, and ghosts dominate in spatially uniform modes. Following the transition, in Phase II quasi-resonant modes are dominated by ghosts, but spatially uniform modes are dominated by gravitons. Formulas (189) provide constraints on the range of allowed values of the transition point $a_{c 1}$ and the parameters $C_{g 3}^{(\mathrm{II})},\left|C_{g 4}^{(\mathrm{II})}\right|, a_{0}^{(\mathrm{II})}$ of the Phase II for given values of the parameters $\left|C_{g 3}^{(\mathrm{I})}\right|, C_{g 4}^{(\mathrm{I})}, a_{0}^{(\mathrm{I})}$ of Phase I. According to (189), whatever the parameters of Phase I are there is a some set of parameters of a Phase II. Thus, a continuous phase transition I $\rightarrow$ II from the state of oscillating Universe to the state of the Universe in a phase of the unlimited expansion and the asymptotic acceleration is inevitable.

Further, let us consider the phase transition II $\rightarrow$ III . The conditions of sewing together of solutions (186) and (187) read

$$
\begin{aligned}
& \varepsilon_{g}^{(\mathrm{II})}\left(a_{c 2}\right)=\varepsilon_{g}^{(\mathrm{III})} \\
& \rightarrow \frac{C_{g 3}^{(\mathrm{II})}}{a_{c 2}^{6}}+\frac{3\left|C_{g 4}^{(\mathrm{II})}\right|}{a_{c 2}^{2}} \ln \frac{a_{c 2}}{a_{0}^{(\mathrm{II})}}=\frac{24 \pi^{2}}{\varkappa \hbar N_{g}}, \\
& \varepsilon_{g}^{(\mathrm{II})}\left(a_{c 2}\right)+3 p_{g}^{(\mathrm{II})}\left(a_{c 2}\right)=\varepsilon_{g}^{(\mathrm{III})}+3 p_{g}^{(\mathrm{III})} \\
& \rightarrow-\frac{2 C_{g 3}^{(\mathrm{II})}}{a_{c 2}^{6}}+\frac{3\left|C_{g 4}^{(\mathrm{II})}\right|}{2 a_{c 2}^{2}}=\frac{24 \pi^{2}}{\varkappa \hbar N_{g}}
\end{aligned}
$$

In this case, we have the following formulas for the transition point and the relationship between the parameters of the phases:

$$
\begin{aligned}
& \ln \frac{\mathrm{e}^{2}\left[a_{0}^{(\mathrm{II})}\right]^{4}}{a_{c 2}^{4}}=\frac{4 C_{g 3}^{(\mathrm{II})}}{a_{c 2}^{4}\left|C_{g 4}^{(\mathrm{II})}\right|}, \\
& \frac{\left|C_{g 4}^{(\mathrm{II})}\right|}{a_{c 2}^{2}} \ln \frac{\mathrm{e}^{1 / 4} a_{c 2}}{a_{0}^{(\mathrm{II})}}=\frac{12 \pi^{2}}{2 \hbar N_{g}} .
\end{aligned}
$$

According to (191), a continuous phase transition II $\rightarrow$ III is possible if the parameters of Phase II satisfy the inequality

$$
\frac{\mathrm{e}\left[a_{0}^{(\mathrm{II})}\right]^{4}\left|C_{g 4}^{(\mathrm{II})}\right|}{4 C_{g 3}^{(\mathrm{II})}} \geq 1 .
$$

If the phase transition took place, then in Phase III the number of gravitons under the horizon of events is unambiguously defined by parameters of Phase II. The phase transition looks like a "freezing" of the distance to the horizon and of the value of the physical wavelength of quasi-resonant modes.

Finally, we note that a continuous phase transition $\mathrm{I} \rightarrow$ III from the oscillating Universe to De Sitter space is possible if the following conditions are met

$$
\begin{array}{ll}
\ln \frac{\mathrm{e}^{2}\left[a_{0}^{(\mathrm{I})}\right]^{4}}{a_{c 3}^{4}}=\frac{4\left|C_{g 3}^{(\mathrm{I})}\right|}{a_{c 3}^{4} C_{g 4}^{(\mathrm{I})}}, \quad \frac{\mathrm{e}\left[a_{0}^{(\mathrm{I})}\right]^{4} C_{g 4}^{(\mathrm{I})}}{4\left|C_{g 3}^{(\mathrm{I})}\right|} \geq 1, \\
\frac{C_{g 4}^{(\mathrm{I})}}{a_{c 3}^{2}} \ln \frac{\mathrm{e}^{1 / 4} a_{c 3}}{a_{0}^{(\mathrm{I})}}=\frac{12 \pi^{2}}{2 \hbar N_{g}} .
\end{array}
$$

\subsection{Gravitons in the Presence of Matter. Nonlinear Representation of the BBGKY Chain}

The full system of equations of self-consistent theory of gravitons in the isotropic Universe consists of the BBGKY chain (152)-(154) and macroscopic Einstein equations. In Equations (152)-(154), the Hubble function $H$ and its derivatives $\dot{H}, \ddot{H}$ are coefficients multiplied by the moments of the spectral function. In such a form the chain conserves its form even if besides of gravitons, other physical fields are also sources of the macroscopic gravitational field. We are interesting in the 
evolution of the flat isotropic Universe at a stage when the contributions of gravitons and non-relativistic particles, baryons and neutralinos, are quantitatively significant. (The latter are presumably carriers of the mass of Dark Matter.) We assume also that non-gravitational physical interactions created the equilibrium vacuum subsystems with full energy (an effective $\Lambda$-term) of the order of $\Lambda \sim 3 \times 10^{-47} \hbar^{-3} \mathrm{GeV}^{4}$. The macroscopic Einstein equations containing all sources mentioned above read

$$
\begin{aligned}
& R_{0}^{0}-\frac{1}{2} R=\varkappa \varepsilon_{\text {tot }} \\
& \rightarrow H^{2}=\frac{1}{48} D+\frac{1}{12} W_{1}+\frac{\varkappa}{3}\left(\Lambda+\frac{M}{a^{3}}\right), \\
& R_{0}^{0}-\frac{1}{4} R=\frac{3 \varkappa}{4}\left(\varepsilon_{t o t}+p_{t o t}\right) \\
& \rightarrow \dot{H}=-\frac{1}{16} D-\frac{1}{6} W_{1}-\frac{\varkappa M}{2 a^{3}} .
\end{aligned}
$$

Equation (195) should be differentiated with respect to time, and then $\dot{D}$ from (152) should be substituted into the result of differentiation. These operations produce one more equation

$$
\ddot{H}=H\left(\frac{3}{8} D+W_{1}+\frac{3 \varkappa M}{2 a^{3}}\right)+\frac{1}{12} \dot{W}_{1} .
$$

The BBGKY chain (152)-(154) takes into account the interaction of gravitons with the self-consistent classical gravitational field which is represented by the Hubble function and its derivatives. According to Einstein Equations (194)-(196), a self-consistent gravitational field is created by gravitons and other components of cosmological medium, i.e. by the matter and non-gravitational vacuum subsystems. Therefore, the self-consistent gravitational field is a way of describing of significantly non-linear properties of the system that are the result of gravitational interaction of elements of the system. After excluding higher derivatives of the metric from the BBGKY chain (153) and (154), the true non-linear character of the theory emerges. Substitution of (194)(196) into (153) and (154) gives the non-linear representation of BBGKY chain:

$$
\begin{aligned}
& \dot{D}+6 H D+4 \dot{W}_{1}+16 H W_{1}=0, \\
& \dddot{W}_{n}+3(2 n+3) H \ddot{W}_{n}+\left[\frac{1}{16}\left(4 n^{2}+6 n+3\right) D+(n+1)^{2} W_{1}\right. \\
& \left.+\left(8 n^{2}+18 n+9\right) \frac{\varkappa M}{2 a^{3}}+2\left(2 n^{2}+6 n+3\right) \varkappa \Lambda\right] \dot{W}_{n} \\
& +\frac{n}{3}\left\{\frac{1}{2} \dot{W}_{1}+H\left[\frac{n^{2}}{2} D+\left(2 n^{2}+3 n+3\right) W_{1}\right.\right. \\
& \left.\left.+\left(8 n^{2}+18 n+9\right) \frac{\varkappa M}{a^{3}}+4\left(2 n^{2}+9 n+9\right) \varkappa \Lambda\right]\right\} W_{n} \\
& +4 \dot{W}_{n+1}+8(n+2) H W_{n+1}=0, n=1, \cdots, \infty .
\end{aligned}
$$

In the general case, the system of equations (195) and (197) (to which the definition $\dot{a} / a=H$ is added) should be solved numerically with initial conditions determined by the scale factor, moments of the spectral function and their derivatives

$$
\begin{aligned}
& a(0) ; \quad D(0) ; W_{n}(0), \quad \dot{W}_{n}(0), \ddot{W}_{n}(0), \\
& n=1, \cdots, \infty .
\end{aligned}
$$

The initial condition for the Hubble function should be calculated via the equation of the constraint (194)

$$
H(0)=+\sqrt{\frac{1}{48} D(0)+\frac{1}{12} W_{1}(0)+\frac{1}{3} \varkappa \Lambda+\frac{\varkappa M}{3 a^{3}(0)}} .
$$

Any solution of Equations (195) and (197), which corresponds to initial conditions (198), (199), satisfies the identity which is local in time

$$
H^{2}(t)=\frac{1}{48} D(t)+\frac{1}{12} W_{1}(t)+\frac{1}{3} \varkappa \Lambda+\frac{\varkappa M}{3 a^{3}(t)} .
$$

\section{Exact Solutions: Dynamics of Operators and Structure of State Vectors}

In this section, we get the exact solutions for field operators and expressions for the state vectors that correspond to exact analytical solutions of BBGKY chain (185) and (187). Microscopic studies of exact solutions allow greater detail to identify their physical content. Solutions (185) and (187) are formed as a result of certain spectrally dependent correlations between graviton and ghost contributions to the observables. These are full graviton-ghost compensation of contributions of zero oscillations (one-loop finiteness); full compensation of contributions in all parts of the spectrum, except the region of quasi-resonant (QR) and spatially homogeneous (SH) modes; incomplete compensation of contributions of QR and $\mathrm{SH}$ modes with non-zero occupation numbers; correlations between excitation levels and graviton-ghost contents of QR and SH modes, and, finally, some correlations of phases in quantum superpositions of graviton and ghost state vectors.

The physical nature of solution (186) turned out to be unexpected and nontrivial. In Section 7, it will be shown that mathematically this solution describes instanton condensate, which physically corresponds to the system of correlated fluctuations arising during tunneling of graviton-ghost medium between states with fixed difference of graviton and ghost numbers. We explain also that self-polarized graviton-ghost condensate in the De Sitter space also allows instanton interpretation.

\subsection{Condensate of Constant Conformal Wavelength}

Let us consider the solution (185) for $C_{3 g}=0, C_{4 g}=k_{0}^{2}$ : 


$$
H^{2}=\frac{k_{0}^{2}}{a^{2}} \ln \frac{a_{0}}{a}, \quad a=a_{0} \exp \left(-\frac{k_{0}^{2} \eta^{2}}{4}\right) .
$$

The graviton wave equation with the (201) background reads

$$
\hat{\psi}_{\boldsymbol{k} \sigma}^{\prime \prime}-k_{0}^{2} \eta \hat{\psi}_{\boldsymbol{k} \sigma}^{\prime}+k^{2} \hat{\psi}_{\boldsymbol{k} \sigma}=0 .
$$

The equation for the ghosts looks similar. Fundamental solutions of Equation (202) are degenerate hypergeometric functions. It is unnecessary to consider those solutions for all possible values of the parameter $k^{2}$. First of all, it is obvious that the macroscopic observables can be formed only by simplest hypergeometric functions. Values $k^{2}$ that are $k^{2}=0$ (spatially uniform modes) and $k^{2}=k_{0}^{2}$ (quasi-resonant modes) stand out. For all other modes there is a precise graviton-ghost compensation. The reason why it is a mathematically possible follows from the general formulas (115), (118), $(119)^{6}$.

Let us start with quasi-resonant modes. Exact solutions of the Equation (201) and similar equation for ghosts for $k^{2}=k_{0}^{2}$ read

$$
\begin{aligned}
& \hat{\psi}_{\boldsymbol{k} \sigma} \\
& =\frac{\sqrt{4 \varkappa \hbar k_{0}}}{a_{0}}\left[-\eta\left(\hat{Q}_{\boldsymbol{k} \sigma}+k_{0} \hat{P}_{\boldsymbol{k} \sigma} \int_{0}^{\eta} \mathrm{e}^{k_{0}^{2} \eta^{2} / 2} \mathrm{~d} \eta\right)+\frac{\hat{P}_{\boldsymbol{k} \sigma}}{k_{0}} \mathrm{e}^{k_{0}^{2} \eta^{2} / 2}\right] \\
& =-\sqrt{\frac{16 \varkappa \hbar}{k_{0} a_{0}^{2}}}\left[\hat{Q}_{\boldsymbol{k} \sigma}+\hat{P}_{\boldsymbol{k} \sigma} F(a)\right] \ln \ln ^{1 / 2} \frac{a_{0}}{a}, \\
& \hat{\vartheta}_{\boldsymbol{k}}=\frac{\sqrt{4 \varkappa \hbar k_{0}}}{a_{0}}\left[-\eta\left(\hat{q}_{\boldsymbol{k}}+k_{0} \hat{p}_{\boldsymbol{k}} \int_{0}^{\eta} \mathrm{e}^{k_{0}^{2} \eta^{2} / 2} \mathrm{~d} \eta\right)+\frac{\hat{p}_{\boldsymbol{k}}}{k_{0}} \mathrm{e}^{k_{0}^{2} \eta^{2} / 2}\right] \\
& =-\sqrt{\frac{16 \varkappa \hbar}{k_{0} a_{0}^{2}}}\left[\hat{q}_{\boldsymbol{k}}+\hat{p}_{\boldsymbol{k}} F(a)\right] \ln ^{1 / 2} \frac{a_{0}}{a},
\end{aligned}
$$

where $\hat{Q}_{\boldsymbol{k} \sigma}, \hat{P}_{\boldsymbol{k} \sigma}$ and $\hat{q}_{\boldsymbol{k}}, \hat{p}_{\boldsymbol{k}}$ are operators whose properties are defined in (99), (100), (96);

$$
F(a)=a_{0}^{2} \int_{a_{0}}^{a} \frac{\mathrm{d} a}{a^{3} \ln ^{1 / 2} \frac{a_{0}}{a}}-\frac{a_{0}^{2}}{2 a^{2} \ln ^{1 / 2} \frac{a_{0}}{a}} .
$$

Note that one of fundamental solutions to Equation (202) is the Hermite polynomial $H_{1}(\eta)$, which corresponds to positive eigenvalue $k^{2} / k_{0}^{2}=1$. In the re-

\footnotetext{
${ }^{6}$ Formally, all modes except with $k^{2}=0$ and $k^{2}=k_{0}^{2}$, look like "frozen" degrees of freedom, which are excluded from consideration by the model postulate. By virtue of the principle of uncertainty, postulates of this type are outside the formalism of quantum field theory. We want to emphasize that in the finite one-loop quantum gravity there is no need to "freeze" degrees of freedom not participating in the formation of particular exact solutions. Instead of mathematically incorrect operation of "freezing", the formalism of the theory offers mathematically consistent operations of graviton-ghost compensations.
}

production of solutions (186.I) at the microscopic level, this fact is crucial. We will show that the choice of a state vector, satisfying the condition of coherence leads to the fact that only this solution takes part in the formation of the observables. The second solution, containing a function $F(a)$, is a mathematical structure that does not correspond to the exact solution to the BBGKY chain.

Averaging of bilinear forms of operators (203) and (204) over the state vector of the general form leads to the following spectral function

$$
\begin{aligned}
W_{\boldsymbol{k}} & =\sum_{\sigma}\left\langle\Psi_{g}\left|\hat{\psi}_{\boldsymbol{k} \sigma}^{+} \hat{\psi}_{\boldsymbol{k} \sigma}\right| \Psi_{g}\right\rangle-2\left\langle\Psi_{g h}\left|\hat{\vartheta}_{\boldsymbol{k}}^{+} \hat{\vartheta}_{\boldsymbol{k}}\right| \Psi_{g h}\right\rangle \\
& =\frac{16 \varkappa \hbar}{k_{0} a_{0}^{2}}\left[A_{\boldsymbol{k}}+B_{\boldsymbol{k}} F^{2}(a)+C_{\boldsymbol{k}} F(a)\right] \ln \frac{a_{0}}{a} .
\end{aligned}
$$

The constants appearing in (205) are expressed through averaged quadratic forms of operators of generalized coordinates and momentums:

$$
\begin{aligned}
A_{\boldsymbol{k}} & =\sum_{\sigma}\left\langle\Psi_{g}\left|\hat{Q}_{\boldsymbol{k} \sigma}^{+} Q_{\boldsymbol{k} \sigma}\right| \Psi_{g}\right\rangle-2\left\langle\Psi_{g h}\left|\hat{q}_{\boldsymbol{k}}^{+} \hat{q}_{\boldsymbol{k}}\right| \Psi_{g h}\right\rangle, \\
B_{\boldsymbol{k}} & =\sum_{\sigma}\left\langle\Psi_{g}\left|\hat{P}_{\boldsymbol{k} \sigma}^{+} P_{\boldsymbol{k} \sigma}\right| \Psi_{g}\right\rangle-2\left\langle\Psi_{g h}\left|\hat{p}_{\boldsymbol{k}}^{+} \hat{p}_{\boldsymbol{k}}\right| \Psi_{g h}\right\rangle, \\
C_{\boldsymbol{k}} & =\sum_{\sigma}\left\langle\Psi_{g}\left|\left(\hat{Q}_{\boldsymbol{k} \sigma}^{+} P_{\boldsymbol{k} \sigma}+\hat{P}_{\boldsymbol{k} \sigma}^{+} Q_{\boldsymbol{k} \sigma}\right)\right| \Psi_{g}\right\rangle \\
& -2\left\langle\Psi_{g h}\left|\left(\hat{q}_{\boldsymbol{k}}^{+} \hat{p}_{\boldsymbol{k}}+p_{\boldsymbol{k}}^{+} \hat{q}_{\boldsymbol{k}}\right)\right| \Psi_{g h}\right\rangle .
\end{aligned}
$$

Following the transition to the ladder operators in formula (100) and calculations, carried out similar to (112)(120), we get

$$
\begin{aligned}
A_{\boldsymbol{k}} & =2\left\langle n_{k(g)}\right\rangle\left(1+\zeta_{k}^{(g)} \cos \varphi_{k}\right) \\
& -2\left\langle n_{k(g h)}\right\rangle\left(1+\zeta_{k}^{(g h)} \cos \chi_{k}\right), \\
B_{\boldsymbol{k}} & =2\left\langle n_{k(g)}\right\rangle\left(1-\zeta_{k}^{(g)} \cos \varphi_{k}\right) \\
& -2\left\langle n_{k(g h)}\right\rangle\left(1-\zeta_{k}^{(g h)} \cos \chi_{k}\right), \\
C_{\boldsymbol{k}} & =0 .
\end{aligned}
$$

For sake of simplicity, in (207) average numbers of ghosts and anti-ghosts are assumed to be the same: $\left\langle n_{k(g h)}\right\rangle=\left\langle\bar{n}_{k(g h)}\right\rangle$.

Let us go back to the expression (205). Obviously, the spectral function (205) creates moments (161) only if $B_{k}=C_{k}=0$. The condition $C_{k}=0$ is satisfied automatically as a consequence of isotropy of macroscopic state, i.e. because of independence of average occupation numbers of the direction of vector $\boldsymbol{k} . B_{\boldsymbol{k}}=0$ imposes the conditions on amplitudes and phases of quantum superpositions of state vectors with different occupation numbers. It is necessary to draw attention to the fundamental fact: the solution under discussion does not exist, if phases of superpositions are random. Indeed, averaging the expression (207) over phases, we see that condition $B_{\boldsymbol{k}}=0$ is satisfied only if $\left\langle n_{k(g)}\right\rangle=\left\langle n_{k(g h)}\right\rangle$. 
The last equality automatically leads to $A_{\boldsymbol{k}}=0$, i.e. which eliminates the nontrivial solution.

Thus, the condition of the existence of the solution under discussion is the coherence of the quantum state. It is easy to notice (see (120)), that equality $B_{k}=0$, as a condition of coherence, is satisfied for zero phase difference of states with the neighboring occupation numbers of gravitons and ghosts:

$$
\begin{aligned}
& \zeta_{k}^{(g)} \cos \varphi_{k}=\zeta_{k}^{(g h)} \cos \chi_{k}=1 \\
& \rightarrow \zeta_{k}^{(g)}=\zeta_{k}^{(g h)}=1, \quad \cos \varphi_{k}=\cos \chi_{k}=1 .
\end{aligned}
$$

Taking into account (208), we get the following final expression (209) for the spectral function of quasi-resonant gravitons and ghosts

$$
W_{\boldsymbol{k}} \equiv W_{k}=\frac{64 \varkappa \hbar}{k_{0} a_{0}^{2}}\left(\left\langle n_{k(g)}\right\rangle-\left\langle n_{k(g h)}\right\rangle\right) \ln \frac{a_{0}}{a} .
$$

In calculating moments, summation over wave numbers is replaced by integration. Account is taken of that the spectrum as the delta-form with respect to the modulus of $k=|\boldsymbol{k}|$. Also a new parameter $N_{g}$ is introduced where $N_{g}$ is the difference of numbers of gravitons and ghosts in the unit volume of $V=\int \mathrm{d}^{3} x=1$ in the 3-space, which is conformally similar to the 3-space of expanding Universe. Index " $g$ “ in designation of $N_{g}$ parameter indicates the dominance of gravitons in quasi-resonant modes. In accordance with this definition, the following replacement is performed

$$
\left\langle n_{k(g)}\right\rangle-\left\langle n_{k(g h)}\right\rangle \rightarrow \frac{2 \pi^{2}}{k^{2}} N_{g} \delta\left(k-k_{0}\right),
$$

Results of calculating of moments are equated to the relevant expressions of (156) and (161), which were obtained by exact solution of the BBGKY chain:

$$
\begin{aligned}
W_{n}(g 4) & =\frac{1}{2 \pi^{2} a^{2 n}} \int_{0}^{\infty} W_{k} k^{2 n+2} \mathrm{~d} k \\
& =\frac{64 \varkappa \hbar N_{g} k_{0}^{2 n-1}}{a_{0}^{2} a^{2 n}} \ln \frac{a_{0}}{a}=\frac{24 k_{0}^{2 n}}{a^{2 n}} \ln \frac{a_{0}}{a}, \\
D(g 4) & =\frac{1}{a^{2}}\left(W_{0}^{\prime \prime}+2 \frac{a^{\prime}}{a} W_{0}^{\prime}\right) \\
& =-\frac{128 \varkappa \hbar N_{g} k_{0}}{a_{0}^{2} a^{2}} \ln \frac{a_{0}}{\mathrm{e}^{1 / 4} a}=-\frac{48 k_{0}^{2}}{a^{2}} \ln \frac{a_{0}}{\mathrm{e}^{1 / 4} a} .
\end{aligned}
$$

In accordance with (211), there is a relation between parameters $k_{0}, a_{0}$ and $N_{g}$ that appear in the microscopic solution

$$
N_{g}=\frac{3 k_{0} a_{0}^{2}}{8 \varkappa \hbar} .
$$

Recall that in the solution under discussion, the Universe was born in singularity, expands to a state with a maximum scale factor $a_{\max }=a_{0}$, and then is again compressed to the singularity. In this scenario, value $a_{0}$ can be defined as the size of the Universe, accessible for observation in the end stage of expansion. As can be seen from (212), if $a_{0}$ is a macroscopic value, the difference in numbers gravitons and ghosts $N_{g} \gg 1$ is also a macroscopic value.

Contributions of $\mathrm{SH}$ modes to the expressions for the moments are shown in (156), and the relation between the parameters $C_{2 g}$ and $C_{4 g}$ is shown in (160). As a part of the microscopic approach, the construction of exact solutions for these modes is performed by the method of transaction to the limit, described at the end of Section 4.2. The parameter of spatially homogeneous condensate is introduced similarly to (210):

$$
\begin{aligned}
& \left\langle n_{0(g h)}\right\rangle\left(1+\zeta_{0}^{(g h)} \cos \varphi_{0}\right)-\left\langle n_{0(g)}\right\rangle\left(1+\zeta_{0}^{(g)} \cos \chi_{0}\right) \\
& \rightarrow \frac{2 \pi^{2}}{k^{2}} N_{g h} \delta\left(k-\kappa_{0}\right), \quad \kappa_{0} \rightarrow 0 .
\end{aligned}
$$

The index " $g h$ " in $N_{g h}>0$ indicates the dominance of ghosts over the gravitons in the spatially homogeneous condensate. The moments are:

$$
\begin{aligned}
& W_{1}(g 2)=-\frac{16 \varkappa \hbar k_{1} N_{g h}}{a_{1}^{2} a^{2}}, \\
& D(g 2)=\frac{32 \varkappa \hbar k_{1} N_{g h}}{a_{1}^{2} a^{2}} .
\end{aligned}
$$

Definitions of parameters $k_{1}$ and $a_{1}$ are given in (141). The energy density and pressure of the system of QR and SH modes are given by (211) and (214):

$$
\begin{aligned}
\varkappa \varepsilon_{g} & =\frac{8 \varkappa \hbar k_{0} N_{g}}{a_{0}^{2} a^{2}} \ln \frac{a_{0}}{a}+\frac{2 \varkappa \hbar}{a^{2}}\left(\frac{k_{0} N_{g}}{a_{0}^{2}}-\frac{k_{1} N_{g h}}{a_{1}^{2}}\right) \\
& =\frac{8 \varkappa \hbar k_{0} N_{g}}{a_{0}^{2} a^{2}} \ln \frac{a_{0}}{a}, \\
\varkappa p_{g} & =-\frac{8 \varkappa \hbar k_{0} N_{g}}{3 a_{0}^{2} a^{2}} \ln \frac{a_{0}}{\mathrm{e} a}-\frac{2 \varkappa \hbar}{3 a^{2}}\left(\frac{k_{0} N_{g}}{a_{0}^{2}}-\frac{k_{1} N_{g h}}{a_{1}^{2}}\right) \\
& =-\frac{8 \varkappa \hbar k_{0} N_{g}}{3 a_{0}^{2} a^{2}} \ln \frac{a_{0}}{\mathrm{e} a} .
\end{aligned}
$$

In Formula (215), the terms in brackets are eliminated by the condition (160), which is rewritten in terms of microscopic parameters

$$
\frac{k_{0} N_{g}}{a_{0}^{2}}=\frac{k_{1} N_{g h}}{a_{1}^{2}} .
$$

The solution (215), (216) describes a quantum coherent condensate of quasi-resonant modes with graviton dominance, parameters of which are consistent with that of spatially homogeneous condensate with the ghost dominance. 


\subsection{Condensate of Constant Physical Wavelength}

The De Sitter solution for plane isotropic Universe reads

$$
a=a_{0} \mathrm{e}^{H t}=-\frac{1}{H \eta}, \quad H=\text { const. }
$$

For the background (217), the gravitons and ghost equations and their solutions read

$$
\begin{aligned}
& \hat{\psi}_{\boldsymbol{k} \sigma}^{\prime \prime}-\frac{1}{\eta} \hat{\psi}_{\boldsymbol{k} \sigma}^{\prime}+k^{2} \hat{\psi}_{\boldsymbol{k} \sigma}=0, \\
& \hat{\psi}_{\boldsymbol{k} \sigma}=\frac{1}{a} \sqrt{\frac{2 \varkappa \hbar}{k}}\left[c_{\boldsymbol{k} \sigma} f(x)+c_{-\boldsymbol{k}-\sigma}^{+} f^{*}(x)\right], \\
& \hat{\vartheta}_{\boldsymbol{k}}^{\prime \prime}-\frac{1}{\eta} \hat{\vartheta}_{\boldsymbol{k}}^{\prime}+k^{2} \hat{\vartheta}_{\boldsymbol{k}}=0, \\
& \hat{\vartheta}_{\boldsymbol{k}}=\frac{1}{a} \sqrt{\frac{2 \varkappa \hbar}{k}}\left[a_{\boldsymbol{k}} f(x)+b_{-\boldsymbol{k}}^{+} f^{*}(x)\right],
\end{aligned}
$$

where

$$
f(x)=\left(1-\frac{i}{x}\right) \mathrm{e}^{-i x}, \quad x=k \eta .
$$

Ladder operators in (218), (219), have the standard property of (93), (96), which allow their use of in constructing build basic vectors for the Fock space from which the general state vectors are constructed.

The self-consistent dynamics of gravitons and ghosts in the De Sitter space are not trivial in the sense that the averaged bilinear forms of operators (218), (219) which are explicitly and essentially depending on time, must lead to time-independent macroscopic observables. It must be emphasized, that the existence of such, at first glance unlikely solution, is guaranteed by the existence of the solution for the BBGKY chain. The key to the solution lies in the structure of the state vectors of gravitons and ghosts.

Substitution of operator functions (218), (219) into the general expression for the moments (151) yields:

$$
\begin{aligned}
& W_{n}=\frac{2 \varkappa \hbar}{\pi^{2}} H^{2 n+2} \\
& \cdot \int_{0}^{\infty} \mathrm{d} x x^{2 n+1}\left\{U_{\boldsymbol{k}(\text { wave })}|f(x)|^{2} \mathbb{Y}+U_{\boldsymbol{k}(\text { cr })}\left[f^{*}(x)\right]^{2},\right. \\
& \left.\quad+U_{\boldsymbol{k}(\text { ann })}[f(x)]^{2}\right\}
\end{aligned}
$$

where

$$
\begin{aligned}
& N_{\boldsymbol{k}} \equiv U_{\boldsymbol{k}(\text { wave })} \\
& =\sum_{\sigma}\left\langle\Psi_{g}\left|c_{\boldsymbol{k} \sigma}^{+} c_{\boldsymbol{k} \sigma}\right| \Psi_{g}\right\rangle-\left\langle\Psi_{g h}\left|a_{\boldsymbol{k}}^{+} a_{\boldsymbol{k}}\right| \Psi_{g h}\right\rangle \\
& -\left\langle\Psi_{g h}\left|b_{\boldsymbol{k}}^{+} b_{\boldsymbol{k}}\right| \Psi_{g h}\right\rangle ;
\end{aligned}
$$

$$
\begin{aligned}
& U_{\boldsymbol{k}}^{*} \equiv U_{\boldsymbol{k}(c r)} \\
& =\frac{1}{2} \sum_{\sigma}\left\langle\Psi_{g}\left|c_{\boldsymbol{k} \sigma}^{+} c_{-\boldsymbol{k}-\sigma}^{+}\right| \Psi_{g}\right\rangle-\left\langle\Psi_{g h}\left|a_{\boldsymbol{k}}^{+} b_{-\boldsymbol{k}}^{+}\right| \Psi_{g h}\right\rangle ; \\
& U_{\boldsymbol{k}} \equiv U_{\boldsymbol{k}(a n n)} \\
& =\frac{1}{2} \sum_{\sigma}\left\langle\Psi_{g}\left|c_{-\boldsymbol{k}-\sigma} c_{\boldsymbol{k} \sigma}\right| \Psi_{g}\right\rangle-\left\langle\Psi_{g h}\left|b_{-\boldsymbol{k}} a_{\boldsymbol{k}}\right| \Psi_{g h}\right\rangle \\
& \equiv U_{\boldsymbol{k}(c r) .}^{*} .
\end{aligned}
$$

Here $U_{\boldsymbol{k} \text { (wave) }}$ is the spectral parameter of quantum waves, which become real gravitons if $k \eta \gg 1 ; U_{\boldsymbol{k}(c r)}$, $U_{\boldsymbol{k}(a n n)}$ are the spectral parameters of quantum fluctuations that emerge in the processes of graviton (and ghost) creation from the vacuum and graviton (and ghost) annihilation to the vacuum.

Obviously, at the first stage of calculations we assume that the averaging in (221), (222) is conducted over the state vectors of the general form (94), (97). This allows us to go to formulas (113), (114) or (118)-(120). Then it is necessary to take into account that the moments $W_{n}$ must not depend on time, and that they also should be free of divergences. When analyzing the conditions for these demands, the specific form of the expression (220) plays an important part. The measure of integration and the dependence of field operators on the wave number and time can be represented in the terms of the variable $x=k \eta$. A separate (additional) dependence on the wave number can be connected with the structure of spectral parameters. After substitution of the variable $k=x / \eta$ in the equation (221), it is seen that the first term in (220) is time-independent only if $U_{\boldsymbol{k} \text { (wave) }}$ is independent on the wave number. This means that the graviton and ghost spectra must be flat. However, with the flat spectrum there is danger of divergences: if

$U_{\boldsymbol{k} \text { (wave) }}=$ const $(\boldsymbol{k}) \neq 0$, then the first integral in (220) does not exist, because $|f(x)|^{2} \rightarrow 1$ with $x \rightarrow \infty$.

The divergences can be avoided only with exact compensation of contributions from gravitons and ghosts to the spectral parameter $U_{\boldsymbol{k} \text { (wave) }}$. Let us point out, that in that case we are not talking about zero oscillations but about the contributions from the states with non-zero occupation numbers. The compensation condition leading to $U_{\boldsymbol{k} \text { (wave) }}=0$ is:

$$
\left|\mathcal{C}_{n_{\mathbf{k} \sigma}}\right|=\left|\mathcal{A}_{n_{\mathbf{k}}}\right|=\left|\mathcal{B}_{n_{-\boldsymbol{k}}}\right| \text {. }
$$

The result (223) has a simple physical interpretation. The quantum waves of gravitons and ghosts with the equation of state which differs from $p=-\varepsilon$ can not be carriers of energy in the De Sitter space with the self-consistent geometry. The total energy of quantized waves is equal to zero due to exactly the same number of gravitons and ghosts in all regions of the spectrum: 


$$
\left\langle n_{k \sigma_{1}}\right\rangle+\left\langle n_{k \sigma_{2}}\right\rangle=\left\langle n_{\boldsymbol{k}}\right\rangle+\left\langle\bar{n}_{\boldsymbol{k}}\right\rangle .
$$

With equal polarizations of gravitons and the equality of numbers of ghosts and anti-ghosts, it follows from (224) that $\left\langle n_{\boldsymbol{k}(g)}\right\rangle=\left\langle n_{\boldsymbol{k}(g h)}\right\rangle$. Exact equality of the average number of gravitons and ghost is a characteristic feature of the De Sitter space with the self-consistent geometry. Let us mention that for the solution discussed in the previous Section 6.1, that equality is absent in principle. It means that different solutions have different microscopic structures of the graviton-ghost condensate.

Based on the reasoning analogous to the one described above, spectrum parameters $U_{\boldsymbol{k}(c r)}, U_{\boldsymbol{k}(a n n)}$ also must not depend on the wave vector $\boldsymbol{k}$. However, the corresponding integrals in the second and third terms of (220) are not divergent. The absence of divergences is due to the fact that with $x \rightarrow \infty$ the integration is taken over the fast oscillating functions $\sim \mathrm{e}^{ \pm 2 i x}$. To calculate these integrals, they should be additionally defined as follows:

$$
\begin{aligned}
& \lim _{\zeta \rightarrow 0} \int_{0}^{\infty} \mathrm{d} x x^{2 n \pm 1} \mathrm{e}^{-(\zeta-2 i) x}=\mp(-1)^{n} \frac{(2 n \pm 1) !}{2^{2 n+1 \pm 1}}, \\
& 2 i \lim _{\zeta \rightarrow 0} \int_{0}^{\infty} \mathrm{d} x x^{2 n} \mathrm{e}^{-(\zeta-2 i) x}=(-1)^{n+1} \frac{(2 n) !}{2^{2 n}} .
\end{aligned}
$$

At every instant of time, the procedure of redefinitions of integrals (225) selects the contributions from virtual gravitons and ghosts with a characteristic wavelength (173) and eliminate the contributions of all other graviton-ghost modes. This redefining procedure provides the existence of recursive relations (172) in the exact solution of the BBGKY chain.

Thus, in (220) we have a flat spectrum of gravitons and ghosts, $U_{\boldsymbol{k}(\text { wave })} \equiv 0, U_{\boldsymbol{k}(c r)}=U_{\boldsymbol{k}(\text { ann })}^{*}=U=\operatorname{const}(k)$. The expression for the spectral parameter takes the form:

$$
\begin{aligned}
& U=\left(\sum_{n} \mathcal{C}_{n+1}^{*} \mathcal{C}_{n} \sqrt{n+1}\right)^{2} \\
& -\left(\sum_{n} \mathcal{A}_{n+1}^{*} \mathcal{A}_{n} \sqrt{n+1}\right)\left(\sum_{n} \mathcal{B}_{n+1}^{*} \mathcal{B}_{n} \sqrt{n+1}\right), \\
& \left|\mathcal{C}_{n}\right|=\left|\mathcal{A}_{n}\right|=\left|\mathcal{B}_{n}\right| \equiv \sqrt{\mathcal{P}_{n}},
\end{aligned}
$$

where $\mathcal{P}_{n}$ is a normalized statistical distribution. The average value of the number of gravitons and ghosts, having the wavelength in the vicinity of characteristic values (173), are calculated by the formula

$$
\left\langle n_{g}\right\rangle=\left\langle n_{g h}\right\rangle=\langle n\rangle=\sum_{n=0}^{\infty} n \mathcal{P}(n) .
$$

Using the Poisson distribution in (226), (227), the values of integrals (225) and the formulas (119), (120), we get the moments

$$
\begin{aligned}
D & =-\frac{12 \varkappa \hbar N_{g}}{\pi^{2}} H^{4}, \\
W_{n}= & \frac{(-1)^{n+1}}{2^{2 n}}(2 n-1) !(2 n+1)(n+2) \\
& \times \frac{2 \varkappa \hbar N_{g}}{\pi^{2}} H^{2 n+2}, \\
n & \geq 1,
\end{aligned}
$$

where

$$
\begin{aligned}
& N_{g}=\langle n\rangle\left(\zeta_{g} \cos \varphi-\zeta_{g h} \cos \chi\right), \\
& \varphi=\varphi_{n_{k \sigma}}-\varphi_{n_{k+1, \sigma}}, \quad \chi=\chi_{n_{k}}-\chi_{n_{k+1}} .
\end{aligned}
$$

Zero moment $W_{0}$, which has an infrared logarithmic singularity, is not contained in the expressions for the macroscopic observables, and for that reason, is not calculated. In the equation for $W_{0}$, the functions are differentiated in the integrand and the derivatives are combined in accordance with the definition $D=\ddot{W}_{0}+3 H \dot{W}_{0}$. At the last step the integrals that are calculated, already posses no singularities.

Averaging of the parameter (229) over the phases yields $N_{g}=0$. Therefore the solution under discussion does not exist if the superposition of the phases are random. The coherence of the quantum ensemble, i.e. the correlation of phases in the quantum superposition of the basic vectors, corresponding to the different occupation numbers, points to the fact that the medium is in the graviton-ghost condensate state. The gravitons are dominant in the condensate if $N_{g}>0$, and the ghosts are dominant if $N_{g}<0$.

The duality of the condensate and the indeterminate sign of the $\Lambda$-term create different evolutional scenarios. Of course, all these scenarios are present in the expression (178), which is obtained as a solution of the macroscopic Einstein equation (177). In addition to the scenarios described in the Section 5.3, we will show the possibility of strong renormalization of energy of nongravitational vacuum subsystems by the energy of the graviton-ghost condensate ${ }^{7}$.

We have in mind a situation, in which the modulus of $\Lambda$-term exceeds the density of vacuum energy in the asymptotic state of the Universe by many orders of magnitude:

$$
\frac{|\Lambda|}{\varepsilon_{\text {vac }}^{(\infty)}} \equiv \frac{\varkappa|\Lambda|}{3 H_{\infty}^{2}}=\mathcal{N} \gg 1,
$$

where $\mathcal{N}$ is a huge macroscopic number. From (178) it follows that the effect of strong renormalization takes place if

\footnotetext{
${ }^{7}$ Mechanisms that are able to drive the cosmological constant to zero have been discussed for decades (see $[34,38]$ for a review). Any particular scenarios were considered in [37,39-42].
} 


$$
\begin{aligned}
& \frac{\Lambda}{N_{g}}<0, \quad\left|N_{g}\right| \gg \frac{6 \pi^{2}}{\varkappa^{2} \hbar|\Lambda|}, \\
& \varepsilon_{v a c}^{(\infty)} \simeq 2 \pi \sqrt{\frac{6|\Lambda|}{\varkappa^{2} \hbar\left|N_{g}\right|}} .
\end{aligned}
$$

Let us mention that the strong renormalization of the positive $\Lambda$-term is provided by a condensate in which the ghosts are dominant, and for the negative $\Lambda$-termby a condensate for which the gravitons are dominant.

For clarity and for the evaluations let us introduce the Plank scale $M_{P l}=(8 \pi \hbar / \varkappa)^{1 / 2}=1.22 \times 10^{19} \mathrm{GeV}$, the scale of $\Lambda$-term $M_{\Lambda}=\left(\hbar^{3}|\Lambda|\right)^{1 / 4}$, and the scale of the density of Dark Energy in the asymptotical state of the Universe, $M_{D E}=\left(\hbar^{3} \varepsilon_{v a c}^{(\infty)}\right)^{1 / 4}$. We discuss the case when $M_{D E} \ll M_{\Lambda}$.

If non-gravitational contributions to $\Lambda$-term are selfompensating, then a realistic estimate of the $M_{\Lambda}$-scale can be based on the Zeldovich remark [33]. According to [33], non-gravitational $\Lambda$-term is formed by gravitational exchange interaction of quantum fluctuations on the energy scale of hadrons. In terms of contemporary understanding of hadron's vacuum, the focus should be on non-perturbative fluctuations of quark and gluon fields, forming a quark-gluon condensate (see [35,36]). In this case, $\Lambda$-term is expressed only through the minimum and maximum scales of particle physics which are the QCD scale $M_{O C D} \simeq 215 \mathrm{MeV}$ and Planck scale $M_{P l}=1.22 \times 10^{19} \mathrm{GeV}$ :

$$
\hbar^{3}|\Lambda|=M_{\Lambda}^{4}=\frac{M_{Q C D}^{6}}{M_{P l}^{2}} \simeq 10^{-42} \mathrm{GeV}^{4} .
$$

In terms of these scales, it is turns out that a large number of $\mathcal{N}=M_{\Lambda}^{4} / M_{D E}^{4} \sim 10^{5}$, which is defined in (230), can be obtained by the huge number of $\left|N_{g}\right|^{1 / 2}$, for the same number of orders of magnitude greater than the ratio $\left(M_{P l} / M_{\Lambda}\right)^{2}$. Indeed, choosing $\mathcal{N}$, we find

the value of $\left|N_{g}\right|$, which determines the ratio of vacuum energy density to the true cosmological constant in the asymptotic state:

$$
\mathcal{N}=\frac{M_{\Lambda}^{2}}{M_{P l}^{2}} \sqrt{\frac{2\left|N_{g}\right|}{3}} .
$$

The vacuum energy density of asymptotical state is calculated as follows

$$
\varepsilon_{v a c}^{(\infty)} \simeq \hbar^{-3} M_{P l}^{2} M_{\Lambda}^{2} \sqrt{\frac{3}{2\left|N_{g}\right|}} .
$$

Thus, the macroscopic effect of quantum gravity-the condensation of gravitons and ghosts into the state with a certain wavelength of the order of the horizon scaleplays a significant role in the formation of the asymptotic values of energy density of cosmological vacuum. The current theory explains how the strong renormalization of the vacuum energy occurs, but, unfortunately, it does not explain why this happens and why the quantitative characteristics of the phenomenon are those that are observed in the modern Universe. Of the general considerations one can suggest that the coherent gravitonghost condensate occurs in the quantum-gravitational phase transition (see Section 5.4), and the answers to questions should be sought in the light of the circumstances.

\section{Gravitons and Ghosts as Instantons}

\subsection{Self-Consistent Theory of Gravitons in Imaginary Time}

\subsubsection{Invariance of Equations of the Theory with Respect to Wick Rotation of Time Axis}

As has been repeatedly pointed out, the complete system of equations of the theory consists of the BBGKY chain (152)-(154) and macroscopic Einstein's Equation (155). On the basis of common mathematical considerations, it can be expected that solutions to these equations covers every possible self-consistent states of quantum subsystem of gravitons and ghosts and the classical subsystem of macroscopic geometry as well. In examining the model that operates with the pure gravity (no matter fields and $\Lambda$-term), one can identify the following unique property of the theory. Equations of the theory (152)(155) are invariant with respect to the Wick time axis rotation, conducted jointly with the multiplicative transformation of moments of the spectral function:

$$
\begin{array}{ll}
t \rightarrow i \tau, & H \rightarrow-i \mathcal{H}, \\
D \rightarrow-\mathcal{D}, & W_{n} \rightarrow(-1)^{n} \mathcal{W}_{n} .
\end{array}
$$

Rules of transformation of time derivatives are obtained from (235)

$$
\begin{aligned}
& \dot{H} \rightarrow-\dot{\mathcal{H}}, \quad \ddot{H} \rightarrow i \ddot{\mathcal{H}}, \quad \dot{D} \rightarrow i \dot{\mathcal{D}}, \\
& \dot{W}_{n} \rightarrow-i(-1)^{n} \dot{\mathcal{W}}_{n}, \quad \ddot{W}_{n} \rightarrow-(-1)^{n} \ddot{\mathcal{W}}_{n}, \\
& \dddot{W}_{n} \rightarrow i(-1)^{n} \dddot{\mathcal{W}}_{n} .
\end{aligned}
$$

In (236) and further on we use the notation $\dot{\mathcal{F}}=\mathrm{d} \mathcal{F} / \mathrm{d} \tau$. The statement about the invariance of the theory can proved by direct calculations. As a matter of fact, transformations of quantities that appear in (152)-(155) by the use of the rules (235) and (236) lead to the BBGKY chain with imaginary time

$$
\dot{\mathcal{D}}+6 \mathcal{H} \mathcal{D}+4 \dot{\mathcal{W}}_{1}+16 \mathcal{H} \mathcal{W}_{1}=0
$$




$$
\begin{aligned}
& \dddot{\mathcal{W}}_{n}+3(2 n+3) \mathcal{H} \ddot{\mathcal{W}}_{n} \\
& +3\left[\left(4 n^{2}+12 n+6\right) \mathcal{H}^{2}+(2 n+1) \dot{\mathcal{H}}\right] \dot{\mathcal{W}}_{n} \\
& +2 n\left[2\left(2 n^{2}+9 n+9\right) \mathcal{H}^{3}+6(n+2) \mathcal{H} \dot{\mathcal{H}}+\ddot{\mathcal{H}}\right] \mathcal{W}_{n} \\
& +4 \dot{\mathcal{W}}_{n+1}+8(n+2) \mathcal{H} \mathcal{W}_{n+1}=0, \quad n=1, \cdots, \infty,
\end{aligned}
$$

and to macroscopic Einstein's equations with imaginary time

$$
\begin{aligned}
& \dot{\mathcal{H}}=-\frac{1}{16} \mathcal{D}-\frac{1}{6} \mathcal{W}_{1}, \\
& 3 \mathcal{H}^{2}=\frac{1}{16} \mathcal{D}+\frac{1}{4} \mathcal{W}_{1} .
\end{aligned}
$$

It is easy to see that for $\Lambda=0$ Equations (152)-(155) identically coincide with (237)-(239) after some trivial renaming.

The invariance of the theory with respect to the Wick rotation of the time axis leads to the nontrivial consequence. Having only self-consistent solution of the $B B G K Y$ chain and macroscopic Einstein's equations, we can not say whether this solution is in real or imaginary time. Nevertheless, having a concrete solution of BBGKY chain, we can view the status of time during further study. To do so, it is necessary to explore the opportunity to obtain the same solution at the level of operator functions and state vectors. If this opportunity exists, the appropriate self-consistent solution of BBGKY chain and macroscopic Einstein's equations is recognized as existing in real time. In the previous Section 6, we showed that two exact solutions (186.I) and (186.III) really exist at the level of operators and vectors, and thus have a physical interpretation of standard notions of quantum theory.

The problem is: What a physical reality reflects the existence of solutions to Equations (152)-(155) (or that the same thing, (237)-(239)), not reproducible in real time at the level of operators and vectors? The existence of the problem is explicitly demonstrated by the example of exact solutions (186.II). Assume that this solution for $C_{3 g}=0, C_{4 g}=-k_{0}^{2}<0, C_{g 2}=3 k_{0}^{2} / 4$ exists in real time:

$$
H^{2}=\frac{k_{0}^{2}}{a^{2}} \ln \frac{a}{a_{0}}, \quad a=a_{0} \exp \left(\frac{k_{0}^{2} \eta^{2}}{4}\right) .
$$

The wave equation for gravitons with the (240) background reads

$$
\hat{\psi}_{\mathbf{k} \sigma}^{\prime \prime}+k_{0}^{2} \eta \hat{\psi}_{\mathbf{k} \sigma}^{\prime}+k^{2} \hat{\psi}_{\boldsymbol{k} \sigma}=0 .
$$

The equation for the ghosts looks similar. Equation (241) differs from (202) just in the sign of coefficient before the first derivative. However, this difference is crucial: if $k^{2} / k_{0}^{2}>0$ it is impossible to allocate the finite Hermit $H_{1}(\eta)$ polynomial from degenerate hypergeometric functions that correspond to solutions of Equation (241). We have been left with the infinite series only. These series and integrals over spectrum of products of these series can not be made consistent with the simple mathematical structure of the exact solutions (186.II). For this reason the solution (186.II), as the functional of scale factor is not relevant to solving operator equations in real time.

\subsubsection{Imaginary Time Formalism}

As is known, the imaginary time formalism is used in non-relativistic Quantum Mechanics (QM) (examples see, e.g., in book [43]), in the instanton theory of Quantum Chromodynamics (QCD) [44-49] and in the axiomatic quantum field theory (AQFT) (See Chapter 9 in the monograph [25]). The instanton physics in Quantum Cosmology was discussed in [51,52].

In QM and QCD the imaginary time formalism is a tool for the study of tunnelling, uniting classic independent states that are degenerate in energy, in a single quantum state. In AQFT, the Schwinger functions are defined in the four-dimensional Euclidian space-Euclid analogues of Wightman functions defined over the Minkowski space. It is believed that using properties of Euclid-Schwinger functions after their analytical continuation to the Minkowski space, one can reconstruct the properties of Wightman functions, and thereby restore the physical meaning of the appropriate model of quantum field theory.

All prerequisites for the use of the formalism of imaginary time in the QM and QCD on the one hand, and in AQFT, on the other hand, are united in the self-consistent theory of gravitons. Immediately, however, the specifics of the graviton theory under discussion should be noted. Macroscopic space-time in self-consistent theory of gravitons, unlike the space-time in the QM, QCD and AQFT, is a classical dynamic subsystem, which actually evolved in real time. If in QCD and AQFT Wick's turn is used to examine the significant properties of quantum system expressed in the probabilities of quantum processes, then in relation to the deterministic evolution of classical macroscopic subsystem this turn makes no sense. Therefore, after solving equations of the theory in imaginary time, we are obliged to apply (to the solution obtained) the operation of analytic continuation of the space for the positive signature to the space of negative signature. It is clear from the outset that the operation is not reduced to the opposite Wick turn, but is an independent postulate of the theory.

Before discussing the physical content of the theory, let us define its formal mathematical scheme. The theory is formulated in the space with metric

$$
d s^{2}=-d \tau^{2}-a^{2}(\tau)\left(d x^{2}+d y^{2}+d z^{2}\right) .
$$


Note that in our theory, that is suppose to do with cosmological applications (as opposed to QCD and AQFT), one of the coordinates is singled out simply because the scale factor depends on it. This means that in the classical sector of the theory time $\tau$, despite the fact that it is imaginary, is singled out in comparison with the 3 -spatial coordinates. In the quantum sector the $\tau$ coordinate also has a special status. Operators of graviton and ghost fields with nontrivial commutation properties are defined over the space (242). Symmetry properties of space (242) allow us to define the Fourier images of the operators by coordinates $x, y, z$, and to formulate the canonical commutation relations in terms of derivatives of operators with respect to the imaginary time $\tau$ :

$$
\begin{aligned}
& \frac{a^{3}}{4 \varkappa}\left[\frac{\mathrm{d} \hat{\psi}_{k \sigma}^{+}}{\mathrm{d} \tau}, \hat{\psi}_{k^{\prime} \sigma^{\prime}}\right]_{-}=-i \hbar \delta_{k k^{\prime}} \delta_{\sigma \sigma^{\prime}} \cdot \\
& \frac{a^{3}}{4 \varkappa}\left[\frac{\mathrm{d} \hat{\vartheta}_{k}^{+}}{\mathrm{d} \tau}, \hat{\vartheta}_{\boldsymbol{k}^{\prime}}\right]_{-}=-i \hbar \delta_{k k^{\prime}}, \\
& \frac{a^{3}}{4 \varkappa}\left[\frac{\mathrm{d} \hat{\vartheta}_{k}}{\mathrm{~d} \tau}, \hat{\vartheta}_{k^{\prime}}^{+}\right]_{-}=-i \hbar \delta_{k k^{\prime}} .
\end{aligned}
$$

Note that (243), (244) are introduced by the newly independent postulate of the theory, and not derived from standard commutation relations (83), (87) by conversion of $t \rightarrow i \tau$. (Such a conversion would lead to the disappearance of the imaginary unit from the right hand sides of the commutation relations.) Thus, the imaginary time formalism can not be regarded simply as another way to describe the graviton and ghost fields, i.e. as a mathematically equivalent way for real time description. In this formalism the new specific class of quantum phenomena is studied.

The system of self-consistent equations is produced by variations of action, as defined in 4-space with a positive signature:

$$
\begin{aligned}
S= & \frac{1}{\varkappa} \int \mathrm{d} \tau\left\{3\left[\frac{a^{2}}{N} \frac{\mathrm{d}^{2} a}{\mathrm{~d} \tau^{2}}-\frac{a^{2}}{N^{2}} \frac{\mathrm{d} N}{\mathrm{~d} \tau} \frac{\mathrm{d} a}{\mathrm{~d} \tau}+\frac{a}{N}\left(\frac{\mathrm{d} a}{\mathrm{~d} \tau}\right)^{2}\right]\right. \\
& +\frac{1}{8} \sum_{\boldsymbol{k} \sigma}\left(\frac{a^{3}}{N} \frac{\mathrm{d} \hat{\psi}_{\boldsymbol{k} \sigma}^{+}}{\mathrm{d} \tau} \frac{\mathrm{d} \hat{\psi}_{\boldsymbol{k} \sigma}}{\mathrm{d} \tau}+N a k^{2} \hat{\psi}_{\boldsymbol{k} \sigma}^{+} \hat{\psi}_{\boldsymbol{k} \sigma}\right) \\
& \left.-\frac{1}{4} \sum_{\boldsymbol{k}}\left(\frac{a^{3}}{N} \frac{\mathrm{d} \hat{\vartheta}_{k}^{+}}{\mathrm{d} \tau} \frac{\mathrm{d} \hat{\vartheta}_{k}}{\mathrm{~d} \tau}+N a k^{2} \hat{\vartheta}_{\boldsymbol{k}} \hat{\vartheta}_{k}\right)\right\} .
\end{aligned}
$$

Note that the full derivative with respect to the imaginary time is not excluded from Lagrangian. In (245) the integrand contains the density of invariant $\sqrt{\hat{g}} \hat{R}$. The Lagrange multiplier $N$ after the completion of the variation procedure is assumed to be equal to unity. The system of equations corresponding to the action (245) can also be obtained from the system of equations in real time by conversion of $t \rightarrow i \tau$. Quantum equations of motion for field operators in the imaginary time read

$$
\begin{aligned}
& \frac{\mathrm{d}^{2} \hat{\psi}_{\boldsymbol{k} \sigma}}{\mathrm{d} \tau^{2}}+3 \mathcal{H} \frac{\mathrm{d} \hat{\psi}_{\boldsymbol{k} \sigma}}{\mathrm{d} \tau}-\frac{k^{2}}{a^{2}} \hat{\psi}_{\boldsymbol{k} \sigma}=0, \\
& \frac{\mathrm{d}^{2} \hat{\vartheta}_{\boldsymbol{k}}}{\mathrm{d} \tau^{2}}+3 \mathcal{H} \frac{\mathrm{d} \hat{\vartheta}_{\boldsymbol{k}}}{\mathrm{d} \tau}-\frac{k^{2}}{a^{2}} \hat{\vartheta}_{\boldsymbol{k}}=0,
\end{aligned}
$$

where $\mathcal{H}=\dot{a} / a$.

Equations (246), (247) differ from (76), (77) by only replacement of $k^{2} \rightarrow-k^{2}$. At the level of analytic properties of solutions of the equations this difference, of course, is crucial. However, formal transformations, not dependent on the properties of analytic solutions to Equations (76), (77) and (246), (247), look quite similar. Therefore, all operations to construct the equation for the spectral function in imaginary time (analogue to Equation (149)) and the subsequent construction of BBGKY chain coincide with that described in Section 5.1 with the replacement of $k^{2} \rightarrow-k^{2}$. Replacing $k^{2} \rightarrow-k^{2}$ changes the definition of moments only parametrically: instead of (151) we get

$$
\begin{aligned}
& \mathcal{W}_{n}=\sum_{\boldsymbol{k}}\left(\frac{-k^{2}}{a^{2}}\right)^{n} \\
& \cdot\left(\sum_{\sigma}\left\langle\Psi_{g}\left|\hat{\psi}_{\boldsymbol{k} \sigma}^{+} \hat{\psi}_{\boldsymbol{k} \sigma}\right| \Psi_{g}\right\rangle-2\left\langle\Psi_{g h}\left|\hat{\vartheta}_{\boldsymbol{k}}^{+} \hat{\vartheta}_{\boldsymbol{k}}\right| \Psi_{g h}\right\rangle\right), \\
& n=0,1,2, \cdots, \infty, \\
& \mathcal{D}=\frac{\mathrm{d}^{2} \mathcal{W}_{0}}{\mathrm{~d} \tau^{2}}+3 \mathcal{H} \frac{\mathrm{d} \mathcal{W}_{0}}{\mathrm{~d} \tau} .
\end{aligned}
$$

Further actions lead obviously to the BBGKY chain (237), (238) and to the macroscopic Einstein equations (239).

To solve Equations (246) and (247), we will be using only the real linear-independent basis

$$
\begin{aligned}
& \hat{\psi}_{\boldsymbol{k} \sigma}=\sqrt{4 \varkappa \hbar}\left(\hat{Q}_{\mathbf{k} \sigma} g_{\boldsymbol{k}}+\hat{P}_{\boldsymbol{k} \sigma} h_{\mathbf{k}}\right), \\
& \hat{\vartheta}_{\boldsymbol{k}}=\sqrt{4 \varkappa \hbar}\left(\hat{q}_{\boldsymbol{k}} g_{\boldsymbol{k}}+\hat{p}_{\boldsymbol{k}} h_{\boldsymbol{k}}\right), \\
& g_{k} \dot{h}_{k}-h_{k} \dot{g}_{k}=\frac{1}{a^{3}} .
\end{aligned}
$$

As will be seen below, one of the basic solutions satisfies the known definition of instanton: an instanton is a solution to the classical equation, which is localized in the imaginary time and corresponds to the finite action in the 4-space with a positive signature. We will call the operator functions (249) the quantum instanton fields of gravitons and ghosts. Operator constants of integration $\hat{Q}_{k \sigma}, \hat{P}_{k \sigma}$ and $q_{k}, p_{k}$ satisfy commutation relations (101). Ladder operators are imposed by Equation (100) and then used in the procedure for constructing the state vectors over the basis of occupation numbers. State 
vectors of the general form in graviton and ghost sectors are already familiar structure (94) and (97). Only the interpretation of occupation numbers is changed: now it is number of instantons $n_{k \sigma}, n_{k}, \bar{n}_{k}$ of graviton, ghost and anti-ghost types, respectively.

Direct calculation of the moments of the spectral function leads to the expression:

$$
\mathcal{W}_{n}=4 \varkappa \hbar(-1)^{n} \sum_{k}\left(\frac{k^{2}}{a^{2}}\right)^{n}\left(A_{k} g_{k}^{2}+B_{k} h_{k}^{2}\right),
$$

where

$$
\begin{aligned}
A_{k}= & \sum_{\sigma}\left\langle\Psi_{g}\left|\hat{Q}_{\boldsymbol{k} \sigma}^{+} Q_{\mathbf{k} \sigma}\right| \Psi_{g}\right\rangle-2\left\langle\Psi_{g h}\left|\hat{q}_{\boldsymbol{k}}^{+} \hat{q}_{\boldsymbol{k}}\right| \Psi_{g h}\right\rangle \\
= & 2\left\langle n_{k(g)}\right\rangle\left(1+\zeta_{k}^{(g)} \cos \varphi_{k}\right) \\
& -2\left\langle n_{k(g h)}\right\rangle\left(1+\zeta_{k}^{(g h)} \cos \chi_{k}\right), \\
B_{k}= & \sum_{\sigma}\left\langle\Psi_{g}\left|\hat{P}_{\boldsymbol{k} \sigma}^{+} P_{\boldsymbol{k} \sigma}\right| \Psi_{g}\right\rangle-2\left\langle\Psi_{g h}\left|\hat{p}_{\boldsymbol{k}}^{+} \hat{p}_{\boldsymbol{k}}\right| \Psi_{g h}\right\rangle \\
= & 2\left\langle n_{k(g)}\right\rangle\left(1-\zeta_{k}^{(g)} \cos \varphi_{k}\right) \\
& -2\left\langle n_{k(g h)}\right\rangle\left(1-\zeta_{k}^{(g h)} \cos \chi_{k}\right) .
\end{aligned}
$$

The term containing products of basis functions $g_{k} h_{k}$ is eliminated from (250) by the condition of homogeneity of 3-space. In (251) and (252) average values of numbers of instantons of ghost and anti-ghost types are assumed to be equal: $\left\langle n_{k(g h)}\right\rangle=\left\langle\bar{n}_{k(g h)}\right\rangle$. One needs to pay attention to the multiplier $(-1)^{n}$ in (250): the alternating sign of moments is a common symptom of instanton nature of the spectral function.

Instanton equations of motion (246), (247) are of the hyperbolic type. This fact determines the form of asymptotics of basis function for $k|\xi| \gg 1$ where $\xi=\int \mathrm{d} \tau / a$ is conformal imaginary time. One of basis functions is localized in the imaginary time and the other is increasing without limit with the increasing of modulus of the imaginary time

$$
g_{k} \sim \frac{\mathrm{e}^{-k \xi}}{a \sqrt{2 k}}, \quad h_{k} \sim \frac{\mathrm{e}^{k \xi}}{a \sqrt{2 k}}, \quad k|\xi| \gg 1 .
$$

In this situation, it is necessary to differentiate between stable and unstable instanton configurations. We call a configuration stable, if moments of the spectral function are formed by localized basis functions only. Without limiting generality, we assigned $h_{k}$ to the class of increasing functions. It is easy to see that the condition of stability $B_{k}=0$ that eliminates contributions of $h_{k}$ from (250) is reduced to the condition of quantum coherence of instanton condensate:

$$
\begin{aligned}
& \zeta_{k}^{(g)} \cos \varphi_{k}=\zeta_{k}^{(g h)} \cos \chi_{k}=1 \\
& \rightarrow \zeta_{k}^{(g)}=\zeta_{k}^{(g h)}=1, \quad \cos \varphi_{k}=\cos \chi_{k}=1 .
\end{aligned}
$$

Expressions for the moments are simplified and read

$$
\mathcal{W}_{n}=4 \varkappa \hbar(-1)^{n} \sum_{k} A_{\boldsymbol{k}}\left(\frac{k^{2}}{a^{2}}\right)^{n} g_{k}^{2} .
$$

Exact solutions, with the stable instanton configurations, are described in the following Sections 7.2 and 7.3. In principle, for a limited imaginary time interval, there might be unstable configurations, but in the present work such configurations are not discussed. (The example of the unstable instanton configuration see in [53]).

Note that moments (255) can be obtained within the classical theory, limited, as generally accepted, to the solutions localized in imaginary time. In doing so, $A_{k}$ acts as a constant of integration of classical equation.

The above approach is the quantum theory of instantons in imaginary time. Here are present all the elements of quantum theory: operator nature of instanton field; quantization on the canonical commutation relations; basic vectors in the representation of instanton occupation numbers; state vectors of physical states in the form of superposition of basic vectors. With the quantum approach, a significant feature of instantons is displayed, which clearly is not visible in the classical theory. It is the nature of instanton stable configurations as coherent quantum condensates.

Construction of the formalism of the theory is completed by developing a procedure to transfer the results of the study of instantons to real time. It is clear that this procedure is required to match the theory with the experimental data, i.e. to explain the past and predict the future of the Universe. As already noted, the procedure of transition to real time is not an inverse Wick rotation. This is particularly evident in the quantum theory: in (243), (244) the reverse Wick turn leads to the commutation relations for non-Hermitian operators, which can not be used to describe the graviton field.

The procedure for the transition to real time has the status of an independent theory postulates. We will formulate this postulate as follows.

1) Results of solutions of quantum equations of motion (246), (247), together with the macroscopic Einstein's Equation (239) after calculating of the moments (that is, after averaging over the instanton state vector) should be represented in the functional form

$$
\mathcal{D}=\mathcal{D}(a, \mathcal{H}, \dot{\mathcal{H}}, \cdots), \quad \mathcal{W}_{n}=\mathcal{W}_{n}(a, \mathcal{H}, \dot{\mathcal{H}}, \cdots) .
$$

2) It is postulated that functional dependence of the moments of the spectral function on functions describing the macroscopic geometry must be identical in the real and imaginary time. Thus, at the level of the moments of the spectral function, the transition to the real time is reduced to a change of notation 


$$
\begin{aligned}
& \mathcal{D}(a, \mathcal{H}, \dot{\mathcal{H}}, \cdots) \rightarrow D(a, H, \dot{H}, \cdots), \\
& \mathcal{W}_{n}(a, \mathcal{H}, \dot{\mathcal{H}}, \cdots) \rightarrow W_{n}(a, H, \dot{H}, \cdots) .
\end{aligned}
$$

3) Moments $D(a, H, \dot{H}, \cdots)$ and $W_{1}(a, H, \dot{H}, \cdots)$ obtained by operations (257), are substituted to right hand side of macroscopic Einstein equations that are considered now as equations in real time. Formally this means that the transition to the real time in the left hand side of Equation (239) is reduced to changing of the following notations

$$
\dot{\mathcal{H}} \rightarrow \dot{H}, \quad \mathcal{H}^{2} \rightarrow H^{2} .
$$

Thus, the acceptance of postulates (256)-(258) is equivalent to the suggestion that in real time the self-consistent evolution of classic geometry and quantum instanton system is described by the following equations

$$
\begin{aligned}
& \dot{H}=-\frac{1}{16} D(a, H, \dot{H}, \cdots)-\frac{1}{6} W_{1}(a, H, \dot{H}, \cdots), \\
& 3 H^{2}=\frac{1}{16} D(a, H, \dot{H}, \cdots)+\frac{1}{4} W_{1}(a, H, \dot{H}, \cdots),
\end{aligned}
$$

under the condition that the form of functionals in right hand sides of (259) is established by microscopic calculations in imaginary time. It is obvious also that in the framework of these postulates any solution of equations consisting of BBGKY chain and macroscopic Einstein equations (obtained without use of microscopic theory) can be considered as the solution in real time.

\subsubsection{Physics of Imaginary Time}

Mathematical and physical motivation to look for the formalism of imaginary time comes from the fact that there are degenerate states separated by the classical impenetrable barrier. In non-relativistic quantum mechanics the barriers are considered, that have been formed by classical force fields and for that reason they have the obvious interpretation. It is well known, that the calculation of quantum tunnelling across the classical impenetrable barrier can be carried out in the following order: 1) the solution of classical equation of motion inside the barrier area is obtained with imaginary time; 2) from the solution obtained for the tunnelling particle, one calculates the action $S$ for the imaginary time; 3) the tunnelling probability, coinciding with the result of the solution for Schrodinger equation in the quasi-classical approximation, is equal $w=\mathrm{e}^{-S}$. Obviously, the sequence described bears a formal character and cannot be interpreted operationally. Nevertheless, a strong argument toward the use of the formalism of imaginary time in the quantum mechanics is the agreement between the calculations and experimental data for the tunnelling micro-particles.

A new class of phenomena arises in the cases when tunnelling processes form a macroscopic quantum state. The Josephson effect is a characteristic example: fluctuations of the electromagnetic field arise when a superconductive condensate is tunnelling across the classically impenetrable non-conducting barrier. Here, the tunnelling can be formally described as a process developing in imaginary time, but the fluctuations arise and exist in the real space-time. Experimental data show that regardless of the description, the tunnelling process forms a physical subsystem in the real space-time, with perfectly real energy-momentum.

In Quantum Chromodynamics (QCD) physically similar phenomena are studied by similar methods [49]. The vacuum degeneration is an internal property of QCD: different classical vacuums of gluon field are not topologically equivalent. In the framework of the classic dynamics any transitions between different vacuums are impossible. In that sense the topological non-equivalence plays role of the classical impenetrable barrier. There is an heuristic hypothesis in quantum theory-that the probability of tunnelling transition between different vacuums can be calculated as $w=e^{-S}$, where $S$ is the action of the classical instanton. The instanton is defined as a solution of gluon-dynamic equations localized in the Euclidian space-time connecting configurations with different topologies. As in the case of Josephson Effect, it is assumed that the tunnelling processes between topologically non-equivalent vacuums are accompanied by generation of non-perturbative fluctuations of gluon and quark fields in real space-time. Let us notice that in $Q C D$ the instanton solutions, analytically continued into real space-time, are used to evaluate the amplitude of fluctuations. The fluctuations in real space-time are considered as a quark-gluon condensate (QGC). The existence of QGC with different topological structure in "off-adrons" and "in-adrons" vacuums, is confirmed by comparison of theoretical predictions with experimental data. One of remarkable facts is that the carrier of approximately the half of nucleon mass is in fact the energy of the reconstructed QGC.

Now let us go back to the self-consistence theory of gravitons. In that theory, due to its one-loop finiteness, all observables are formed by the difference between graviton and ghost contributions. That fact is obvious both from the general expressions for the observables (see (118), (119)), and from the exact and approximate solutions (described in the previous sections) as well. The same final differences of contributions may correspond to the totally different graviton and ghost contributions themselves. All quantum states are degenerated with respect to mutually consistent transformations of gravitons and ghosts occupation numbers, but providing unchanged values of observable quantities. Thus the multitude of state vectors of the general form, averaging 
over which leads to the same values of spectral function, is a direct consequence of the internal mathematical structure of the self-consistent theory of gravitons, satisfying the one-loop finiteness condition.

In that situation, it is very natural to introduce a hypothesis about the tunnelling of the graviton-ghost system between quantum states corresponding to the same values of macroscopic observables. By the analogy with the effects described above, one may suggest that 1) the tunnelling processes unite degenerate quantum states into a single quantum state; 2) tunnelling is accompanied by creation of specific quantum fluctuations of graviton and ghost fields in real space-time. With regard to the mathematical method used to describe these phenomena, today we may use only those methods that have been tested in adjacent brunches of quantum theory. It is easy to see that this program has been realized in Sections 7.1.1 and 7.1.2. We solve the equations of the theory for imaginary time, but the amplitude of the arising fluctuations we evaluate by the analytical continuation (256)(258), analoguos to the ones used in QCD. The specific of our theory lie in the fact that at the final step of calculations we use the classical Einstein equation (259) describing the evolution of the macroscopic space in real time. The possibility of using these equations is determined by the action (245), which, when calculated by means of the instanton solutions and averaged over the state vector of instantons, is identically equal zero. As a matter of fact, after using instanton Equations (246) and (247) and averaging, the action (245) is reduced to the form:

$$
\langle\Psi|S| \Psi\rangle=\frac{1}{\varkappa} \int \mathrm{d} \tau a^{3}\left[3\left(\dot{\mathcal{H}}+\mathcal{H}^{2}\right)+\frac{1}{16} \mathcal{D}\right] .
$$

The integrand in (260) is equal zero in the Einstein equations with imaginary time (239). The fact that $w=\exp (-\langle\Psi|S| \Psi\rangle)=1$ means that the macroscopic evolution of the Universe is determined. That feature allows the use of Equation (259), after the moments are analytically continued into the real time.

\subsection{Instanton Condensate in the De Sitter Space}

Among exact solutions of the one-loop quantum gravity, a special status is given to De Sitter space if the space curvature of this space is self-consistent with the quantum state of gravitons and ghosts. In Section 6.2, it was shown that in the self-consistent solution, gravitons and ghosts can be interpreted as quantum wave fields in real space-time. Nevertheless, it should be mentioned, that the alternating sign of the moments (228) points to a possibility of instanton interpretation of that solution. Methods described in Sections 7.1.1 and 7.1.2, when applied to De Sitter space, show that such interpretation is really possible.
We will work with the imaginary conformal time $\xi=\int \mathrm{d} \tau / a$. The cosmological solution is:

$$
a=a_{0} \mathrm{e}^{\mathcal{H} \tau}=-\frac{1}{\mathcal{H} \xi}, \quad-\infty<\xi \leq 0 .
$$

At the level of the BBGKY chain, due to the fact that the theory is invariant with respect to the Wick rotation, the calculations performed to get the solutions coincide with the those described in Section 5.3. At the microscopic level we use the exact solutions (246), (247) with the background (261):

$$
\begin{aligned}
& \hat{\psi}_{\boldsymbol{k} \sigma}=\frac{1}{a} \sqrt{\frac{2 \varkappa \hbar}{k}}\left[Q_{\mathbf{k} \sigma} g(x)+P_{\boldsymbol{k} \sigma} h(x)\right], \\
& \hat{\vartheta}_{\boldsymbol{k}}=\frac{1}{a} \sqrt{\frac{2 \varkappa \hbar}{k}}\left[q_{\boldsymbol{k}} g(x)+p_{\boldsymbol{k}} h(x)\right],
\end{aligned}
$$

where $x=k \xi<0$,

$$
g(x)=\left(1-\frac{1}{x}\right) \mathrm{e}^{x}, \quad h(x)=\left(1+\frac{1}{x}\right) \mathrm{e}^{-x} .
$$

The expressions for the moments of the spectral function are reduced to the form:

$$
\mathcal{W}_{n}=(-1)^{n} \frac{\varkappa \hbar}{\pi^{2}} \mathcal{H}^{2 n+2} \int_{-\infty}^{0} \mathrm{~d} x x^{2 n+2}\left(A_{k} g^{2}+B_{k} h^{2}\right) .
$$

Equations for $A_{k}, B_{k}$ are given in (251), (252). From (263) it is obvious that the self-consistent values $\mathcal{W}_{n}=$ const can be obtained only for a flat spectrum of instantons. However, with the flat specter and $B_{k} \neq 0$, the second term in (263) creates a meaningless infinity. Therefore $B_{k}=0$, and that, in turn, leads to the condition (254), i.e. to quantum coherence of the instanton condensate. The quantitative characteristics of the condensate are formed by instantons only, localized in imaginary time.

It is easy to calculate of the converging integrals in (263):

$$
\begin{aligned}
& \int_{-\infty}^{0} \mathrm{~d} x x^{2 n+2}\left(1-\frac{1}{x}\right)^{2} \mathrm{e}^{2 x} \\
& =\frac{1}{2^{2 n+1}}(2 n-1) ! !(2 n+1)(n+2) .
\end{aligned}
$$

After analytical continuation into the real space-time, following the rules (256)-(258), we obtain the final result:

$$
\begin{aligned}
D= & -\frac{12 \varkappa \hbar N_{\text {inst }}}{\pi^{2}} H^{4}, \\
W_{n}= & \frac{(-1)^{n+1}}{2^{2 n}}(2 n-1) !(2 n+1)(n+2) \\
& \times \frac{2 \varkappa \hbar N_{\text {inst }}}{\pi^{2}} H^{2 n+2}, \quad n \geq 1
\end{aligned}
$$


where

$$
N_{i n s t}=\left\langle n_{g h}\right\rangle-\left\langle n_{g}\right\rangle
$$

The comparison of the two models of graviton-ghost condensate in the De Sitter space reveals some interesting features. In both cases we deal with the effect of quantum coherence. Expressions (265) differ from (228) only in the formal substitution $N_{g} \rightarrow N_{\text {inst }}$. However the conditions leading to the quantum coherence are different in these models. According to (229), in the condensate of virtual gravitons and ghosts, the average value of graviton and ghost occupational numbers are the same, and the non-zero effect appears due to the fact that the phase correlation in the quantum superposition in the graviton's and ghost's sectors are formed differently. As it follows from (254), (266), in the instanton condensate the phases in the graviton and ghost sectors correlate similarly, but the non-zero effect appears due to the difference of average occupation numbers for graviton's and ghost's instantons. The absence of the macroscopic structure of the condensates does not allow the detection of the differences by macroscopic measurements. In both cases the graviton-ghost vacuum possess equal energy-momentum characteristics.

The question about the actual nature of the De Sitter space is lies in the formal mathematical domain. In these circumstances one should pay attention to the following facts. While describing the condensate of virtual gravitons and ghosts, we were forced to introduce an additional definition of the mathematically non-existent integrals (225), i.e. to introduce into the theory some operations that were not present from the beginning. It is the additional operations that have provided a very specific property of the solution - the alternating signs in the sequence of the moments of the spectral function. By contrast, the theory of the instanton condensate has a completely different formal mathematics. The theory is motivated by the concrete property of the graviton-ghost system which is degeneration of quantum states, and the construction of the theory is constructed by the introduction of mathematically non-contradictory postulates. The moments of the spectral function's with alternating signs is an internal property of the graviton-ghost instanton theory. When we considered the instanton condensate in the De Sitter space, no additional mathematical redefinitions were necessary (compare the formulas (225) and (264)). We have the impression that the instanton version of the De Sitter space is more mathematically comprehensive. Therefore, one may suggest that the key role in the formation of the De Sitter space (the asymptotic state of the Universe) belongs to the instanton condensate, appearing in the tunnelling processes between degenerated states of the graviton-ghost vacuum $^{8}$.

\subsection{Instanton Condensate of Constant Conformal Wavelength}

The exact solution (186.II) has a pure instanton nature. Now we will obtain that solution with the value $C_{3 g}=0$. One can rewrite the formulas (240), (241) for the imaginary time:

$$
\begin{aligned}
& \mathcal{H}^{2}=\frac{k_{0}^{2}}{a^{2}} \ln \frac{a}{a_{0}}, \quad a=a_{0} \exp \left(\frac{k_{0}^{2} \xi^{2}}{4}\right) . \\
& \frac{\mathrm{d}^{2} \hat{\psi}_{\boldsymbol{k} \sigma}}{\mathrm{d} \xi^{2}}+k_{0}^{2} \xi \frac{\mathrm{d} \hat{\psi}_{\boldsymbol{k} \sigma}}{\mathrm{d} \xi}-k^{2} \hat{\psi}_{\boldsymbol{k} \sigma}=0, \\
& \frac{\mathrm{d}^{2} \hat{\vartheta}_{\boldsymbol{k}}}{\mathrm{d} \xi^{2}}+k_{0}^{2} \xi \frac{\mathrm{d} \hat{\vartheta}_{\boldsymbol{k}}}{\mathrm{d} \xi}-k^{2} \hat{\vartheta}_{\boldsymbol{k}}=0 .
\end{aligned}
$$

As we already know, the spatially homogeneous modes participate in the formation of the solution for the Equation (186.II). As follows from (268), when $k^{2} \rightarrow 0$, the description of the spatially homogeneous modes in imaginary time does not differ from their description in real time. The contribution from modes $g 2$ is present in (267), with the relations $C_{g 4}=-k_{0}^{2}<0, C_{g 2}=3 k_{0}^{2} / 4$ taken into account. These relations are necessary to provide the existence of the self-consistent solution. In what follows we are considering the quasi-resonant modes only.

For $k^{2}=k_{0}^{2}$, the signs of the last terms in the Equation (268) provide the existence of instanton solutions we are looking for:

$$
\begin{aligned}
& \hat{\psi}_{\boldsymbol{k} \sigma}=\frac{\sqrt{4 \varkappa \hbar k_{0}}}{a_{0}} \\
& \cdot\left[\xi\left(\hat{Q}_{\boldsymbol{k} \sigma}+k_{0} \hat{P}_{\boldsymbol{k} \sigma} \int_{0}^{\xi} \mathrm{e}^{-k_{0}^{2} \xi^{2} / 2} \mathrm{~d} \xi\right)+\frac{\hat{P}_{\boldsymbol{k} \sigma}}{k_{0}} \mathrm{e}^{-k_{0}^{2} \xi^{2} / 2}\right] \\
& =\sqrt{\frac{16 \varkappa \hbar}{k_{0} a_{0}^{2}}}\left[\hat{Q}_{\boldsymbol{k} \sigma}+\hat{P}_{\boldsymbol{k} \sigma} F(a)\right] \ln ^{1 / 2} \frac{a}{a_{0}}, \\
& \hat{\vartheta}_{k}=\frac{\sqrt{4 \varkappa \hbar k_{0}}}{a_{0}} \\
& \cdot\left[\xi\left(\hat{q}_{\boldsymbol{k}}+k_{0} \hat{p}_{\boldsymbol{k}} \int_{0}^{\xi} \mathrm{e}^{-k_{0}^{2} \xi^{2} / 2} \mathrm{~d} \xi\right)+\frac{\hat{p}_{\boldsymbol{k}}}{k_{0}} \mathrm{e}^{-k_{0}^{2} \eta^{2} / 2}\right] \\
& =\sqrt{\frac{16 \varkappa \hbar}{k_{0} a_{0}^{2}}}\left[\hat{q}_{\boldsymbol{k}}+\hat{p}_{\boldsymbol{k}} F(a)\right] \ln ^{1 / 2} \frac{a}{a_{0}},
\end{aligned}
$$

\footnotetext{
${ }^{8} \mathrm{~A}$ cosmological scenario based on this solution was proposed in [50]. In this scenario, birth of the flat inflationary Universe can be thought of as a quantum tunneling from "nothing". As the Universe ages and is emptied, the same mechanism of tunneling that gave rise to the empty Universe at the beginning, gives now birth to dark energy. The emptying Universe should possibly complete its evolution by tunneling back to "nothing".
} 
where

$$
F(a)=a_{0}^{2} \int_{a_{0}}^{a} \frac{\mathrm{d} a}{a^{3} \ln ^{1 / 2} \frac{a}{a_{0}}}+\frac{a_{0}^{2}}{2 a^{2} \ln ^{1 / 2} \frac{a}{a_{0}}} .
$$

Calculations which follow contain the same mathematical operations we have already described several times in the previous sections. After we remove contributors to the spectral function which contains $F(a)$, we obtain the condition for the coherence of the condensate. Some details of the calculations is related to the alternating signs of the moments, i.e. with the multiplier $(-1)^{n}$, characteristic for the instanton theory. Particularly, in the expression for $\mathcal{W}_{1}(g 4)$, there is a general sign "minus" . But, according to the Einstein equations in imaginary time $\mathcal{W}_{1}(g 4)>0$. The positive sign of the first moment is provided by the dominant contribution of ghost instantons over the contribution of graviton instantons. With that taken into account, we obtain the final equations for the moments of quasi-resonant modes, obtained after the analytic continuation into the real space-time:

$$
\begin{aligned}
W_{n}(g 4) & =(-1)^{n+1} \frac{64 \varkappa \hbar N_{\text {inst }}^{(g h)} k_{0}^{2 n-1}}{a_{0}^{2} a^{2 n}} \ln \frac{a}{a_{0}} \\
& =(-1)^{n+1} \frac{24 k_{0}^{2 n}}{a^{2 n}} \ln \frac{a}{a_{0}}, \\
D(g 4) & =-\frac{128 \varkappa \hbar N_{\text {inst }}^{(g h)} k_{0}}{a_{0}^{2} a^{2}} \ln \frac{\mathrm{e}^{1 / 4} a}{a_{0}} \\
& =-\frac{48 k_{0}^{2}}{a^{2}} \ln \frac{\mathrm{e}^{1 / 4} a}{a_{0}} .
\end{aligned}
$$

Here the following definition has been used:

$$
\begin{aligned}
& \left\langle n_{k(g h)}\right\rangle-\left\langle n_{k(g)}\right\rangle \rightarrow \frac{2 \pi^{2}}{k^{2}} N_{i n s t}^{(g h)} \delta\left(k-k_{0}\right), \\
& N_{\text {inst }}^{(g h)}=\frac{3 k_{0} a_{0}^{2}}{8 \varkappa \hbar} .
\end{aligned}
$$

The graviton instantons are dominant for the spatially homogeneous modes:

$$
\begin{aligned}
& W_{1}(g 2)=\frac{16 \varkappa \hbar k_{1} N_{i n s t}^{(g)}}{a_{1}^{2} a^{2}}, \\
& D(g 2)=-\frac{32 \varkappa \hbar k_{1} N_{\text {inst }}^{(g)}}{a_{1}^{2} a^{2}} .
\end{aligned}
$$

The parameter of the spatially homogeneous condensate is defined as follows:

$$
\begin{aligned}
& \left\langle n_{0(g)}\right\rangle\left(1+\zeta_{0}^{(g)} \cos \varphi_{0}\right)-\left\langle n_{0(g h)}\right\rangle\left(1+\zeta_{0}^{(g h)} \cos \chi_{0}\right) \\
& \rightarrow \frac{2 \pi^{2}}{k^{2}} N_{i n s t}^{(g)} \delta\left(k-q_{0}\right), \quad q_{0} \rightarrow 0 .
\end{aligned}
$$

From Expressions (271) and (272), one gets energy density and pressure for the system of quasi-resonant and spatially homogeneous instantons:

$$
\begin{aligned}
\varkappa \varepsilon_{g}= & \frac{8 \varkappa \hbar k_{0} N_{\text {inst }}^{(g h)}}{a_{0}^{2} a^{2}} \ln \frac{a}{a_{0}} \\
& +\frac{2 \varkappa \hbar}{a^{2}}\left(\frac{k_{0} N_{\text {inst }}^{(g h)}}{a_{0}^{2}}-\frac{k_{1} N_{\text {inst }}^{(g)}}{a_{1}^{2}}\right) \\
& =\frac{8 \varkappa \hbar k_{0} N_{\text {inst }}^{(g h)}}{a_{0}^{2} a^{2}} \ln \frac{a}{a_{0}}, \\
\varkappa p_{g} & =-\frac{8 \varkappa \hbar k_{0} N_{\text {inst }}^{(g h)}}{3 a_{0}^{2} a^{2}} \ln \frac{\mathrm{e} a}{a_{0}} \\
& -\frac{2 \varkappa \hbar}{3 a^{2}}\left(\frac{k_{0} N_{\text {inst }}^{(g h)}}{a_{0}^{2}}-\frac{k_{1} N_{\text {inst }}^{(g)}}{a_{1}^{2}}\right) \\
= & -\frac{8 \varkappa \hbar k_{0} N_{\text {inst }}^{(g h)}}{3 a_{0}^{2} a^{2}} \ln \frac{\mathrm{e} a}{a_{0}} .
\end{aligned}
$$

In Formula (273), the terms in brackets are eliminated by the condition (160), which is rewritten in terms of macroscopic parameters

$$
\frac{k_{0} N_{\text {inst }}^{(g h)}}{a_{0}^{2}}=\frac{k_{1} N_{\text {inst }}^{(g)}}{a_{1}^{2}} .
$$

Solutions (273), (274) describe a quantum coherent condensate of quasi-resonant instantons with the ghost dominance. The parameters of the condensate are in accordance with parameters of a spatially homogeneous condensate with graviton dominance.

\section{Discussion}

From the formal mathematical point of view, the above theory is identical to transformations of equations, determined by the original gauged path integral (1), leading to exact solutions for the model of self-consistent theory of gravitons in the isotropic Universe. To assess the validity of the theory, it is useful to discuss again but briefly the three issues of the theory that are missing in the original path integral.

1) The hypothesis of the existence of classic spacetime with deterministic, but self-consistent geometry is introduced into the theory. It is not necessary to discuss in detail this hypothesis because it simply reflects the obvious experimental fact (region of Planck curvature and energy density is not a subject of study in the theory under discussion). Note, however, that the introduction of this hypothesis into the formalism of the theory leads to a rigorous mathematical consequence: the strict definition of the operation of separation of classical and quantum variables uniquely captures the exponential parameterization of the metric. 
2) The transfer to the one-loop approximation is conducted in the self-consistent classical and quantum system of equations. Formally, this approximation is of a technical nature because the equations of the theory are simplified only in order to obtain specific approximate solutions. After classical and quantum variables are identified, the procedure of transition to the one-loop approximation is of a standard and known character [17]. In reality, of course, the situation in the theory is much more complex and paradoxical. On the one hand, the quantum theory of gravity is a non-renormalized theory (see e.g. [2]). Specific quantitative studies of effects off one-loop approximation are simply impossible. On the other hand, the quantum theory of gravity without fields of matter is finite in the one-loop approximation [3]. The latter means that the results obtained in the framework of one-loop quantum gravity pose limits to its applicability that is mathematically clear and physically significant. The existence of a range of validity for the one-loop quantum gravity without fields of matter is a consequence of two facts. First, there are supergravity theories with fields of matter which are finite beyond the limits of one-loop approximation. Second, the quantum graviton field is the only physical field with a unique combination of such properties as conformal non-invariance and zero rest mass. For this field only there is no threshold for the vacuum polarization and particle creation in the isotropic Universe. Therefore, in the stages of evolution of the Universe, where

$H^{2},|\dot{H}| \ll m^{2}$ ( $m$ is mass of any of the elementary particles), quantum gravitational effects can occur only in the subsystem of gravitons. It is also clear that in any future theory that unifies gravity with other physical interactions, equations of theory of gravitons in one-loop approximation will not be different from those we discuss in this work. Therefore the self-consistent theory of gravitons has the right to lay claim be a reliable description of the most significant quantum gravity phenomena in the isotropic Universe.

3) The need to use the Hamilton gauge, which provides a transition from the path integral to the Heisenberg representation [19], and then to the self-consistent theory of gravitons in the macroscopic spacetime. It is important that in the Hamilton gauge the dynamic properties of the ghost fields automatically provide one-loop finiteness of the theory off mass shell of gravitons and ghosts. The condition of one-loop finiteness off the mass shell largely determines the mathematical and physical content of the theory. Given that the main results of this work are exact solutions and exact transformations, the evaluation of he proposed approach is reduced to a discussion of this point of the theory. Let us enumerate once more logical and mathematical reasons, forcing us to include the condition of one-loop finiteness off the mass shell into the structure of the theory.

a) Future theory that will unify quantum gravity with the theory of other physical interactions may not belong to renormalizability theories. If such a theory exists, it may only be a finite theory. One-loop finiteness of quantum gravity with no fields of matter that is fixed on the mass shell [3] can be seen as the prototype of properties of the future theory.

b) Because of their conformal non-invariance and zero rest mass, gravitons and ghosts fundamentally can not be located exactly on the mass shell in the real Universe. Therefore, the problem of one-loop finiteness off the mass shell is contained in the internal structure of the theory.

c) In formal schemes, which do not meet the one-loop finiteness, divergences arise in terms of macroscopic physical quantities. To eliminate these divergences, one needs to modify the Lagrangian of the gravity theory, entering quadratic invariants. This, in turn, leads to abandonment of the original definition of the graviton field that generates these divergences. The logical inconsistency of such a formal scheme is obvious. (The mathematical proof of this claim is contained at Section 10.2.)

d) In the self-consistent theory of gravitons, one-loop finiteness off the mass shell can be achieved only through mutual compensation of divergent graviton and ghost contributions in macroscopic quantities. The existence of gauges, automatically providing such a compensation, is an intrinsic property of the theory.

From our perspective, the properties of the theory identified in points a), b), c) and d), clearly dictate the need to use only the formulation of self-consistent theory of gravitons, in which the condition of one-loop finiteness off the mass shell (the condition of internal consistency of the theory) is performed automatically. We also want to emphasize that, as it seems to us, the scheme of the theory given below has no alternative both logically and mathematically.

Ganged path integral $\Rightarrow$ choosing the Hamilton gauge, which provides one-loop finiteness of the theory off mass shell of gravitons and ghosts $\Rightarrow$ factorization of classic and quantum variables, which ensures the existence of a self-consistent system of equations $\Rightarrow$ transition to the one-loop approximation, taking into account the fundamental impossibility of removing the contributions of ghost fields to observables - appears to us logically and mathematically as the only choice.

As part of the theories preserving macroscopic spacetime being clearly one of its components, we see two topics for further discussions. The first of these is the replication of the results of this work by mathematically equivalent formalisms of one-loop quantum gravity. Here we can note that, for example, in the formalism of the 
extended phase space with BRST symmetry, our results are reproduced, even though the mathematical formalism is more cumbersome. The second topic is the reproduction of our results in more general theories than the oneloop quantum gravity without fields of matter. Here is meant a step beyond the limits of one-loop approximation as well as a description of quantum processes involving gravitons, while taking into account the existence of other quantum fields of spin $J \leq 3 / 2$. In the framework of discussion on this topic, we can make only one assertion: in the one-loop $N=1$ supergravity containing graviton field and one gravitino field, the results of our work are fully retained. This is achieved by two internal properties of $N=1$ supergravity: 1) The sector of gravitons and graviton ghosts in this theory is exactly the same as in the one-loop quantum gravity without fields of matter; 2) The physical degrees of freedom of gravitino with chiral $h= \pm 3 / 2$ in the isotropic Universe are dynamically separated from the non-physical degrees of freedom and are conformally invariant; 3) The gauge of gravitino field can be chosen in such a way that the gravitino ghosts automatically provide one-loop finiteness of $N=1$ supergravity. As for multi-loop calculations in the $N=1$ supergravity and more advanced theoretical models, we have not explored the issue.

Of course, a rather serious problem of the physical nature of ghosts remains. The present work makes use in practice only of formal properties of quantum gravity of Faddeev-Popov-De Witt, which point to the impossibility in principle of removing contributions of ghosts to observable quantities off the mass shell. A deeper analysis undoubtedly will address the foundations of quantum theory. In particular, one should point out the fact that the formalism of the path integral of Faddeev-Popov-De Witt is mathematically equivalent to the assumption that observable quantities can be expressed through derivatives of operator-valued functions defined on the classical spacetime of a given topology. On the other hand, finiteness of physical quantities is ensured in the axiomatic quantum field theory by invoking limited field operators smoothed over certain small areas of spacetime. Extrapolation of this idea to quantum theory of gravity immediately brings up the question on the role of spacetime foam [18] (fluctuations of topology on the microscopic level) in the formation of smoothed operators, and consequently, observable quantities. To make this problem more concrete, a question can be posed on collective processes in a system of topological fluctuations that form the foam. It is not excluded that the non-removable Faddeev-Popov ghosts in ensuring the one-loop finiteness of quantum gravity are at the same time a phenomenological description of processes of this kind.

Study of equations of self-consistent theory of gravitons, automatically satisfying the condition of one-loop finiteness, leads to the discovery of a new class of physical phenomena which are macroscopic effects of quantum gravity. Like the other two macroscopic quantum phenomena of superconductivity and superfluidity, macroscopic effects of quantum gravity occur on the macroscopic scale of the system as a whole, in this case, on the horizon scale of the Universe. Interpretation of these effects is made in terms of gravitons-ghost condensates arising from the interference of quantum coherent states. Each of coherent states is a state of gravitons (or ghosts) with a certain wavelength of the order of the distance to the horizon and a certain occupation number. The vector of the physical state is a coherent superposition of vectors with different occupation numbers.

A key part in the formalism of self-consistent theory of gravitons is played by the BBGKY chain for the spectral function of gravitons, renormalized by ghosts. It is important that equations of the chain may be introduced at an axiomatic level without specifying explicitly field operators and state vectors. It is only necessary to assume the preservation of the structure of the chain equations in the process of elimination of divergences of the moments of the spectral function. Three exact solutions of oneloop quantum gravity are found in the framework of BBGKY formalism. The invariance of the theory with respect to the Wick rotation is also shown. This means that the solutions of the chain equations, in principle, cover two types of condensates: condensates of virtual gravitons and ghosts and condensates of instanton fluctuations.

All exact solutions, originally found in the BBGKY formalism, are reproduced at the level of exact solutions for field operators and state vectors. It was found that exact solutions correspond to various condensates with different graviton-ghost microstructure. Each exact solution we found is compared to a phase state of graviton-ghost medium; quantum-gravity phase transitions are introduced.

We suspect that the manifold of exact solutions of one-loop quantum gravity is not exhausted by three solutions described in this paper. Search for new exact solutions and development of algorithms for that search, respectively, is a promising research topic within the proposed theory. Of great interest will also be approximate solutions, particularly those that describe non-equilibrium and unstable graviton-ghost and instanton configurations.

\section{Conclusions}

1) The equations of quantum gravity in the Heisenberg representation and the equations of semi-quantum/semiclassical self-consistent theory of gravitons in the macroscopic Riemann space, respectively, can exist only in the exponential parameterization and Hamilton gauge of 
the density of the contravariant metric;

2) Equations of semi-quantum/semi-classical theory necessarily contain the ghost sector in the form of a complex scalar field providing one-loop finiteness to the theory;

3) In case of isotropic Universe, in one-loop approximation the theory can be presented as a set of equations including Einsteins equations for the macroscopic metric with the energy-momentum tensor for gravitons and ghosts and BBGKY chain for the moments of the spectral function of gravitons renormalized by ghosts. Three exact solutions to the set of these equations are obtained which describe the various states of the graviton-ghost substratum;

4) Each exact solution to the BBGKY chain put in correspondence to the exact solutions of operator equations and observables averaged over the Heisenberg state vector. It was found that various exact solutions describe various graviton, ghost and instanton condensates on the horizon scale of the Universe;

5) It is shown that continuous phase transitions are possible between different the states of graviton-ghost condensate.

\section{Acknowledgements}

We are deeply grateful to Ludwig D. Faddeev of the Steklov Mathematical Institute and Mikhail Shifman and Arkady Vainshtein of the University of Minnesota for discussions of the structure and content of the theory. Also, we would like to express our deep appreciation to our friend and colleague Walter Sadowski for invaluable advise and help in the preparation of the manuscript.

\section{REFERENCES}

[1] L. Marochnik, D. Usikov and G. Vereshkov, Foundation of Physics, Vol. 38, 2008, pp. 546-555. doi:10.1007/s10701-008-9220-6

[2] M. H. Goroff and A. Sagnotti, Physics Letters B, Vol. 160, 1985, pp. 81-85.

[3] G. 't Hooft and M. Veltman, Annals of Institute Henri Poincare, Vol. 20, 1974, pp. 69-94.

[4] N. D. Birrell and P. C. W. Davies. "Quantum Fields in Curved Space,” Cambridge University Press, Cambridge, 1982. doi:10.1017/CBO9780511622632

[5] E. M. Lifshitz, Journal of Experimental and Theoretical Physics, Vol. 16, 1946, p. 587.

[6] L. P. Grishchuk, Soviet Physics-JETP, Vol. 40, 1975, pp. 409-415.

[7] R. A. Isaakson, Physical Review, Vol. 166, 1968, pp. 1263-1271. doi:10.1103/PhysRev.166.1263

[8] R. A. Isaakson, Physical Review, Vol. 166, 1968, pp. 1272-1280. doi:10.1103/PhysRev.166.1272

[9] L. S. Marochnik, N. V. Pelikhov and G. M. Vereshkov,
Astrophysics and Space Science, Vol. 34, 1975, pp. 249263. doi:10.1007/BF00644797

[10] L. S. Marochnik, N. V. Pelikhov and G. M. Vereshkov, Astrophysics and Space Science, Vol. 34, 1975, pp. 281294. doi:10.1007/BF00644799

[11] L. R. W. Abramo, R. H. Brandenberger and V. F. Mukhanov, Physical Review D, Vol. 56, 1997, pp. 3248-3257. doi:10.1103/PhysRevD.56.3248

[12] L. R. W. Abramo, Physical Review D, Vol. 60, 1999, Article ID: 064004. doi:10.1103/PhysRevD.60.064004

[13] V. Moncrief, Physical Review D, Vol. 18, 1978, pp. 983989. doi:10.1103/PhysRevD.18.983

[14] L. H. Ford and L. Parker, Physical Review D, Vol. 16, No. 6, 1977, pp. 1601-1608. doi:10.1103/PhysRevD.16.1601

[15] B. L. Hu and L. Parker, Physics Letters A, Vol. 63, 1977, pp. 217-220. doi:10.1016/0375-9601(77)90880-5

[16] Y. B. Zeldovich and A. Starobinsky, Letters to Journal of Experimental and Theoretical Physics, Vol. 26, 1977, pp. 252-255.

[17] B. S. DeWitt, “Quantum Gravity: A New Synthesis,” In: S. W. Hawking and W. Israel, Eds., General Relativity, Cambridge University Press, Cambridge, 1979, pp. 680743.

[18] S. W. Hawking, "The Path-Integral Approach to Quantum Gravity,” In S. W. Hawking and W. Israel, Eds., General Relativity, Cambridge University Press, Cambridge, 1979, pp. 746-785.

[19] G. Vereshkov and L. Marochnik, Journal of Modern Physics, Vol. 4, 2013, pp. 285-297. doi:10.4236/jmp.2013.42039

[20] L. D. Faddeev and A. A. Slavnov, "Gauge Fields. Introduction to Quantum Theory," 2nd Edition, Addison-Wesley Publishing Company, Boston, 1991.

[21] L. D. Faddeev and V. N. Popov, Physics Letters B, Vol. 25, 1967, pp. 29-30. doi:10.1016/0370-2693(67)90067-6

[22] B. S. DeWitt, Physical Review, Vol. 160, 1967, pp. 11131148. doi:10.1103/PhysRev.160.1113

[23] B. S. DeWitt, Physical Review, Vol. 162, 1967, pp. 11951239. doi:10.1103/PhysRev.162.1195

[24] L. Marochnik, D. Usikov and G. Vereshkov, “Graviton, Ghost and Instanton Condensation on Horizon Scale of the Universe. Dark Energy as Macroscopic Effect of Quantum Gravity,” 2008, pp. 1-93. ArXiv:0811.4484v2 [gr-qc]

[25] N. N. Bogolyubov, A. A. Logunov, A. I. Oksak and I. T. Todorov, "General Principles of Quantum Field Theory," Kluwer, Springer, 1990. doi:10.1007/978-94-009-0491-0

[26] L. D. Faddeev, "Hamilton Form of the Theory of Gravity," Proceedings of the V International Conference on Gravitation and Relativity, Tbilisi, 9-16 September 1968, pp. 54-56.

[27] L. D. Faddeev, Theoretical and Mathematical Physics, Vol. 1, 1969, pp. 1-13. doi:10.1007/BF01028566

[28] E. S. Fradkin and G. A. Vilkovisky, Physical Review D, Vol. 8, 1974, pp. 4241-4285.

[29] N. P. Konopleva, V. N. Popov and N. M. Queen, "Gauge 
Fields," Harwood Academic Publishers, Amsterdam, 1981.

[30] A. B. Borisov and V. I. Ogievetsky, Theoretical and Mathematical Physics, Vol. 21, 1975, pp. 1179-1188. doi:10.1007/BF01038096

[31] N. N. Bogolyubov and D. V. Shirkov, "The Theory of Quantum Fields,” Interscience, New York, 1959.

[32] P. J. Steinhardt and N. Turok, Physical Review D, Vol. 65, 2002, Article ID: 126003. doi:10.1103/PhysRevD.65.126003

[33] Y. B. Zeldovich, Letters to Journal of Experimental and Theoretical Physics, Vol. 6, 1967, pp. 316-317.

[34] S. Weinberg, Review of Modern Physics, Vol. 61, 1989, pp. 1-23. doi:10.1103/RevModPhys.61.1

[35] N. S. Kardashev, Astronomicheskii Zhurnal, Vol. 74, 1997, p. 803.

[36] R. Pasechnik, V. Beylin and G. Vereshkov, Journal of Cosmology and Astroparticle Physics, Vol. 1306, 2013, p. 11.

[37] R. Pasechnik, V. Beylin and G. Vereshkov, Physical Review D, Vol. 88, 2013, pp. 02359-023514.

[38] S. Nobbenhuis, "The Cosmological Constant Problem, an Inspiration for New Physics,” Ph.D. Thesis, Institute for Theoretical Physics Utrecht University, Leuvenlaan and Spinoza Institute, Utrecht, 2006.

[39] A. D. Dolgov, “The Very Early Universe," In: G. W. Gibbons, S. W. Hawking and S. T. C. Siklos, Eds., Proceedings of the Nuffield Workshop, Cambridge University Press, Cambridge, 1983, p. 449.

[40] L. H. Ford, Physical Review D, Vol. 35, 1987, pp. 23392344. doi:10.1103/PhysRevD.35.2339
[41] V. A. Rubakov, Physical Review D, Vol. 61, 2000, Article ID: 061501. doi:10.1103/PhysRevD.61.061501

[42] P. J. Steinhardt, N. Turok, Science, Vol. 312, 2006, pp. 1180-1183. doi:10.1126/science.1126231

[43] R. Rajaraman, "Solitons and Instantons. An Introduction to Solitons and Instantons in Quantum Field Theory," North-Holland Publishing Company, Amsterdam, 1982.

[44] A. Belavin and A. M. Polyakov, Letters to Jounal of Experimental and Theoretical Physics, Vol. 22, 1975, pp. 245-246.

[45] G. 't Hooft, Physical Review Letters, Vol. 37, 1976, pp. 8-11. doi:10.1103/PhysRevLett.37.8

[46] C. G. Callan Jr., R. F. Dashen and D. J. Gross, Physics Letters B, Vol. 63, 1976, pp. 334-340. doi:10.1016/0370-2693(76)90277-X

[47] R. Jackiw and C. Rebbi, Physical Review Letters, Vol. 36, 1976, pp. 1116-1119. doi:10.1103/PhysRevLett.36.1116

[48] R. Jackiw and C. Rebbi, Physical Review Letters, Vol. 37, 1976, pp. 1172-1175. doi:10.1103/PhysRevLett.37.172

[49] M. A. Shifman, "Instantons in Gauge Theories," World Scientific, Singapore, 1994.

[50] L. Marochnik, Gravitation and Cosmology, Vol. 19, 2013, pp. 178-187; arXiv:1204.4744 [gr-qc], 2012.

[51] V. A. Rubakov and P. G. Tinyakov, Physics Letters B, Vol. 214, 1988, pp. 334-338.

[52] V. A. Rubakov and P. G. Tinyakov, Letters to Journal of Experimental and Theoretical Physics, Vol. 48, 1988, pp. 327-330.

[53] V. A. Rubakov, Letters to Journal of Experimental and Theoretical Physics, Vol. 39, 1984, pp. 107-110. 


\section{Appendix}

\section{Renormalizations and Anomalies}

In Sections 10.1 and 10.2, we discuss a self-consistent theory of gravitons in isotropic Universe with the ghost sector not taken into account. As has been repeatedly stated, we believe that such a model is not mathematically sound. Gauges, completely removing the degeneracy, are absent in the theory of gravity. Thus, in the self-consistent theory of gravitons the ghost sector is inevitable present. Now, however, let us assume for the moment that the self-consistent theory of gravitons without ghosts is worth at least as a model of mathematical physics. The purpose of this Section is to get the properties of this model and to show that it is mathematically and physically internally inconsistent.

\subsection{Gravitons with No Ghosts. Vacuum Einstein Equations with Quantum Logarithmic Corrections}

It clear from the outset that in the non-ghost model the calculation of observables will be accompanied by the emergence of divergences. It is therefore necessary to formulate the theory in such a way that the regularization and renormalization operations are to be contained in its mathematical structure from the very beginning. We talk here about changes in the mathematical formulation of the theory. The relevant operations should be introduced into the theory with care: first, in the amended theory, coexistence of classical and quantum equations should be ensured automatically; second, the enhanced theory should not contain objects initially missing from the theory of gravity.

The dimensional regularization satisfies both abovementioned conditions. Important, however, is the following fact: the use of dimensional regularization suggests that the self-consistent theory of gravitons in the isotropic Universe is originally formulated in a spacetime of dimension $D=1+d$, where 1 is the dimension of time; $d=3-2 \varepsilon$ is the dimension of space. The special status of the time is due to the two factors: 1 ) all the events in the Universe, regardless of its actual dimension, are ordered along the one-dimensional temporal axis; 2) the canonical quantization of the graviton field in terms of the commutation relations for generalized coordinates and generalized momenta also presuppose the existence of the one-dimensional time. As for the space dimension, the limit transition to the true dimension $d=3$ is implemented after the regularization and renormalization.

Thus, we are working in a space with a metric

$$
\begin{aligned}
& d s^{2}=a^{2}(\eta)\left(d \eta^{2}-\gamma_{\alpha \beta} d x^{\alpha} d x^{\beta}\right), \quad \gamma^{\alpha \beta} \gamma_{\alpha \beta}=d, \\
& \sqrt{\left|g_{(d)}\right|}=a^{d+1}, R_{(d)}=-\frac{d}{a^{2}}\left(2 \frac{a^{\prime \prime}}{a}+(d-3) \frac{a^{\prime 2}}{a^{2}}\right) .
\end{aligned}
$$

To avoid mathematical contradictions that could arise at the limit $d \rightarrow 3$, Einstein equations in $D$-dimensional spacetime should be written down in exactly the form in which they were obtained from the variational principle:

$$
\begin{aligned}
& \frac{1}{\varkappa_{d}} \sqrt{\left|g_{(d)}\right|}\left(R_{0(d)}^{0}-\frac{1}{2} R_{(d)}\right) \\
& \equiv \frac{1}{2 \varkappa_{d}} d(d-1) a^{d-3} a^{\prime 2} \\
& =\frac{1}{8 \varkappa_{d}} a^{d-1} \sum_{k \sigma}\left\langle\Psi_{g}\left|\hat{\psi}_{k \sigma}^{\prime+} \hat{\psi}_{k \sigma}^{\prime}+k^{2} \hat{\psi}_{k \sigma}^{+} \hat{\psi}_{k \sigma}\right| \Psi_{g}\right\rangle, \\
& -\frac{d-1}{2 \varkappa_{d}} \sqrt{\left|g_{(d)}\right|} R_{(d)} \\
& \equiv \frac{1}{2 \varkappa_{d}} d(d-1)\left[2 a^{d-2} a^{\prime \prime}+(d-3) a^{d-3} a^{\prime 2}\right] \\
& =-\frac{d-1}{8 \varkappa_{d}} a^{d-1} \sum_{k \sigma}\left\langle\Psi_{g}\left|\hat{\psi}_{k \sigma}^{\prime+} \hat{\psi}_{k \sigma}^{\prime}-k^{2} \hat{\psi}_{k \sigma}^{+} \hat{\psi}_{k \sigma}\right| \Psi_{g}\right\rangle, \\
& \hat{\psi}_{k \sigma}^{\prime \prime}+(d-1) \frac{a^{\prime}}{a} \hat{\psi}_{k \sigma}^{\prime}+k^{2} \hat{\psi}_{k \sigma}=0 .
\end{aligned}
$$

Here $\varkappa_{d}$ is the Einstein gravitational constant in $D$ dimensional spacetime (Dimension $\left[\varkappa_{d} \hbar\right]=[l]^{D-2}$ ). The left hand sides of Equation (276) satisfy the Bianchi identity:

$$
\begin{aligned}
& \frac{1}{2 \varkappa_{d}} d(d-1)\left[a^{d-3} a^{\prime 2}\right] \\
& -\frac{1}{2 \varkappa_{d}} d(d-1) \frac{a^{\prime}}{a}\left[2 a^{d-2} a^{\prime \prime}+(d-3) a^{d-3} a^{\prime 2}\right] \equiv 0 .
\end{aligned}
$$

In the right hand side of Equation (276), the identity (278) generates condition of the graviton EMT conservation that satisfies if the equations of motion (277) are taken into account. Regarding the origin of the system of Equations (276) and (277), we should make the following comment. In this case it is inappropriate to invoke the reference to the path integral and factorization of its measures because the path integral inevitably leads to the theory of ghosts interacting with the macroscopic gravity. We can only mention a heuristic recipe: one should refer to the density of Einstein equations with mixed indices, define the exponential parameterization of the metric, and expand the equations into a series of metric fluctuations with an accuracy of the second-order terms. Deviations from this recipe (for example, linear parameterization $\hat{g}_{i k}=g_{i k}+\hat{h}_{i k}$ ) lead to a system of inconsistent classical and quantum equations. To remove this sort of inconsistency, one is forced to use artificial transactions outside the formalism of the theory (see, for example, [11]).

While working with the system of Equations (276) and (277), we face with two mathematical problems. The first 
problem is that in the framework of that system of equations, except in very special cases, it is impossible to formulate the dynamics of operators on a given background that is to get the solution of the Equation (277) as an accurate operator function of time. This is due to the fact that formulae of (276) in reality are not yet specific equations. They are only a layout of Einstein equations with radiation corrections. These equations can only be obtained after regularization and renormalizations of the ultraviolet divergences. In addition, the functional form of equations depends on which quantum gravitational effects are to be taken into account outside the sector of vacuum (i.e. zero) fluctuations of the graviton field. The only possible way to study the system of Equations (276) and (277) is 1) to obtain the solution of operator equation (277) in a form of a functional of the scale factor without specifying the dependence on $a(\eta)$ with a clear emphasis on zero fluctuations in this functional; 2) to substitute the obtained functional in (276) under certain assumptions about the state vector; 3) to regularize and renormalize and finally 4) to solve the macroscopic Einstein equations, obtained after these operations. Implementation of the program, an essential element of which is the allocation of zero fluctuations generating ultraviolet divergences, is possible only when using the method of asymptotic expansions of solutions of operator equation in the square of wavelength of the graviton modes. Thus, the problem of the lack of macroscopic Einstein equations in the original formulation of this theory with divergences limits the methods of this theory to the short-wave approach. Note that this fact was clearly indicated by DeWitt [17].

The second problem is related to the infrared instability of the theory, with the object of the theory being a conformal non-invariant massless quantum field. The problem is due to the fact that not every representation of the asymptotic series can be substituted into energymomentum tensor to perform the summation over the wave numbers. For example, if in the explicit form, a term in the asymptotic series contains a large parameter $k^{2 n}$ in the denominator, then starting from $n=2$ in the integration over the wave numbers the infrared divergences will appear. Such an asymptotic series can not be used even for the renormalization of ultraviolet divergences, because when it is used in the space of the physical dimension $d=3$, the logarithmic divergences arise simultaneously at the ultraviolet and the infrared limits. In the method of dimensional regularization the problem is reduced to the fact that it is impossible to choose an interim dimension $d$ in a way such that the integral exists at both limits.

Formally, the technical problem described above is partly solved by reformatting the asymptotic series. In particular, the following method will be used, in which parameter of the asymptotic expansion is the effective frequency

$$
\begin{aligned}
& \omega_{k}^{2}=k^{2}+\rho, \\
& \rho=\frac{d-1}{4 d} a^{2} R_{(d)}=-\frac{d-1}{4}\left[2 \frac{a^{\prime \prime}}{a}+(d-3) \frac{a^{\prime 2}}{a^{2}}\right] .
\end{aligned}
$$

In this method, the integrals over the wave numbers can be defined in terms of the principal value. Contributions of the poles at $k=\sqrt{-\rho}$ can not be mathematically verified if only because there are such contributions from each term of the infinite asymptotic series. The inability to describe infrared effects is the principal disadvantage of a theory with divergences, which uses only asymptotic expansions with respect to the wavelength. Meanwhile, as general considerations and the results of this work show, in the physics of conformal non-invariant massless field the most interesting and innovative effects occur in the infrared spectrum. The method of describing these effects, based on the exact BBGKY chain, can not be used in the theory with divergences, because a method regularizing the infinite chain of moments of the spectral function does not exist.

The above problems automatically reduces the interest toward the theory with divergences. However, given that all previous works in this area have been implemented in the framework of regularization and renormalization, let us conduct our analysis to the end. In calculations, it is enough to consider the equation for the convolution. After identity transformations, using the equation of motion (277), we get

$$
\begin{aligned}
& \frac{1}{2 \varkappa_{d}} d(d-1)\left[2 a^{d-2} a^{\prime \prime}+(d-3) a^{d-3} a^{\prime 2}\right] \\
& =-\frac{d-1}{16 \varkappa_{d}} \sum_{k \sigma}\left(W_{k \sigma}^{\prime} a^{d-1}\right),
\end{aligned}
$$

where

$$
W_{k \sigma}=\left\langle\Psi_{g}\left|\hat{\psi}_{k \sigma}^{+} \hat{\psi}_{k \sigma}\right| \Psi_{g}\right\rangle
$$

is the spectral function of gravitons. The calculation of the spectral function by the method of asymptotic expansion with respect to the square of wavelength was described in Section 4.1. Now we need to repeat this calculation excluding the ghosts, but with input from zero fluctuations in the spacetime of dimension $D=d+1$. The relevant calculations do not require additional comments. A spectral function is represented as:

$$
W_{k \sigma}=W_{k \sigma}^{(v a c)}+W_{k \sigma}^{(e x c)},
$$

where $W_{k \sigma}^{(v a c)}$ is the vacuum component of the spectral function and $W_{k \sigma}^{(e x c)}$ is the spectral function of excitations. After passage to the limit $d \rightarrow 3$, the contribution of $W_{k \sigma}^{(e x c)}$ to the EMT of short gravitons is exactly the 
same as (129), (130). In the future, we discuss only the contribution from vacuum components of the spectral function. In the calculations, we must keep in mind that in the $d$-dimensional space the number of internal degrees of freedom of transverse gravitons is

$w_{g}=(d+1)(d-2) / 2$. The solution for the vacuum spectral function is expressed in terms of the functional (124):

$$
\begin{aligned}
& \sum_{\sigma} W_{k \sigma}^{(v a c)} \\
& =\frac{4 \varkappa_{d} \hbar}{a^{d-1}} \cdot \frac{(d+1)(d-2)}{4 \varepsilon_{k}} \\
& =\frac{4 \varkappa_{d} \hbar}{a^{d-1}} \cdot \frac{(d+1)(d-2)}{4 \omega_{k}} \sum_{s=0}^{\infty}(-1)^{s} \hat{J}_{k}^{s} \cdot 1 .
\end{aligned}
$$

The powers of operator $\hat{J}_{k}^{s} \cdot 1$ are defined by formulas (125), in which $\omega_{k}^{2}$ has the form (279). After substitution of (282) into (280), the zero-term in the asymptotic expansion creates an integral, calculated by the rules of dimensional regularization:

$$
\begin{aligned}
& \sum_{k} \frac{1}{\omega_{k}} \\
& =\frac{1}{(2 \pi)^{d}} \frac{2 \pi^{d / 2}}{\Gamma(d / 2)} \int_{0}^{\infty} \frac{k^{d-1} \mathrm{~d} k}{\left(k^{2}+\rho\right)^{1 / 2}} \\
& =\frac{\Gamma[(3-d) / 2]}{2^{d-1} \pi^{(d+1) / 2}(1-d)} \rho^{(d-1) / 2} .
\end{aligned}
$$

The $\Gamma$-function in (283) diverges for $d \rightarrow 3$. Therefore, calculation of the integral (283) and transformation of expressions with $\Gamma$-functions are carried out with those values of $d$ which provide the existence of the integral and $\Gamma$-functions. At the final stage, the result of these calculations is analytically continued to the vicinity $d=3$. All other terms of the asymptotic expansion (282) generate finite integrals and do not require a dimensional regularization. For reasons of heuristic rather than mathematical nature, it is considered that these terms are negligible compared to the contribution of the principal term of the asymptotic expansion (see below the effective Lagrangian (293)). Convolution of $D$-dimensional Einstein's Equation (280), containing the main term of the vacuum EMT of gravitons, has the form:

$$
\begin{aligned}
& \frac{1}{2 \varkappa_{d}} d(d-1)\left[2 a^{d-2} a^{\prime \prime}+(d-3) a^{d-3} a^{\prime 2}\right] \\
& =\frac{\hbar(d+1)(d-2)}{2^{d+3} \pi^{(d+1) / 2}} \Gamma\left(\frac{3-d}{2}\right)\left[\left(\frac{\rho^{(d-1) / 2}}{a^{d-1}}\right) a^{d-1}\right] .
\end{aligned}
$$

Other Einstein equations can be obtained using the Bianchi identities. A complete system of Einstein vacuum equations is written in $D$-covariant form:

$$
\begin{aligned}
& R_{i(d)}^{k}-\frac{1}{2} \delta_{i}^{k} R_{(d)} \\
& +\frac{\varkappa_{d} \hbar(d+1)(d-2)(d-1)^{\frac{d-1}{2}}}{2^{2 d+2}(d \pi)^{\frac{d+1}{2}}} \Gamma\left(\frac{3-d}{2}\right) \\
& \times\left[\left(R_{(d)}^{\frac{d-1}{2}}\right)_{; i}^{; k}-\delta_{i}^{k}\left(R_{(d)}^{\frac{d-1}{2}}\right)_{; l}^{; l}-\left(R_{i(d)}^{k}-\frac{1}{d+1} \delta_{i}^{k} R_{(d)}\right) R_{(d)}^{\frac{d-1}{2}}\right] \\
& =0 .
\end{aligned}
$$

Equation (285) are obtained by the variation of action

$$
\begin{aligned}
& S_{v a c}=\int \sqrt{\left|g_{(d)}\right|} \mathrm{d}^{D} x \\
& {\left[-\frac{1}{2 \varkappa_{d}} R_{(d)}+\frac{\hbar(d-2)(d-1)^{\frac{d-1}{2}}}{2^{2 d+2}(d \pi)^{\frac{d+1}{2}}} \Gamma\left(\frac{3-d}{2}\right) R_{(d)}^{\frac{d+1}{2}}\right] .}
\end{aligned}
$$

It is obvious from (285), (286) that the method of dimensional regularization retains overall covariance of the theory. Of course, quantum corrections, appearing in (285), satisfy the condition of conservation.

Renormalization and removal of regularization (limit $d \rightarrow 3$ ) are held at the level of action. A parameter with the dimension of length, which will eventually acquire the status of renormalization scale, is contained within the theory. This parameter, referred to as $L_{g}$, is appears in the $D$-dimensional constant of gravity:

$$
\varkappa_{d}=\varkappa \cdot L_{g}^{d-3} .
$$

The technique of removal the regularization assumes conservation of dimensionality for those objects in which the limit operation is performed. There are two such objects: the measure of integration $d \mu$ and the density of the Lagrangian $\mathcal{L}$. As can be seen from (286), (287), the first (Einstein) term of the action is written down as

$$
\begin{aligned}
& S_{\text {vac }}^{(1)}=\int \mathcal{L}^{(1)} \mathrm{d} \mu, \quad \mathcal{L}^{(1)}=-\frac{1}{2 \varkappa} R_{(d)}, \\
& d \mu=\sqrt{\left|g_{(d)}\right|} L_{g}^{4-D} d^{D} x,
\end{aligned}
$$

where $D$-dimensional objects $\mathcal{L}$ and $d \mu$ have the same dimensions as the corresponding 4-dimensional objects. In this sector of the theory the limit transition is trivial: $R_{(d)} \rightarrow R, \quad d \mu \rightarrow \sqrt{-g} d^{4} x$. In the sector of quantum corrections to the Einstein theory, we introduce the same measure and obtain the density of the Lagrangian:

$$
\mathcal{L}^{(2)}=\frac{\hbar L_{g}^{d-3}(d-2)(d-1)^{\frac{d-1}{2}}}{2^{2 d+2}(d \pi)^{\frac{d+1}{2}}} \Gamma\left(\frac{3-d}{2}\right) R_{(d)}^{\frac{d+1}{2}}
$$


It is necessary to emphasize that the operations of renormalizations and removal of regularization have to be mathematically well-defined and generally-covariant. The condition of mathematical certainty assumes that the renormalization is conducted before the lifting of regularization. At the same time, the general-covariance of the procedure is automatically fulfilled if the counterterms imposed in the Lagrangian are the $D$-dimensional invariants. Note also that if the mathematical value is finite at $d=3$, then the above formulated conditions do not prevent the expansion of this quantity in a Taylor series over the parameter $(3-d) / 2$. In particular, we can write:

$$
\begin{aligned}
L_{g}^{d-3} R_{(d)}^{\frac{d+1}{2}} & \equiv R_{(d)}^{2}\left(L_{g}^{2} R_{(d)}\right)^{\frac{d-3}{2}} \\
& =R_{(d)}^{2}\left(1+\frac{3-d}{2} \ln \frac{\mu_{g}^{2}}{R_{(d)}}+\cdots\right),
\end{aligned}
$$

where $\mu_{g}=1 / L_{g}$; ellipsis designate the terms which do not contribute to the final result. The substitution (290) in (289) provides:

$$
\begin{aligned}
\mathcal{L}^{(2)} & =\frac{\hbar(d-2)(d-1)^{\frac{d-1}{2}}}{2^{2 d+2}(d \pi)^{\frac{d+1}{2}}} \Gamma\left(\frac{3-d}{2}\right) R_{(d)}^{2} \\
& =\frac{\hbar(d-2)(d-1)^{\frac{d-1}{2}}}{2^{2 d+2}(d \pi)^{\frac{d+1}{2}}} \Gamma\left(\frac{5-d}{2}\right) R_{(d)}^{2} \ln \frac{\mu_{g}^{2}}{R_{(d)}}+\cdots
\end{aligned}
$$

According to (291), the source Lagrangian of the theory requires a $D$-invariant counter-term, which removes the contribution proportional to the diverging $\Gamma$-function:

$$
\begin{aligned}
\mathcal{L}_{0}^{(2)}= & -\frac{\hbar(d-2)(d-1)^{\frac{d-1}{2}}}{2^{2 d+2}(d \pi)^{\frac{d+1}{2}}} \Gamma\left(\frac{3-d}{2}\right) R_{(d)}^{2} \\
& +\frac{\hbar}{4 f^{2}} R_{(d)}^{2} .
\end{aligned}
$$

In (292), there is a new finite constant of the theory of gravity $1 / f^{2}$. The removal of the regularization in the renormalized Lagrangian is conducted by the regular transition:

$$
\begin{aligned}
\mathcal{L}_{\text {ren }} & =\lim _{d \rightarrow 3}\left(\mathcal{L}^{(1)}+\mathcal{L}^{(2)}+\mathcal{L}_{0}^{(2)}\right) \\
& =-\frac{1}{2 \varkappa} R+\frac{\hbar}{4 f^{2}} R^{2}+\frac{\hbar}{1152 \pi^{2}} R^{2} \ln \frac{\mu_{g}^{2}}{R} \\
& =-\frac{1}{2 \varkappa} R+\frac{\hbar}{1152 \pi^{2}} R^{2} \ln \frac{\lambda_{g}^{2}}{R},
\end{aligned}
$$

where

$$
\lambda_{g}^{2}=\mu_{g}^{2} \exp \frac{288 \pi^{2}}{f^{2}}
$$

is the renorm-invariant scale. There is a heuristic argument allowing to use the obtained expression: quantum corrections in the Lagrangian (ref (12.19)) dominate over all other neglected terms of the asymptotic series over The logarithmic parameter $\ln \left(\lambda_{g}^{2} / R\right) \gg 1$.

The renormalized Einstein vacuum equations with quantum corrections obtained from the Lagrangian (293) are as follows:

$$
\begin{aligned}
& R_{i}^{k}-\frac{1}{2} \delta_{i}^{k} R \\
&+\frac{2 \hbar \hbar}{288 \pi^{2}}\left\{\left[R \ln \frac{\lambda_{g}^{2}}{R}\right]_{; i}^{; k}-\delta_{i}^{k}\left[R \ln \frac{\lambda_{g}^{2}}{R}\right]_{; l}^{; l}\right. \\
&\left.-\left(R R_{i}^{k}-\frac{1}{4} \delta_{i}^{k} R^{2}\right) \ln \frac{\lambda_{g}^{2}}{R}-\frac{1}{8} \delta_{i}^{k} R^{2}\right\}=0 .
\end{aligned}
$$

Note that exactly the same equations are obtained from $D$-dimensional Equation (285), provided that the operations are performed in the same sequence: first $a$ renormalization with the introduction of D-covariant counter-terms is conducted, and then a limit transition to the physical dimension is performed.

\subsection{Intrinsic Contradiction of Theory with No Ghosts: Impossibility of One-Loop Renormalization}

We are still discussing a formal model—self-consistent theory of gravitons with no ghosts. In the previous section it was shown that the renormalization of divergences, that inevitably arise in this model, requires the imposition of an additional term quadratic in the curvature in the Lagrangian. It is now necessary to draw attention to two mathematical facts: 1) the need for a modification of Einstein theory is caused by quantum effects contained in the Lifshitz operator Equation (277); 2) the original Lagrangian and operator equations of the modified theory have the form:

$$
\begin{aligned}
& \mathcal{L}= \int\left(-\frac{1}{2 \varkappa} \hat{R}+\frac{\hbar}{4 f^{2}} \hat{R}^{2}\right) \sqrt{-\hat{g}} \mathrm{~d}^{4} x \\
& \sqrt{-\hat{g}}\left[\frac{1}{\varkappa}\left(\hat{R}_{i}^{k}-\frac{1}{2} \delta_{i}^{k} \hat{R}\right)\right. \\
&\left.\quad+\frac{\hbar}{f^{2}}\left(\hat{D}_{i} \hat{D}^{k} \hat{R}-\delta_{i}^{k} \hat{D}_{l} \hat{D}^{l} \hat{R}-\hat{R} \hat{R}_{i}^{k}+\frac{1}{4} \delta_{i}^{k} \hat{R}^{2}\right)\right] \\
&=0,
\end{aligned}
$$

where $\hat{D}_{i}$ is a covariant derivative in a space with the operator metric $\hat{g}_{i k}$. It is quite obvious that these facts 
contradict each other: the quantum effects in the Lifshitz equation lead to a theoretical model that contradicts the Lifshitz equation. Let us demonstrate that the contradiction is a direct consequence of the non-renormalizability of the model (295) off the graviton mass shell.

Equation (296), after their linearization describe quantized waves of two types - tensor and scalar. It makes sense to discuss the problem of the scalar modes only in the event that at least preliminary criteria for consistency of modified theory will be obtained. Therefore, first of all, we should reveal properties of the tensor modes. Here is an expression for the Lagrangian of a system consisting of self-consistent cosmological field and tensor gravitons:

$$
\begin{aligned}
S= & \int \mathrm{d} t N a^{3}\left\{-\frac{3}{\varkappa N^{2}} \frac{\dot{a}^{2}}{a^{2}}+\frac{9 \hbar}{f^{2} N^{4}}\left(\frac{\ddot{a}}{a}-\frac{\dot{N}}{N} \frac{\dot{a}}{a}+\frac{\dot{a}^{2}}{a^{2}}\right)^{2}\right. \\
& +\frac{1}{8}\left[\frac{1}{\varkappa}+\frac{6 \hbar}{N^{2} f^{2}}\left(\frac{\ddot{a}}{a}-\frac{\dot{N}}{N} \frac{\dot{a}}{a}+\frac{\dot{a}^{2}}{a^{2}}\right)\right] \\
& \left.\times \sum_{k \sigma}\left(\frac{1}{N^{2}} \frac{\mathrm{d} \hat{\psi}_{k \sigma}^{+}}{\mathrm{d} t} \frac{\mathrm{d} \hat{\psi}_{k \sigma}}{\mathrm{d} t}-\frac{k^{2}}{a^{2}} \hat{\psi}_{k \sigma}^{+} \hat{\psi}_{k \sigma}\right)\right\} .
\end{aligned}
$$

The equation for gravitons is produced either by the linearization of the Equation (296), or from (297) by the variation procedure:

$$
\begin{aligned}
& \left(1-\frac{2 \hbar}{f^{2}} R\right)\left(\hat{\psi}_{k \sigma}^{\prime \prime}+2 \frac{a^{\prime}}{a} \hat{\psi}_{k \sigma}^{\prime}+k^{2} \hat{\psi}_{k \sigma}\right) \\
& -\frac{2 \hbar}{f^{2}} R^{\prime} \hat{\psi}_{k \sigma}^{\prime}=0 .
\end{aligned}
$$

Please note that the last term in (298) makes it impossible to retain the Lifshitz equation. After the transformation

$$
\hat{\psi}_{k \sigma}=a^{-1}\left(1-\varkappa \hbar R / f^{2}\right)^{-1 / 2} \hat{\varphi}_{k \sigma}
$$

Equation (298) has a form

$$
\hat{\varphi}_{k \sigma}^{\prime \prime}+\left[k^{2}+a^{2}\left(\frac{R}{6}+P\right)\right] \hat{\varphi}_{k \sigma}=0 .
$$

In (299), the deviation from the Lifshitz equation is manifested in the effective frequency of gravitons - the latter contains an additional function of curvature's derivatives

$$
\begin{aligned}
P & =-\frac{1}{2}\left[\ln \left(1-\varkappa \hbar R / f^{2}\right)\right]_{; l}^{; l}-\frac{1}{4} \\
& -\left[\ln \left(1-\varkappa \hbar R / f^{2}\right)\right]^{j l}\left[\ln \left(1-\varkappa \hbar R / f^{2}\right)\right]_{; l} .
\end{aligned}
$$

When calculating quantum corrections to the macroscopic equations, the modification of the effective frequency leads to additional divergences. Averaged vacu- um Equation (296), after their polynomial expansion in powers of curvature, look as follows (finite logarithmic corrections are omitted):

$$
\begin{aligned}
& R_{i}^{k}-\frac{1}{2} \delta_{i}^{k} R \\
& +\varkappa \hbar\left(\frac{\Gamma(\varepsilon)}{288 \pi^{2}}+\frac{1}{f_{0}^{2}}\right)\left(R_{; i}^{; k}-\delta_{i}^{k} R_{; l}^{; l}-R R_{i}^{k}+\frac{1}{4} \delta_{i}^{k} R^{2}\right) \\
& +(\varkappa \hbar)^{2} \frac{\Gamma(\varepsilon)}{48 \pi^{2} f_{0}^{2}} \\
& \cdot\left(R_{; l ; i}^{; l ; k}-\delta_{i}^{k} R_{; l ; m}^{; l ; m}-R_{i}^{k} R_{; l}^{; l}+\frac{1}{2} R_{; i} R^{; k}-\frac{1}{4} \delta_{i}^{k} R_{; l} R^{; l}\right)=0 .
\end{aligned}
$$

Here $\Gamma(\varepsilon) \sim 1 / \varepsilon$ is a divergent $\Gamma$-function obtained by dimensional regularization; $1 / f_{0}^{2}$ is a seed constant of a theory with quadratic invariant. The complete quantum Lagrangian corresponding to Equation (301) has the form:

$$
\begin{aligned}
\mathcal{L}=\int & {\left[-\frac{1}{2 \varkappa} \hat{R}+\hbar\left(\frac{\Gamma(\varepsilon)}{1152 \pi^{2}}+\frac{1}{4 f_{0}^{2}}\right) \hat{R}^{2}\right.} \\
& \left.+\varkappa \hbar^{2} \frac{\Gamma(\varepsilon)}{192 \pi^{2} f_{0}^{2}} \hat{R}^{\prime l} \hat{R}_{; l}\right] \sqrt{-\hat{g}} \mathrm{~d}^{4} x .
\end{aligned}
$$

Renormalization of the second term in (302) is performed by selecting the seed constant:

$$
\frac{1}{f_{0}^{2}}=-\frac{\Gamma(\varepsilon)}{288 \pi^{2}}+\frac{1}{f^{2}} .
$$

However, a divergent coefficient forms before the third term. To overcome this divergence, it is necessary to introduce a new seeding "fundamental" constant of the modified theory of gravity $1 / h_{0}^{2}$ with a renormalization rule:

$$
\frac{1}{h_{0}^{2}}=\frac{\Gamma(\varepsilon)}{48 \pi^{2}}\left(\frac{\Gamma(\varepsilon)}{288 \pi^{2}}-\frac{1}{f^{2}}\right)+\frac{1}{h^{2}} .
$$

Further actions are obvious and pointless: Lifshitz equation is the subject of the next modification; quantum corrections generate another new divergence; to renormalize the new divergence a new theory of gravity is introduced, etc. The only conclusion to be drawn from this procedure is that based on the criteria of quantum field theory, the one-loop self-consistent theory of gravitons in the isotropic Universe, and not possessing the property of one-loop finiteness outside of mass shell, does not exists as a mathematical model. In such a theory it is impossible to quantitatively analyze any physical effect. The theory of gravitons without ghosts is nonrenormalizable even in the one-loop approximation. It is also important to stress that the correct alternative to a 
non-renormalizable theory is only a finite theory with the graviton-ghost compensation of divergences.

In the future, from our perspective, the method of regularization and renormalization in general will be excluded from the arsenal of quantum theory of gravity, including one from the theory of one-loop quantum effects involving matter fields. Correct alternatives to existing methods of analysis of these effects to be found in extended supergravities, finite at least in one-loop approximation.

The situation prevailing in the scientific literature is a paradoxical one. On the one hand, inadequate nature of the regularization and renormalization methods in the quantum theory of gravity should be obvious from the latest development trends in the theories of supergravity and superstrings. On the other hand, however, in all works we know on cosmological applications of oneloop quantum gravity theoretical models are used, which, according to the criteria of quantum field theory, do not exist. We cannot comment on the specific results obtained in these models by the reasons clear from the content of this section. Once again we should emphasize that the self-consistent theory of gravitons, if it exists as a theoretical model, must be finite outside the mass shell of gravitons. Effects arising in the finite theory are described in the main text of this work. 